\title{
Selectivity Control in Gold-Catalyzed Hydroarylation of Alkynes with Indoles: Application to Unsymmetrical Bis(indolyl)methanes
}

Euan B. McLean, Francesca M. Cutolo, Orla J. Cassidy, David J. Burns and Ai-Lan Lee*

\section{SUPPORTING INFORMATION}

\section{Contents}

1- General Information S-2

2- Optimisation Studies $\quad$ S-3

3- Synthesis of Bis(indolyl)methane Products- Initial Studies S-5

$\begin{array}{lr}\text { 4- Starting Material Synthesis } & \text { S-7 }\end{array}$

5- General Procedures for the Gold(I)-Catalysed Hydroarylation of Alkynes S-9

$\begin{array}{lr}\text { 6- Product Characterisation } & \text { S-10 }\end{array}$

$\begin{array}{lr}\text { 7- NMR Spectra } & \text { S-29 }\end{array}$

8- References $\quad$ S-59 


\section{General Information}

${ }^{1} \mathrm{H}$ NMR spectra were recorded on a Bruker AV300 or AV400 spectrometer at $300 \mathrm{MHz}$ or 400 $\mathrm{MHz}$ respectively and referenced to residual solvent. ${ }^{13} \mathrm{C}$ NMR spectra were recorded using the same spectrometers at $75 \mathrm{MHz}$ or $101 \mathrm{MHz}$ respectively. Chemical shifts ( $\delta$ in ppm) were referenced to tetramethylsilane (TMS) or to residual solvent peaks $\left(\mathrm{CDCl}_{3}\right.$ at $\delta_{\mathrm{H}} 7.26 \mathrm{ppm}$ and $\delta_{\mathrm{c}}$ at $\left.77.0 \mathrm{ppm}\right) .{ }^{19} \mathrm{~F}$ NMR were recorded using the same spectrometers at $282 \mathrm{MHz}$ or 376 $\mathrm{MHz}$ respectively. $J$ values are given in $\mathrm{Hz}$ and $\mathrm{s}, \mathrm{d}, \mathrm{t}, \mathrm{q}, \mathrm{dd}, \mathrm{ddd}, \mathrm{m}$ and $\mathrm{dm}$ are abbreviations corresponding to singlet, doublet, triplet, quartet, doublet of doublets, doublet of doublets of doublets, multiplet and doublet of multiplets respectively. Mass spectra were obtained at the EPSRC UK National Mass Spectrometry Facility at Swansea University or at Syngenta. Infrared spectra were obtained on Perkin-Elmer Spectrum 100 FT-IR Universal ATR Sampling Accessory, deposited neat or as a chloroform solution to a diamond/ZnSe plate.

Column chromatography was carried out using Matrix silica gel 60 from Fluorochem and TLC performed using Merck silica gel $60 \mathrm{~F}_{254}$ pre-coated sheets and visualised by UV $(254 \mathrm{~nm})$ and/or aqueous acidic $\mathrm{KMnO}_{4}$. Anhydrous solvents were obtained from a MBRAUN SPS-800 solvent purification system (SPS) and stored under an argon atmosphere until use. $\mathrm{PPh}_{3} \mathrm{AuNTf}_{2}$ (2:1 toluene adduct) was purchased from Sigma-Aldrich. Where heating of reaction was required, the reactions were heated through use of an aluminium heating block of appropriate size on an IKA hotplate/stirrer set to heat at the given temperature. 


\section{Optimisation Studies}

Optimisation initially commenced utilising both substrates $\mathbf{1 a}$ and $\mathbf{1 p}$ and initial solvent screening was carried out using indole $\mathbf{1 p}$.

Table S-1: Initial Solvent Screen<smiles>Cn1ccc2ccccc21</smiles>

1 equiv.

$$
+\quad \mid
$$

$2 a$

1 equiv.

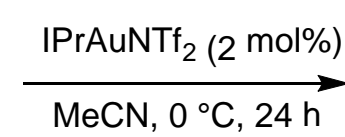

$\mathrm{MeCN}, 0{ }^{\circ} \mathrm{C}, 24 \mathrm{~h}$

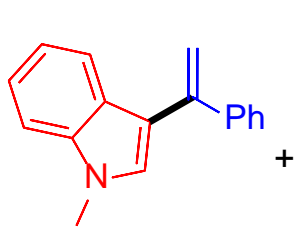

$4 p$

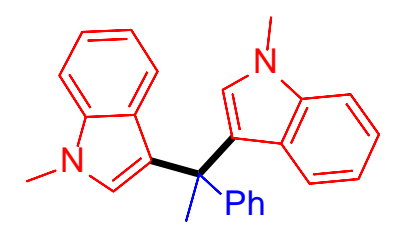

$3 p$

\begin{tabular}{|c|c|c|c|c|}
\hline Entry & Solvent & $1 p(\%)^{a}$ & $4 p(\%)^{a}$ & $3 p(\%)^{a}$ \\
\hline 1 & MeCN & $0 \%$ & Trace & $96 \%$ \\
\hline 2 & DMF & $100 \%$ & $0 \%$ & $0 \%$ \\
\hline 3 & Toluene & $20 \%$ & $0 \%$ & $52 \%$ \\
\hline 4 & DCM & $0 \%$ & $0 \%$ & $100 \%$ \\
\hline
\end{tabular}

aield determined by ${ }^{1} \mathrm{H}$ NMR analysis using 2,6-dimethoxy-1 4-benzoquinone as an internal standard. $\operatorname{IPr}=$ 1,3-bis(2,6-diisopropylphenyl)imidazol-2-ylidene.

From this initial solvent screen, it was determined that acetonitrile was the most promising solvent as it was the only solvent in which any trace of desired vinylindole $4 p$ could be detected. At this initial point of the optimisation study, other experiments carried out in parallel identified substrate 1a as a more promising substrate as it yielded more promising results. Therefore, acetonitrile was taken forward as the solvent choice for further optimisation studies utilising indole 1a as the model substrate. 
Table S-2: Further Optimisation<smiles>C#C[PH2+]c1cc2ccccc2[nH]1</smiles>
$x$ equiv. $\quad y$ equiv.

\begin{tabular}{llllllll} 
Entry & $\begin{array}{l}\text { Temp } \\
\left({ }^{\circ} \mathrm{C}\right)\end{array}$ & $\begin{array}{l}\mathrm{L} \\
(\mathrm{z} \mathrm{mol} \%)\end{array}$ & $\mathrm{x}$ & $\mathrm{y}$ & $\mathbf{1 a}(\%)^{\mathrm{a}}$ & $\mathbf{4 a}(\%)^{\mathrm{a}}$ & $\mathbf{3 a}(\%)^{\mathrm{a}}$ \\
\hline 1 & 0 & $\operatorname{IPr}(2)$ & 1 & 1 & $76 \%$ & $24 \%$ & $0 \%$ \\
2 & 0 & $\operatorname{Pr}(2)$ & 3 & 1 & $\mathrm{n} / \mathrm{a}^{\mathrm{b}}$ & $47 \%$ & $28 \%$ \\
3 & 0 & $\operatorname{Pr}(2)$ & 5 & 1 & $\mathrm{n} / \mathrm{a}^{\mathrm{b}}$ & $45 \%$ & $26 \%$ \\
4 & 0 & $\mathrm{PPh}_{3}(2)$ & 1 & 1 & $77 \%$ & $23 \%$ & $0 \%$ \\
5 & 0 & $\mathrm{PPh}_{3}(2)$ & 3 & 1 & $\mathrm{n} / \mathrm{a}^{\mathrm{b}}$ & $46 \%$ & $10 \%$ \\
$6^{\mathrm{c}}$ & 0 & $\mathrm{PPh}_{3}(2)$ & 3 & 1 & $\mathrm{n} / \mathrm{a}^{\mathrm{b}}$ & $55 \%$ & $8 \%$ \\
7 & 0 & $\mathrm{PPh}_{3}(4)$ & 3 & 1 & $\mathrm{n} / \mathrm{a}^{\mathrm{b}}$ & $49 \%$ & $20 \%$ \\
8 & 0 & $\mathrm{PPh}_{3}(2)$ & 5 & 1 & $\mathrm{n} / \mathrm{a}^{\mathrm{b}}$ & $18 \%$ & $55 \%$ \\
9 & 0 & $\mathrm{PPh}_{3}(2)$ & 1 & 4 & $70 \%$ & $30 \%$ & $0 \%$ \\
10 & 0 & $\mathrm{PPh}_{3}(2)$ & 1 & 5 & $69 \%$ & $31 \%$ & $0 \%$ \\
11 & 0 & $\mathrm{PPh}_{3}(2)$ & 1 & 3 & $55 \%$ & $45 \%$ & $0 \%$ \\
$12^{\mathrm{c}}$ & 0 & $\mathrm{PPh}_{3}(2)$ & 1 & 3 & $44 \%$ & $56 \%$ & $0 \%$ \\
13 & 30 & $\mathrm{PPh}_{3}(2)$ & $\mathbf{1}$ & $\mathbf{3}$ & $\mathbf{0 \%}$ & Quant. & $\mathbf{0 \%}$ \\
14 & 30 & $\mathrm{PPh}_{3}(2)$ & 1 & 1 & $10 \%$ & $90 \%$ & $0 \%$ \\
\hline
\end{tabular}

${ }^{a}$ Yield determined by ${ }^{1} \mathrm{H}$ NMR analysis using 2,6-dimethoxy-1 4-benzoquinone as an internal standard. ${ }^{b}$ Not applicable as $1 \mathrm{a}$ is in excess. 'Reaction time of $48 \mathrm{~h}$. IPr = 1,3-bis(2,6-diisopropylphenyl)imidazol2-ylidene.

In summary for the optimisation stage, it would seem that it is a combination of the solvent, catalyst and stoichiometry which allows for a switch in the selectivity of the hydroarylation reaction; rather than there being one specific factor which results in desired vinylindole 4 a being produced selectively. 


\section{Synthesis of Bis(indolyl)methanes - Initial Studies}

Investigations into the synthesis of unsymmetrical BIMs 5 commenced by optimising the acidcatalysed addition of the second indole separately, choosing vinyl indole $\mathbf{4 f}$ and indole $\mathbf{1 b}$ as the model substrates (Table S-2). Unfortunately, desired bis(indolyl)methane $\mathbf{5 d}$ could not be observed under any of the conditions investigated. Instead, displacement of the indole substituent already present in substrate $\mathbf{4 f}$ occurred to form symmetrical bis(indolyl)methane $\mathbf{3 b}$.

Table S-3: Initial Optimisation of Bis(indolyl)methane Synthesis<smiles>C=C(c1ccccc1)c1c(-c2ccccc2)[nH]c2ccccc12</smiles>
$4 \mathbf{f}$<smiles>[X]C([X])([O-])[Na]</smiles>

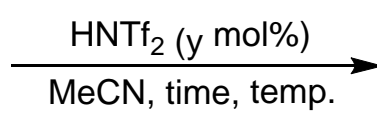
MeCN, time, temp.

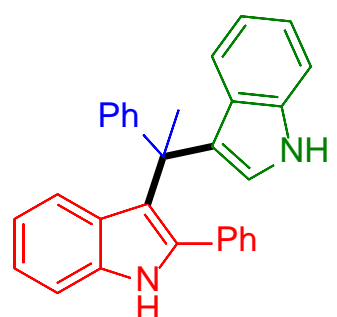

5d

Desired

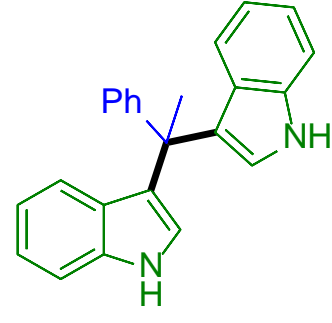

3b

Undesired

\begin{tabular}{llllllll} 
Entry & Temp $\left({ }^{\circ} \mathrm{C}\right)$ & Time (h) & $\mathbf{x}$ & $\mathbf{y}$ & $\begin{array}{l}\mathbf{4 f}(\%)^{\mathrm{a}} \\
\text { unreacted }\end{array}$ & $\mathbf{5 d}(\%)^{\mathrm{a}}$ & $\mathbf{3 b}(\mathbf{\%})^{\mathrm{a}}$ \\
\hline 1 & 30 & 24 & 1 & 5 & $39 \%$ & $0 \%$ & $43 \%$ \\
2 & 30 & 24 & 3 & 5 & $30 \%$ & $0 \%$ & $67 \%$ \\
3 & 30 & 24 & 5 & 5 & Quant. & $0 \%$ & $0 \%$ \\
4 & 30 & 24 & 3 & 10 & $18 \%$ & $0 \%$ & $\mathbf{7 2 \%}$ \\
5 & 50 & 24 & 3 & 5 & $48 \%$ & $0 \%$ & $49 \%$ \\
6 & 30 & 72 & 3 & 5 & $33 \%$ & $0 \%$ & $49 \%$ \\
7 & 30 & 24 & 3 & 20 & $6 \%$ & $0 \%$ & $47 \%$ \\
$8^{b}$ & 30 & 24 & 3 & 10 & $21 \%$ & $0 \%$ & $76 \%$ \\
9 & 30 & 48 & 3 & 10 & $21 \%$ & $0 \%$ & $\mathbf{7 2 \%}$ \\
$\mathbf{1 0}$ & $\mathbf{3 0}$ & $\mathbf{2 4}$ & $\mathbf{3}$ & $\mathbf{1 5}$ & $\mathbf{1 0 \%}$ & $\mathbf{0 \%}$ & $\mathbf{9 0 \%}(\mathbf{9 1 \% )}$ \\
\hline
\end{tabular}

All reactions carried out at $0.067 \mathrm{M}$ unless otherwise stated. ${ }^{\text {a }}$ ield determined by ${ }^{1} \mathrm{H}$ NMR analysis using dimethylsulfone as an internal standard. ${ }^{b} 0.2 \mathrm{M}$ concentration. Parentheses indicates isolated yields.

The same observation was noted even when a smaller substituent was present at the 2-position of vinyl indole 4 ( $\mathrm{Me}$ in $\mathbf{4 a}$ rather than $\mathrm{Ph}$ in $\mathbf{4 f}$ ). While the reaction proceeded to completion under milder conditions with $\mathbf{4 a}$ (acid $\mathbf{6}$ at $0{ }^{\circ} \mathrm{C}$ ) vs. $\mathbf{4 f}$, the symmetrical BIM $\mathbf{3 d}$ is still formed instead of the desired unsymmetrical BIM 5 (Scheme S-1).

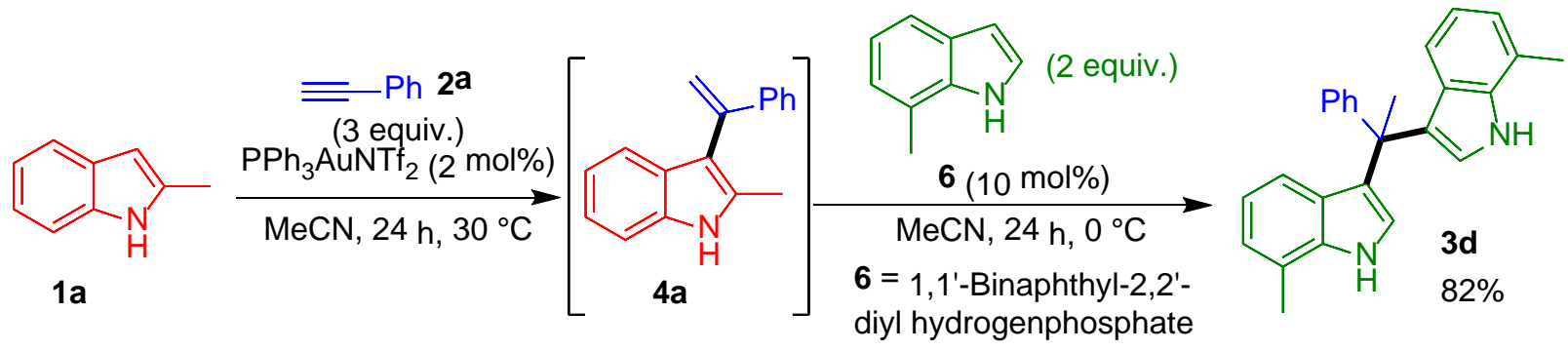

Scheme S-1 Substituent displacement of 2-substituted vinyl indoles 
Based on literature reports, ${ }^{1}$ the proposed mechanism for the formation of symmetrical BIM 3 from vinyl indole 4 via unsymmetrical BIM 5 is shown in Scheme S-2. Displacement of the sterically bulky indole (in red) from $\mathbf{5 d}$ presumably occurs because it forms the less sterically strained, and thus more thermodynamically favoured $\mathbf{3 b}$. Re-investigating the sequence using non-bulky vinylindole $\mathbf{4 0}$ instead of bulky $\mathbf{4 f}$ therefore successfully allows for formation of $\mathbf{5}$ over 3 (Scheme 5B).

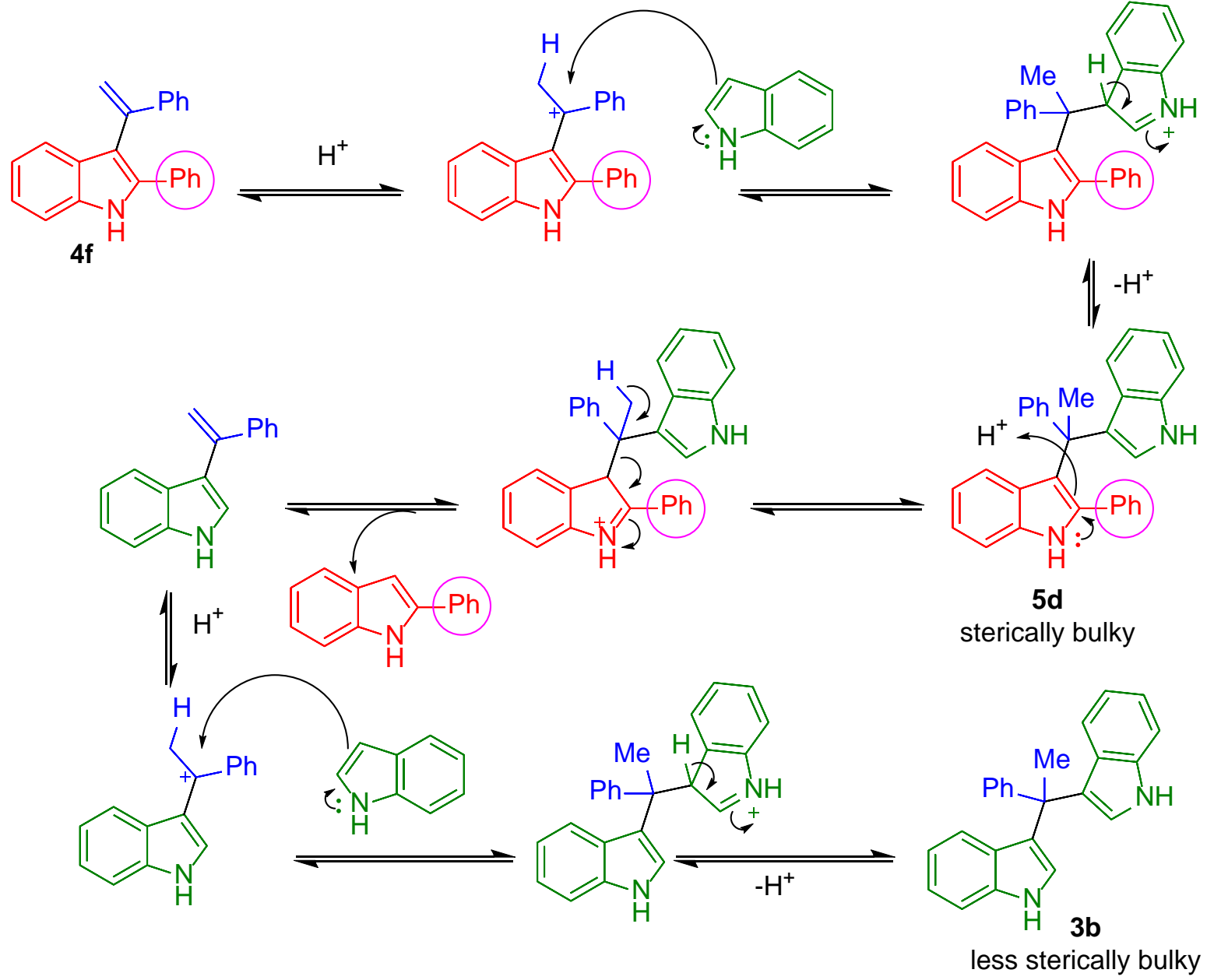

Scheme S-2 Proposed mechanism for formation of $\mathbf{3}$ 


\section{Starting Material Synthesis}

All starting materials were commercially available and purchased from Sigma Aldrich, Fluorochem or $\mathrm{TCl}$, apart from $\mathbf{1 c}$ and $\mathbf{1}$, which were synthesised using the procedures shown below. 2-Methylindole 1a was recrystallized prior to use (because an old bottle of 1a was used), but all other starting materials $\mathbf{1}$ and $\mathbf{2}$ were used as purchased without purification.

tert-Butyl 2-methyl-1H-indole-1-carboxylate (1c) ${ }^{2}$

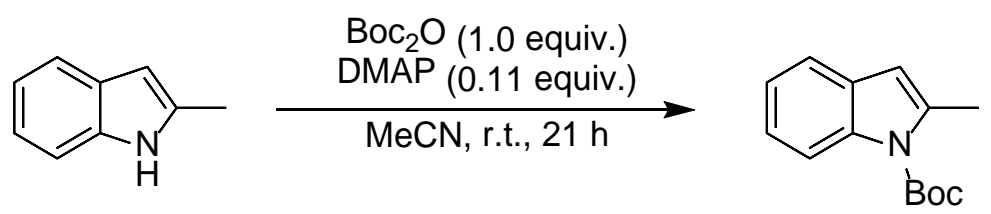

Following a procedure by Hecht, ${ }^{2}$ 2-methylindole $(741 \mathrm{mg}, 5.65 \mathrm{mmol})$, di-tert-butyl dicarbonate $(1.23 \mathrm{~g}, 5.65 \mathrm{mmol})$ and 4-dimethylaminopyridine $(67.0 \mathrm{mg}, 0.55 \mathrm{mmol})$ were dissolved in anhydrous $\mathrm{MeCN}(7.5 \mathrm{~mL})$. The resulting solution was stirred at room temperature for $21 \mathrm{~h}$. Upon completion of the reaction the solvent was removed under reduced pressure and the crude material redissolved in DCM $(20 \mathrm{~mL})$. The solution was then washed with saturated sodium bicarbonate solution $(20 \mathrm{~mL})$ followed by $\mathrm{H}_{2} \mathrm{O}(20 \mathrm{~mL})$. The organic layer was then dried over $\mathrm{MgSO}_{4}$ and filtered before the solvent was removed under reduced pressure. Purification of the crude residue by silica gel flash column chromatography (50:1 petrol 40$\left.60^{\circ} \mathrm{C} / \mathrm{EtOAc}\right)$ yielded the product $1 \mathrm{c}(1.124 \mathrm{~g}, 4.86 \mathrm{mmol}, 86 \%)$ as a white solid.

$\mathbf{R}_{\mathbf{f}} 0.30$ (50:1 petrol 40-60 ㄷ/EtOAC); $\mathbf{v}_{\max } / \mathrm{cm}^{-1} 3025$ (C-H Ar), 3010 (C-H Ar), 2983 (C-H), 2919 (C-H), 1720 (C=O), 1595 (C=C Ar), 1569 ( $C=C$ Ar), 1452 (C=C Ar), 1440 ( $C=C$ Ar) ; ${ }^{1} \mathrm{H}$ NMR (CDCl $400 \mathrm{MHz}) \delta_{\mathrm{H}} 8.12-8.08(\mathrm{~m}, 1 \mathrm{H}, \mathrm{Ar}-\mathrm{H}), 7.45-7.41(\mathrm{~m}, 1 \mathrm{H}, \mathrm{Ar}-\mathrm{H}), 7.25-7.15(\mathrm{~m}, 2 \mathrm{H}, \mathrm{Ar}-\mathrm{H})$,

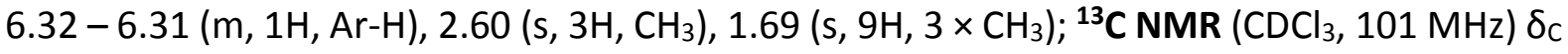
150.7 (C), 137.8 (C), 136.5 (C), $129.5(\mathrm{C}), 123.1(\mathrm{CH}), 122.6(\mathrm{CH}), 119.5(\mathrm{CH}), 115.5(\mathrm{CH}), 107.9$ $(\mathrm{CH}), 83.6(\mathrm{C}), 28.3\left(\mathrm{CH}_{3}\right), 17.1\left(\mathrm{CH}_{3}\right) ;$ m.p. $=52-54{ }^{\circ} \mathrm{C}\left(\mathrm{lit} .{ }^{3} 50-52{ }^{\circ} \mathrm{C}\right)$.

\section{2-(Trimethylsilyl)-1H-indole (1I)}

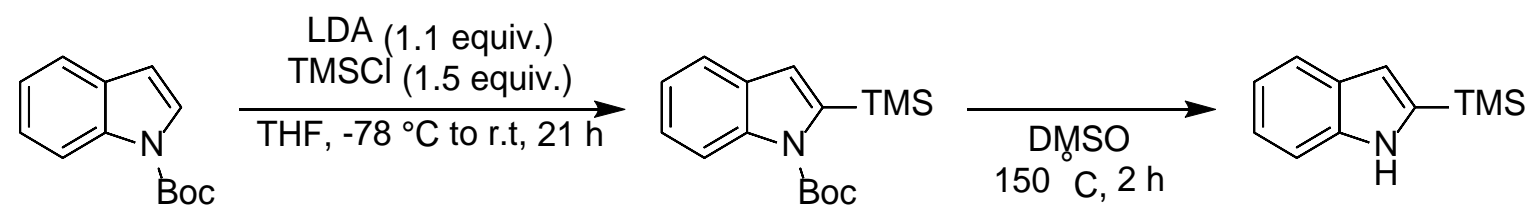

Adapting a procedure by Nielsen, ${ }^{4}$ a solution of diisopropylamine $(516 \mathrm{mg}, 720 \mu \mathrm{L}, 5.1 \mathrm{mmol}$ ) in anhydrous THF (20 mL) was cooled to $0{ }^{\circ} \mathrm{C}$ under an argon atmosphere, to this, $n$-butyl lithium solution (2.5M in hexane) $(2.04 \mathrm{~mL}, 5.1 \mathrm{mmol})$ was added dropwise and the resulting solution stirred at $0{ }^{\circ} \mathrm{C}$ for $1 \mathrm{~h}$. The previously prepared LDA solution was then added dropwise to a solution of tert-butyl $1 \mathrm{H}$-indole-1-carboxylate $(1.00 \mathrm{~g}, 935 \mu \mathrm{L}, 4.6 \mathrm{mmol})$ in anhydrous THF (54 $\mathrm{mL}$ ) at $-78^{\circ} \mathrm{C}$ under an argon atmosphere. The resulting solution was then stirred at $-78{ }^{\circ} \mathrm{C}$ for 
10 minutes. Chlorotrimethylsilane $(750 \mathrm{mg}, 878 \mu \mathrm{L}, 6.9 \mathrm{mmol})$ was then added dropwise; the solution was left stirring and allowed to slowly warm up from $-78^{\circ} \mathrm{C}$ to r.t. over $18 \mathrm{~h}$.

After this time the volatiles were removed under reduced pressure and the resulting residue dissolved in DMSO $(80 \mathrm{~mL})$. The resulting solution was then heated at $150{ }^{\circ} \mathrm{C}$ for $2 \mathrm{~h}$. The reaction mixture was then allowed to cool to r.t. before being poured over ice. The resulting mixture was then poured into a 1:1 mixture of $\mathrm{H}_{2} \mathrm{O} / \mathrm{Et}_{2} \mathrm{O}(60 \mathrm{~mL})$. The aqueous and organic layers were separated before the aqueous layer was extracted sequentially with $\mathrm{Et}_{2} \mathrm{O}(2 \times 80$ $\mathrm{mL})$ and EtOAc $(2 \times 80 \mathrm{~mL})$. The combined organic layers were then washed with $\mathrm{H}_{2} \mathrm{O}(4 \times 60$ $\mathrm{mL}$ ) and then brine $(4 \times 60 \mathrm{~mL})$ before being dried over $\mathrm{Na}_{2} \mathrm{SO}_{4}$, filtered and concentrated under reduced pressure to yield the crude product. Purification by silica gel flash column chromatography $\left(99: 1 \rightarrow 97: 3\right.$ petrol $\left.40-60^{\circ} \mathrm{C} / E t O A c\right)$ yielded the product 1 l ( $482.3 \mathrm{mg}, 2.53 \mathrm{mmol}$, $55 \%)$ as an orange oil which crystallised upon standing.

$\mathbf{R}_{\mathbf{f}} 0.23$ (97:3 petrol 40-60 ${ }^{\circ} \mathrm{C} /$ EtOAc); $\mathbf{v}_{\max } / \mathrm{cm}^{-1} 3404$ (N-H), 3025 (C-H Ar), 2955 (C-H), 2923 (C-H), 1497 (C=C Ar), 1443 (C=C Ar); ${ }^{1} \mathrm{H}$ NMR $\left(\mathrm{CDCl}_{3}, 400 \mathrm{MHz}\right) \delta_{\mathrm{H}} 8.12$ (br s, 1H, N-H), 7.66 (ddd, $J=8.0,1.9,0.9 \mathrm{~Hz}, 1 \mathrm{H}, \mathrm{Ar}-\mathrm{H}$ ), 7.42 (ddd, $J=8.1,1.9,0.9 \mathrm{~Hz}, 1 \mathrm{H}, \mathrm{Ar}-\mathrm{H}$ ), 7.21 (ddd, $J=8.1$, 7.0, $1.1 \mathrm{~Hz}, 1 \mathrm{H}, \mathrm{Ar}-\mathrm{H}$ ), 7.12 (ddd, J=8.0, 7.0, $1.1 \mathrm{~Hz}, 1 \mathrm{H}, \mathrm{Ar}-\mathrm{H}$ ), 6.76 (dd, J= 1.9, $1.1 \mathrm{~Hz}, 1 \mathrm{H}, \mathrm{Ar}-$ $\mathrm{H}), 0.38\left(\mathrm{~s}, 9 \mathrm{H}, 3 \times \mathrm{CH}_{3}\right) ;{ }^{13} \mathrm{C} \mathrm{NMR}\left(\mathrm{CDCl}_{3}, 75 \mathrm{MHz}\right) \delta_{\mathrm{c}} 138.6(\mathrm{C}), 138.2(\mathrm{C}), 128.6(\mathrm{C}), 122.2(\mathrm{CH})$, $120.5(\mathrm{CH}), 119.6(\mathrm{CH}), 111.3(\mathrm{CH}), 110.8(\mathrm{CH}),-1.1\left(\mathrm{CH}_{3}\right)$; m.p. $=46-48^{\circ} \mathrm{C}$. 


\section{General Procedures for the Gold(I)-Catalysed Hydroarylation of Alkynes}

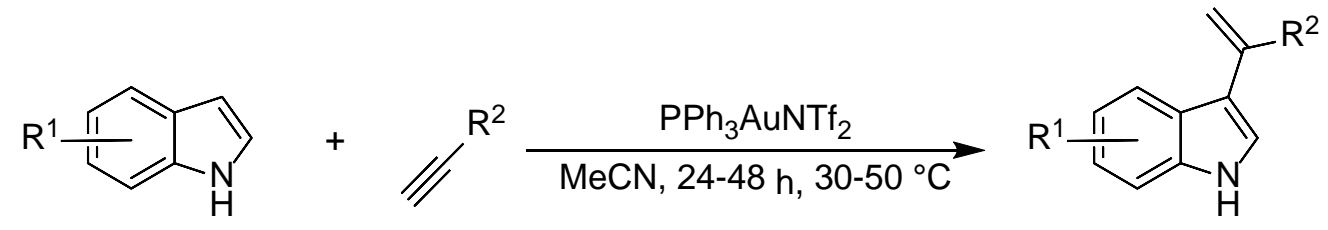

\section{General Procedure A:}

An oven-dried vial was charged with the indole $1(0.100 \mathrm{mmol}, 1$ equiv.), the alkyne $2(0.3$ mmol, 3 equiv.) and $\mathrm{PPh}_{3} \mathrm{AuNTf}_{2}$ (2:1 toluene adduct) $(1.6 \mathrm{mg}, 0.001 \mathrm{mmol}, 2 \mathrm{~mol} \%$ Au loading). The reagents were then dissolved in anhydrous $\mathrm{MeCN}(0.3 \mathrm{~mL})$. The resulting solution was then stirred at $30^{\circ} \mathrm{C}$ for $24 \mathrm{~h}$. Upon completion of the reaction, volatiles were removed under reduced pressure before the resulting crude residue was either purified using silica gel flash column chromatography to yield the products 4 , or taken forward to the next step without further purification.

\section{General Procedure B:}

Same as for general procedure B but with $\mathrm{PPh}_{3} \mathrm{AuNTf}_{2}$ (2:1 toluene adduct) (3.9 mg, 0.0025 mmol, 5 mol\% Au loading) and with a reaction temperature of $50{ }^{\circ} \mathrm{C}$.

\section{General Procedure C:}

Same as for general procedure B but with $\mathrm{PPh}_{3} \mathrm{AuNTf}_{2}$ (2:1 toluene adduct) $(7.9 \mathrm{mg}, 0.005$ mmol, 10 mol\% Au loading).

\section{General Procedure D:}

Same as for general procedure $C$ but with a reaction time of $48 \mathrm{~h}$.

\section{General Procedure E:}

Same as for general procedure $\mathrm{C}$ but with $\mathrm{PPh}_{3} \mathrm{AuNTf}_{2}$ (2:1 toluene adduct) (11.9 mg, 0.0075 mmol, 15 mol\% Au loading). 


\section{Product Characterisation}

\section{2-Methyl-3-(1-phenylvinyl)-1H-indole (4a) ${ }^{5}$}<smiles>C=C(c1ccccc1)c1c(C)[nH]c2ccccc12</smiles>

Following general procedure A, 2-methylindole $(13.1 \mathrm{mg}, 0.100 \mathrm{mmol})$ was reacted with phenylacetylene $(30.6 \mathrm{mg}, 33 \mu \mathrm{L}, 0.3 \mathrm{mmol})$. The crude mixture was purified by silica gel flash column chromatography (10:1 petrol $40-60^{\circ} \mathrm{C} /$ EtOAc) to yield product $4 a$ (19.5 $\mathrm{mg}, 0.084$ $\mathrm{mmol}, 84 \%)$ as a yellow oil.

$\mathbf{R}_{\mathbf{f}} 0.25$ (10:1 petrol 40-60 ${ }^{\circ} \mathrm{C} /$ EtOAC); $\boldsymbol{v}_{\max } / \mathrm{cm}^{-1} 3400(\mathrm{~N}-\mathrm{H}), 3057$ (Csp $\left.{ }^{2}-\mathrm{H}\right), 3007$ (Csp $\left.{ }^{2}-\mathrm{H}\right), 2917$ (Csp $\left.{ }^{3}-\mathrm{H}\right), 1619$ (C=C), 1599 (C=C Ar), 1492 (C=C Ar), 1459 (C=C Ar); ${ }^{1} \mathrm{H}_{\mathrm{NMR}}\left(\mathrm{CDCl}_{3}, 300 \mathrm{MHz}\right)$ $\delta_{\mathrm{H}} 7.90(\mathrm{~s}, 1 \mathrm{H}, \mathrm{Ar}-\mathrm{H}), 7.46-7.39(\mathrm{~m}, 2 \mathrm{H}, \mathrm{Ar}-\mathrm{H}), 7.34-7.28(\mathrm{~m}, 4 \mathrm{H}, \mathrm{Ar}-\mathrm{H}), 7.26-7.22(\mathrm{~m}, 1 \mathrm{H}$, Ar-H), 7.14 (ddd, $J=8.1,7.1,1.2 \mathrm{~Hz}, 1 \mathrm{H}, \mathrm{Ar}-\mathrm{H}$ ), 7.01 (ddd, $J=8.1,7.1,1.2 \mathrm{~Hz}, 1 \mathrm{H}, \mathrm{Ar}-\mathrm{H}$ ), 5.76 $(\mathrm{d}, J=1.9 \mathrm{~Hz}, 1 \mathrm{H}, \mathrm{C}=\mathrm{CHH}), 5.36(\mathrm{~d}, J=1.9 \mathrm{~Hz}, 1 \mathrm{H}, \mathrm{C}=\mathrm{CHH}), 2.29\left(\mathrm{~s}, 3 \mathrm{H}, \mathrm{CH}_{3}\right) ;{ }^{13} \mathrm{C} \mathrm{NMR}\left(\mathrm{CDCl}_{3}\right.$, $75 \mathrm{MHz}$ ) $\delta_{\mathrm{C}} 142.5$ (C), 141.9 (C), 135.1 (C), 133.1 (C), 128.4 (C), $128.2(\mathrm{CH}), 127.5(\mathrm{CH}), 127.3$ $(\mathrm{CH}), 121.2(\mathrm{CH}), 119.7(\mathrm{CH}), 119.6(\mathrm{CH}), 114.9\left(\mathrm{CH}_{2}\right), 114.0(\mathrm{C}), 110.1(\mathrm{CH}), 12.8\left(\mathrm{CH}_{3}\right)$.

\section{1,2-Dimethyl-3-(1-phenylvinyl)-1H-indole (4b) ${ }^{6}$}<smiles>C=C(c1ccccc1)c1c(C)n(C)c2ccccc12</smiles>

Following general procedure A, 1,2-dimethylindole $(14.5 \mathrm{mg}, 0.100 \mathrm{mmol})$ was reacted with phenylacetylene $(30.6 \mathrm{mg}, 33 \mu \mathrm{L}, 0.3 \mathrm{mmol})$. The crude mixture was purified by silica gel flash column chromatography $\left(25: 1\right.$ petrol $40-60^{\circ} \mathrm{C} /$ EtOAC) to yield product $4 \mathrm{~b}$ ( $22.4 \mathrm{mg}, 0.091$ $\mathrm{mmol}, 91 \%)$ as a yellow solid.

$\mathbf{R}_{\mathbf{f}} 0.37$ (20:1 petrol 40-60 $\left.{ }^{\circ} \mathrm{C} / \mathrm{EtOAC}\right) ; \mathbf{v}_{\max } / \mathrm{cm}^{-1} 3059$ (Csp $\left.{ }^{2}-\mathrm{H}\right), 3026\left(\mathrm{Csp}^{2}-\mathrm{H}\right), 2931\left(\mathrm{Csp}^{3}-\mathrm{H}\right)$, $2921\left(\mathrm{Csp}^{3}-\mathrm{H}\right), 1611$ (C=C), 1472 ( $\left.\mathrm{C}=\mathrm{C} \mathrm{Ar}\right), 1452$ (C=C Ar), 1375 (C=C Ar); ${ }^{1} \mathbf{H ~ N M R}\left(\mathrm{CDCl}_{3}, 400\right.$ $\mathrm{MHz}) \delta_{H} 7.41-7.36(\mathrm{~m}, 2 \mathrm{H}, \mathrm{Ar}-\mathrm{H}), 7.30-7.26(\mathrm{~m}, 2 \mathrm{H}, \mathrm{Ar}-\mathrm{H}), 7.26-7.20(\mathrm{~m}, 3 \mathrm{H}, \mathrm{Ar}-\mathrm{H}), 7.14$ (dd, J=8.6, 1.1 Hz, 1H, Ar-H), 6.97 (dd, J=8.1, 1.1 Hz, 1H, Ar-H), $5.74(\mathrm{~d}, J=1.9 \mathrm{~Hz}, 1 \mathrm{H}, \mathrm{C}=\mathrm{CHH})$, $5.29(\mathrm{~d}, J=1.9 \mathrm{~Hz}, 1 \mathrm{H}, \mathrm{C}=\mathrm{CHH}), 3.69\left(\mathrm{~s}, 3 \mathrm{H}, \mathrm{NCH}_{3}\right), 2.26\left(\mathrm{~s}, 3 \mathrm{H}, \mathrm{CH}_{3}\right) ;{ }^{13} \mathrm{C} \mathrm{NMR}\left(\mathrm{CDCl}_{3}, 101 \mathrm{MHz}\right)$ $\delta_{\mathrm{C}} 142.8(\mathrm{C}), 142.1(\mathrm{C}), 136.6(\mathrm{C}), 134.9(\mathrm{C}), 128.1(\mathrm{CH}), 127.6(\mathrm{C}), 127.4(\mathrm{CH}), 127.3(\mathrm{CH}), 120.8$ $(\mathrm{CH}), 119.6(\mathrm{CH}), 119.3(\mathrm{CH}), 114.9\left(\mathrm{CH}_{2}\right), 113.5(\mathrm{C}), 108.5(\mathrm{CH}), 29.6\left(\mathrm{CH}_{3}\right), 11.4\left(\mathrm{CH}_{3}\right)$; m.p. = 74-75 ${ }^{\circ} \mathrm{C}$ (lit. $\left.{ }^{6} 76-77^{\circ} \mathrm{C}\right)$. 
<smiles>C=C(c1ccccc1)c1c(C)[nH]c2ccc(Cl)cc12</smiles>

Following general procedure A, 5-chloro-2-methylindole $(16.6 \mathrm{mg}, 0.100 \mathrm{mmol})$ was reacted with phenylacetylene $(30.6 \mathrm{mg}, 33 \mu \mathrm{L}, 0.3 \mathrm{mmol})$. The crude mixture was purified by silica gel flash column chromatography $\left(9: 1\right.$ petrol $40-60^{\circ} \mathrm{C} /$ EtOAc) to yield product $4 \mathbf{d}(24.2 \mathrm{mg}, 0.090$ $\mathrm{mmol}, 90 \%)$ as a yellow oil.

$\mathbf{R}_{\mathbf{f}} 0.20$ (9:1 petrol 40-60 $\left.{ }^{\circ} \mathrm{C} / \mathrm{EtOAC}\right) ; \mathbf{v}_{\max } / \mathrm{cm}^{-1} 3416(\mathrm{~N}-\mathrm{H}), 3059$ (Csp $\left.{ }^{2}-\mathrm{H}\right), 3017\left(\mathrm{Csp}^{2}-\mathrm{H}\right), 2916$ (Csp $\left.{ }^{3}-\mathrm{H}\right), 1606$ (C=C), 1572 (C=C Ar), 1470 (C=C Ar), 1446 (C=C Ar); ${ }^{1} \mathrm{H}_{\mathrm{NMR}}\left(\mathrm{CDCl}_{3}, 400 \mathrm{MHz}\right)$ $\delta_{H} 7.94(\mathrm{~s}, 1 \mathrm{H}, \mathrm{Ar}-\mathrm{H}), 7.40-7.36(\mathrm{~m}, 2 \mathrm{H}, \mathrm{Ar}-\mathrm{H}), 7.33-7.29(\mathrm{~m}, 3 \mathrm{H}, \mathrm{Ar}-\mathrm{H}), 7.23-7.19(\mathrm{~m}, 2 \mathrm{H}$, $\operatorname{Ar}-\mathrm{H}$ ), 7.07 (dd, J=8.5, 2.0 Hz, 1H, Ar-H), $5.76(\mathrm{~d}, J=1.7 \mathrm{~Hz}, 1 \mathrm{H}, \mathrm{C}=\mathrm{CHH}), 5.33(\mathrm{~d}, J=1.7 \mathrm{~Hz}$, $1 \mathrm{H}, \mathrm{C}=\mathrm{CHH}), 2.25\left(\mathrm{~s}, 3 \mathrm{H}, \mathrm{CH}_{3}\right) ;{ }^{13} \mathrm{C}$ NMR $\left(\mathrm{CDCl}_{3}, 101 \mathrm{MHz}\right) \delta_{\mathrm{c}} 141.9$ (C), $141.4(\mathrm{C}), 134.5$ (C), $133.5(\mathrm{C}), 129.6(\mathrm{C}), 128.3(\mathrm{CH}), 127.7(\mathrm{CH}), 127.2(\mathrm{CH}), 125.4(\mathrm{C}), 121.5(\mathrm{CH}), 119.0(\mathrm{CH}), 115.4$ $\left(\mathrm{CH}_{2}\right), 114.0(\mathrm{C}), 111.1(\mathrm{CH}), 12.8\left(\mathrm{CH}_{3}\right) ; \mathrm{HRMS}(\mathrm{FTMS}+\mathrm{p} \mathrm{NSI}) \mathrm{m} / \mathrm{z}:[\mathrm{M}+\mathrm{H}]^{+}$Calcd for $\mathrm{C}_{17} \mathrm{H}_{15} \mathrm{NCl}$ 268.0888; Found 268.0887.

\section{5-Methoxy-2-methyl-3-(1-phenylvinyl)-1H-indole (4e)}<smiles>C=C(c1ccccc1)c1c(C)[nH]c2ccc(OC)cc12</smiles>

Following general procedure $A, 5$-methoxy-2-methyl- $1 H$-indole $(16.1 \mathrm{mg}, 0.100 \mathrm{mmol}$ ) was reacted with phenylacetylene $(30.6 \mathrm{mg}, 33 \mu \mathrm{L}, 0.3 \mathrm{mmol})$. The crude mixture was purified by silica gel flash column chromatography (10:1 hexane/EtOAc) to yield product 4 e $(20.2 \mathrm{mg}$, $0.077 \mathrm{mmol}, 77 \%$ ) as a green solid.

$\mathbf{R}_{\mathbf{f}} 0.23$ (10:1 hexane/EtOAc); $\mathbf{v}_{\max } / \mathrm{cm}^{-1} 3398(\mathrm{~N}-\mathrm{H}), 3079\left(\mathrm{Csp}^{2}-\mathrm{H}\right), 3054$ (Csp $\left.{ }^{2}-\mathrm{H}\right), 2994$ (Csp ${ }^{3}-$ H), 2936 ( $\left.\mathrm{Csp}^{3}-\mathrm{H}\right), 2829$ ( $\left.\mathrm{Csp}^{3}-\mathrm{H}\right), 1677$ (C=C), 1623 (C=C Ar), 1598 (C=C Ar), 1582 (C=C Ar), 1483 (C=C Ar), 1464 (C=C Ar), 1449 (C=C Ar), 1276 (C-O-Ar), 1111 (C-O-C); ${ }^{1} \mathbf{H}$ NMR (CDCl, 400 $\mathrm{MHz}) \delta_{\mathrm{H}} 7.82(\mathrm{br} \mathrm{s}, 1 \mathrm{H}, \mathrm{N}-\mathrm{H}), 7.44-7.40(\mathrm{~m}, 2 \mathrm{H}, \mathrm{Ar}-\mathrm{H}), 7.34-7.28(\mathrm{~m}, 3 \mathrm{H}, \mathrm{Ar}-\mathrm{H}), 7.19$ (dd, $J=$ 8.7, $0.6 \mathrm{~Hz}, 1 \mathrm{H}, \mathrm{Ar}-\mathrm{H}), 6.78$ (dd, $J=8.7,2.5 \mathrm{~Hz}, 1 \mathrm{H}, \mathrm{Ar}-\mathrm{H}), 6.70$ (dd, $J=2.5,0.6 \mathrm{~Hz}, 1 \mathrm{H}, \mathrm{Ar}-\mathrm{H}$ ), $5.74(\mathrm{~d}, J=1.9 \mathrm{~Hz}, 1 \mathrm{H}, \mathrm{C}=\mathrm{CHH}), 5.34(\mathrm{~d}, J=1.9 \mathrm{~Hz}, 1 \mathrm{H}, \mathrm{C}=\mathrm{CHH}), 3.70\left(\mathrm{~s}, 3 \mathrm{H}, \mathrm{OCH}_{3}\right), 2.26(\mathrm{~s}, 3 \mathrm{H}$, $\left.\mathrm{CH}_{3}\right) ;{ }^{13} \mathrm{C}$ NMR $\left(\mathrm{CDCl}_{3}, 101 \mathrm{MHz}\right) \delta_{\mathrm{c}} 154.2$ (C), 142.7 (C), 141.8 (C), 133.9 (C), 130.3 (C), 129.0 (C), $128.2(\mathrm{CH}), 127.5(\mathrm{CH}), 127.3(\mathrm{CH}), 114.6\left(\mathrm{CH}_{2}\right), 114.1(\mathrm{C}), 111.0(\mathrm{CH}), 110.7(\mathrm{CH}), 102.2$ (CH), $55.8\left(\mathrm{CH}_{3}\right), 12.9\left(\mathrm{CH}_{3}\right) ;$ m.p. $=135-137^{\circ} \mathrm{C}$; HRMS (FTMS + c ESI) m/z: $[\mathrm{M}+\mathrm{H}]^{+}$Calcd for $\mathrm{C}_{18} \mathrm{H}_{18} \mathrm{ON}$ 264.1383; Found 264.1382. 


\section{2-Phenyl-3-(1-phenylvinyl)-1H-indole (4f) ${ }^{7}$}<smiles>C=C(c1ccccc1)c1c(-c2ccccc2)[nH]c2ccccc12</smiles>

Following general procedure A, 2-phenylindole $(19.3 \mathrm{mg}, 0.100 \mathrm{mmol})$ was reacted with phenylacetylene $(30.6 \mathrm{mg}, 33 \mu \mathrm{L}, 0.3 \mathrm{mmol})$. The crude mixture was purified by silica gel flash column chromatography (10:1 hexane/EtOAc) to yield product $\mathbf{4 f}(28.4 \mathrm{mg}, 0.096 \mathrm{mmol}, 96 \%)$ as a yellow solid.

\section{$1 \mathrm{Mmol}$ Scale}

An oven-dried flask was charged with 2-methylindole (193 mg, $1.00 \mathrm{mmol}$ ), phenylacetylene (306 mg, $330 \mu \mathrm{L}, 3 \mathrm{mmol}$ ) and PPh ${ }_{3} \mathrm{AuNTf}_{2}$ (2:1 toluene adduct) $(16.0 \mathrm{mg}, 0.01 \mathrm{mmol}, 2 \mathrm{~mol} \%$ Au loading). The reagents were dissolved in anhydrous $\operatorname{MeCN}(3 \mathrm{~mL})$ and the resulting reaction mixture was then stirred at $30{ }^{\circ} \mathrm{C}$ for $24 \mathrm{~h}$. Upon completion of the reaction, volatiles were removed under reduced pressure before purification of the crude residue of by silica gel flash column chromatography $\left(10: 1\right.$ petrol $\left.40-60{ }^{\circ} \mathrm{C} / \mathrm{EtOAC}\right)$ gave the product $\mathbf{4 f}$ ( $295.4 \mathrm{mg}$, $1.00 \mathrm{mmol}$, quant.) as a yellow solid.

$\mathbf{R}_{\mathbf{f}} 0.24$ (10:1 hexane/EtOAC); $\mathbf{v}_{\max } / \mathrm{cm}^{-1} 3412(\mathrm{~N}-\mathrm{H}), 3057$ (Csp $\left.{ }^{2}-\mathrm{H}\right), 3024$ (Csp $\left.{ }^{2}-\mathrm{H}\right), 1603$ (C=C), 1573 (C=C Ar), 1489 (C=C Ar), 1448 (C=C Ar); ${ }^{1} \mathrm{H}$ NMR (CDCl, $400 \mathrm{MHz}$ ) $\delta_{\mathrm{H}} 8.23$ (br s, 1H, N$\mathrm{H}), 7.60-7.56(\mathrm{~m}, 2 \mathrm{H}, \mathrm{Ar}-\mathrm{H}), 7.49-7.45(\mathrm{~m}, 2 \mathrm{H}, \mathrm{Ar}-\mathrm{H}), 7.43$ (ddd, J = 8.0, $0.9 \mathrm{~Hz}, 1 \mathrm{H}, \mathrm{Ar}-\mathrm{H})$, $7.36(\mathrm{dm}, J=8.0 \mathrm{~Hz}, 1 \mathrm{H}, \mathrm{Ar}-\mathrm{H}), 7.34-7.29(\mathrm{~m}, 2 \mathrm{H}, \mathrm{Ar}-\mathrm{H}), 7.27-7.20(\mathrm{~m}, 5 \mathrm{H}, \mathrm{Ar}-\mathrm{H}), 7.08$ (ddd, $J=8.0,7.0,0.9 \mathrm{~Hz}, 1 \mathrm{H}, \mathrm{Ar}-\mathrm{H}), 5.85(\mathrm{~d}, J=1.6 \mathrm{~Hz}, 1 \mathrm{H}, \mathrm{C}=\mathrm{C}-\mathrm{HH}), 5.38(\mathrm{~d}, J=1.7 \mathrm{~Hz}, 1 \mathrm{H}, \mathrm{C}=\mathrm{C}-\mathrm{HH})$; ${ }^{13} \mathrm{C} \mathrm{NMR}\left(\mathrm{CDCl}_{3}, 101 \mathrm{MHz}\right) \delta_{\mathrm{C}} 142.1$ (C), 141.0 (C), 135.8 (C), 135.2 (C), 132.6 (C), 129.5 (C), $128.5(\mathrm{CH}), 128.1(\mathrm{CH}), 127.6(\mathrm{CH}), 127.6(\mathrm{CH}), 127.5(\mathrm{CH}), 127.0(\mathrm{CH}), 122.5(\mathrm{CH}), 120.3(\mathrm{CH})$, $120.2(\mathrm{CH}), 116.8\left(\mathrm{CH}_{2}\right), 114.8(\mathrm{C}), 110.7(\mathrm{CH}) ;$ m.p. $=45-47^{\circ} \mathrm{C}$ (lit. ${ }^{7} 43-45^{\circ} \mathrm{C}$ ).

\section{2-(tert-Butyl)-3-(1-phenylvinyl)-1H-indole (4g)}<smiles>C=C(c1ccccc1)c1c(C(C)(C)C)[nH]c2ccccc12</smiles>

Following general procedure B, 2-(tert-butyl)- $1 H$-indole $(16.1 \mathrm{mg}, 0.100 \mathrm{mmol})$ was reacted with phenylacetylene $(30.6 \mathrm{mg}, 33 \mu \mathrm{L}, 0.3 \mathrm{mmol})$. The crude mixture was purified by silica gel flash column chromatography (10:1 hexane/EtOAc) to yield product $4 \mathrm{~g}(27.5 \mathrm{mg}, 0.100 \mathrm{mmol}$, quant.) as a brown oil.

$\mathbf{R}_{\mathbf{f}} 0.33$ (10:1 hexane/EtOAc); $\mathbf{v}_{\max } / \mathrm{cm}^{-1} 3479(\mathrm{~N}-\mathrm{H}), 3059$ (Csp $\left.{ }^{2}-\mathrm{H}\right), 3026$ (Csp $\left.{ }^{2}-\mathrm{H}\right), 2964$ (Csp ${ }^{3}-$ H), 2922 (Csp $\left.{ }^{3}-\mathrm{H}\right), 2867$ (Csp $\left.{ }^{3}-\mathrm{H}\right), 1614$ (C=C), 1493 (C=C Ar), 1452 (C=C Ar); ${ }^{1} \mathrm{H} \mathrm{NMR}$ (CDCl $_{3}$, $300 \mathrm{MHz}$ ) $\delta_{\mathrm{H}} 8.03$ (br s, $\left.1 \mathrm{H}, \mathrm{N}-\mathrm{H}\right), 7.45-7.39(\mathrm{~m}, 2 \mathrm{H}, \mathrm{Ar}-\mathrm{H}), 7.38-7.32(\mathrm{~m}, 2 \mathrm{H}, \mathrm{Ar}-\mathrm{H}), 7.31-$ 
$7.23(\mathrm{~m}, 3 \mathrm{H}, \mathrm{Ar}-\mathrm{H}), 7.17$ (ddd, J = 8.1, 7.0, $1.1 \mathrm{~Hz}, 1 \mathrm{H}, \mathrm{Ar}-\mathrm{H}), 7.04$ (ddd, J = 8.1, 7.1, $1.1 \mathrm{~Hz}, 1 \mathrm{H}$, $\operatorname{Ar}-\mathrm{H}), 6.05(\mathrm{~d}, J=1.8 \mathrm{~Hz}, 1 \mathrm{H}, \mathrm{C}=\mathrm{CHH}), 5.38(\mathrm{~d}, \mathrm{~J}=1.8 \mathrm{~Hz}, 1 \mathrm{H}, \mathrm{C}=\mathrm{CHH}), 1.36\left(\mathrm{~s}, 9 \mathrm{H}, 3 \times \mathrm{CH}_{3}\right) ;{ }^{13} \mathrm{C}$ NMR (CDCl, $75 \mathrm{MHz}) \delta_{\mathrm{C}} 143.1$ (C), 142.5 (C), 141.8 (C), 133.9 (C), 130.3 (C), 128.1 (CH), 127.3 $(\mathrm{CH}), 126.6(\mathrm{CH}), 121.4(\mathrm{CH}), 119.6(\mathrm{CH}), 119.1(\mathrm{CH}), 117.2\left(\mathrm{CH}_{2}\right), 112.5(\mathrm{C}), 110.0(\mathrm{CH}), 33.3$ (C), $30.5\left(\mathrm{CH}_{3}\right)$; HRMS (FTMS + C ESI) m/z: $[\mathrm{M}+\mathrm{H}]^{+}$Calcd for $\mathrm{C}_{20} \mathrm{H}_{22} \mathrm{~N}$ 276.1747; Found 276.1744 .

\section{Ethyl 2-(3-(1-phenylvinyl)-1H-indol-2-yl)acetate (4i)}<smiles>C=C(c1ccccc1)c1c(CC(=O)OCC)[nH]c2ccccc12</smiles>

Following general procedure $B$, ethyl $2-(1 H$-indol-2-yl)acetate $(20.3 \mathrm{mg}, 0.100 \mathrm{mmol})$ was reacted with phenylacetylene $(30.6 \mathrm{mg}, 33 \mu \mathrm{L}, 0.3 \mathrm{mmol})$. The crude mixture was purified by silica gel flash column chromatography (10:1 hexane/EtOAc) to yield product $4 \mathbf{i}(27.0 \mathrm{mg}$, $0.089 \mathrm{mmol}, 89 \%$ ) as a brown oil.

$\mathbf{R}_{\mathbf{f}} 0.25$ (10:1 hexane/EtOAc); $\mathbf{v}_{\max } / \mathrm{cm}^{-1} 3404(\mathrm{~N}-\mathrm{H}), 3059$ (Csp $\left.{ }^{2}-\mathrm{H}\right), 3014$ (Csp $\left.{ }^{2}-\mathrm{H}\right), 2983$ (Csp 3 H), 2928 ( $\mathrm{Csp}^{3}-\mathrm{H}$ ), 1723 (C=O), 1605 ( $\left.\mathrm{C}=\mathrm{C}\right), 1573$ (C=C Ar), 1492 (C=C Ar), 1458 (C=C Ar), 1179 (C-O-C); ${ }^{1} \mathrm{H} \mathrm{NMR}\left(\mathrm{CDCl}_{3}, 400 \mathrm{MHz}\right) \delta_{\mathrm{H}} 8.88(\mathrm{br} \mathrm{s}, 1 \mathrm{H}, \mathrm{N}-\mathrm{H}), 7.42-7.39(\mathrm{~m}, 2 \mathrm{H}, \mathrm{Ar}-\mathrm{H}), 7.37$ (ddd, $J=8.0,0.7,0.7 \mathrm{~Hz}, 1 \mathrm{H}, \mathrm{Ar}-\mathrm{H}), 7.33-7.28(\mathrm{~m}, 3 \mathrm{H}, \mathrm{Ar}-\mathrm{H}), 7.23(\mathrm{dm}, J=8.0 \mathrm{~Hz}, 1 \mathrm{H}, \mathrm{Ar}-\mathrm{H}), 7.17$ (ddd, $J=8.0,7.1,1.1 \mathrm{~Hz}, 1 \mathrm{H}, \mathrm{Ar}-\mathrm{H}$ ), 7.01 (ddd, $J=8.0,7.1,1.1 \mathrm{~Hz}, 1 \mathrm{H}, \mathrm{Ar}-\mathrm{H}$ ), 5.80 (d, J=1.8 Hz, $1 \mathrm{H}, \mathrm{C}=\mathrm{CHH}), 5.36(\mathrm{~d}, J=1.8 \mathrm{~Hz}, 1 \mathrm{H}, \mathrm{C}=\mathrm{CHH}), 4.18\left(\mathrm{q}, J=7.1 \mathrm{~Hz}, 2 \mathrm{H}, \mathrm{CH}_{2} \mathrm{CH}_{3}\right), 3.73\left(\mathrm{~s}, 2 \mathrm{H}, \operatorname{ArCH}_{2}\right)$, $1.27\left(\mathrm{t}, J=7.1 \mathrm{~Hz}, 3 \mathrm{H}, \mathrm{CH}_{3}\right) ;{ }^{13} \mathrm{C} \mathrm{NMR}\left(\mathrm{CDCl}_{3}, 101 \mathrm{MHz}\right) \delta_{\mathrm{c}} 170.8(\mathrm{C}), 141.9$ (C), 141.5 (C), 135.6 (C), $128.5(\mathrm{C}), 128.3(\mathrm{CH}), 127.8(\mathrm{C}), 127.7(\mathrm{CH}), 127.2(\mathrm{CH}), 122.0(\mathrm{CH}), 120.1(\mathrm{CH}), 119.8(\mathrm{CH})$, $115.7(\mathrm{C}), 115.5\left(\mathrm{CH}_{2}\right), 110.7(\mathrm{CH}), 61.3\left(\mathrm{CH}_{2}\right), 32.3\left(\mathrm{CH}_{2}\right), 14.1\left(\mathrm{CH}_{3}\right) ; \mathrm{HRMS}(\mathrm{FTMS}+\mathrm{c} \mathrm{ESI}) \mathrm{m} / \mathrm{z}$ : $[\mathrm{M}+\mathrm{H}]^{+}$Calcd for $\mathrm{C}_{20} \mathrm{H}_{20} \mathrm{O}_{2} \mathrm{~N}$ 306.1489; Found 306.1484.

\section{4-Bromo-3-(1-phenylvinyl)-1H-indole (4j)}<smiles>C=C(c1ccccc1)c1c[nH]c2cccc(Br)c12</smiles>

Following general procedure C, 4-bromoindole $(19.6 \mathrm{mg}, 0.100 \mathrm{mmol})$ was reacted with phenyl acetylene ( $30.6 \mathrm{mg}, 33 \mu \mathrm{L}, 0.3 \mathrm{mmol}$ ). The crude mixture was purified by silica gel flash column chromatography $(12: 1 \rightarrow 10: 1$ hexane/EtOAc) to yield product $4 \mathbf{j}$ (23.8 $\mathrm{mg}, 0.080$ $\mathrm{mmol}, 80 \%)$ as a yellow oil.

$\mathbf{R}_{\mathbf{f}} 0.17$ (12:1 hexane/EtOAc); $\mathbf{v}_{\max } / \mathrm{cm}^{-1} 3418(\mathrm{~N}-\mathrm{H}), 3079$ (Csp $\left.{ }^{2}-\mathrm{H}\right), 3055$ (Csp $\left.{ }^{2}-\mathrm{H}\right), 3025$ (Csp ${ }^{2}$ $\mathrm{H}), 1608$ ( $\mathrm{C}=\mathrm{C}), 1573$ ( $\mathrm{C}=\mathrm{C} \mathrm{Ar}), 1557$ (C=C Ar), 1537 (C=C Ar), 1492 (C=C Ar), 1480 (C=C Ar); ${ }^{1} \mathrm{H}$ 
NMR $\left(\mathrm{CDCl}_{3}, 400 \mathrm{MHz}\right) \delta_{\mathrm{H}} 8.13(\mathrm{br} \mathrm{s}, 1 \mathrm{H}, \mathrm{N}-\mathrm{H}), 7.30-7.22(\mathrm{~m}, 3 \mathrm{H}, \mathrm{Ar}-\mathrm{H}), 7.18-7.10(\mathrm{~m}, 5 \mathrm{H}$, Ar-H), 6.93 (dd, J = 7.9, 7.9 Hz, 1H, Ar-H), 5.75 (d, J=1.7 Hz, 1H, C=CHH), $5.27(\mathrm{~d}, J=1.7 \mathrm{~Hz}$, $1 \mathrm{H}, \mathrm{C}=\mathrm{CHH}) ;{ }^{13} \mathrm{C} \mathrm{NMR}\left(\mathrm{CDCl}_{3}, 75 \mathrm{MHz}\right) \delta_{\mathrm{C}} 142.3(\mathrm{C}), 142.3(\mathrm{C}), 137.1(\mathrm{C}), 128.0(\mathrm{CH}), 127.3(\mathrm{CH})$, $126.8(\mathrm{CH}), 125.6(\mathrm{C}), 125.3(\mathrm{CH}), 124.5(\mathrm{CH}), 123.1(\mathrm{CH}), 118.7(\mathrm{C}), 116.0\left(\mathrm{CH}_{2}\right), 114.7(\mathrm{C})$, $110.4(\mathrm{CH})$; HRMS (TOF MS ASAP+) m/z: $[\mathrm{M}+\mathrm{H}]^{+}$Calcd for $\mathrm{C}_{16} \mathrm{H}_{13} \mathrm{NBr} 298.0231$; Found 298.0231.

\section{3-Methyl-2-(1-phenylvinyl)-1H-indole (4k) ${ }^{8}$}<smiles>C=C(c1ccccc1)c1[nH]c2ccccc2c1C</smiles>

Following general procedure $A$ at $50{ }^{\circ} \mathrm{C}, 3$-methylindole $(13.1 \mathrm{mg}, 0.100 \mathrm{mmol}$ ) was reacted with phenylacetylene ( $30.6 \mathrm{mg}, 33 \mu \mathrm{L}, 0.3 \mathrm{mmol}$ ). After the reaction was complete, volatiles were removed under reduced pressure. ${ }^{1} \mathrm{H}$ NMR analysis of the crude mixture using 2,6-dimethoxy-14-benzoquinone as an internal standard showed formation of product $\mathbf{4 k}$ in a $75 \%$ yield. Attempts to purify $\mathbf{4 k}$ proved unsuccessful due to co-elution with unreacted 3-methylindole starting material. Several combinations of different catalyst loadings and reaction temperatures were screened, but conditions could not be found to push the reaction to completion.

\section{3-(1-Phenylvinyl)-2-(4,4,5,5-tetramethyl-1,3,2-dioxaborolan-2-yl)-1H-indole (4m)}<smiles>C=C(c1ccccc1)c1c(B2OC(C)(C)C(C)(C)O2)[nH]c2ccccc12</smiles>

Following general procedure B, 2-(4,4,5,5-tetramethyl-1,3,2-dioxaborolan-2-yl)- $1 H$-indole (24.3 $\mathrm{mg}, 0.100 \mathrm{mmol}$ ) was reacted with phenylacetylene (30.6 mg, $33 \mu \mathrm{L}, 0.3 \mathrm{mmol})$. After the reaction was complete, volatiles were removed under reduced pressure. ${ }^{1} \mathrm{H}$ NMR analysis of the crude mixture using dimethylsulfone as an internal standard showed formation of product $\mathbf{4 m}$ in an $85 \%$ yield. Attempts to purify $\mathbf{4 m}$ proved unsuccessful due to its instability on silica. 
<smiles>C=C(c1ccccc1)c1c(B2OC(C)(C)C(C)(C)O2)n(C)c2ccccc12</smiles>

Following general procedure C, 1-methyl-2-(4,4,5,5-tetramethyl-1,3,2-dioxaborolan-2-yl)-1Hindole $(25.7 \mathrm{mg}, 0.100 \mathrm{mmol})$ was reacted with phenylacetylene $(30.6 \mathrm{mg}, 33 \mu \mathrm{L}, 0.3 \mathrm{mmol})$. After the reaction was complete, volatiles were removed under reduced pressure. ${ }^{1} \mathrm{H}$ NMR analysis of the crude mixture using dimethylsulfone as an internal standard showed formation of product $4 \mathrm{n}$ in an $84 \%$ yield. Attempts to purify $4 \mathrm{n}$ proved unsuccessful due to its instability on silica.

\section{3-(1-Phenylvinyl)-1H-indole (40) ${ }^{9}$}<smiles>C=C(c1ccccc1)c1c[nH]c2ccccc12</smiles>

Following general procedure C, 2-(4,4,5,5-tetramethyl-1,3,2-dioxaborolan-2-yl)- $1 \mathrm{H}$-indole ( $24.3 \mathrm{mg}, 0.100 \mathrm{mmol}$ ) was reacted with phenylacetylene $(30.6 \mathrm{mg}, 33 \mu \mathrm{L}, 0.3 \mathrm{mmol}$ ). After the reaction was complete, volatiles were removed under reduced pressure. The resulting residue was then dissolved in dichloromethane $(1 \mathrm{~mL})$ and silica gel was added until it formed a slurry. The resulting slurry was then stirred at room temperature for $4 \mathrm{~h}$. The slurry was then diluted with more dichloromethane and filtered, before the filter cake was washed several times with dichloromethane. The organics were then combined and concentrated under reduced pressure to yield the crude product. The crude product was purified by silica gel flash column chromatography $(12: 1 \rightarrow 10: 1$ hexane/EtOAc) to yield product 40 (12.0 mg, 0.055 $\mathrm{mmol}, 58 \%)$ as a brown oil.

$\mathbf{R}_{\mathbf{f}} 0.27$ (10:1 hexane/EtOAc); $\mathbf{v}_{\max } / \mathrm{cm}^{-1} 3416(\mathrm{~N}-\mathrm{H}), 3076$ (Csp $\left.{ }^{2}-\mathrm{H}\right), 3054$ (Csp $\left.{ }^{2}-\mathrm{H}\right), 3027$ (Csp ${ }^{2}-$ H), 1635 (C=C), 1599 (C=C Ar), 1492 (C=C Ar), 1571 (C=C Ar), 1491 (C=C Ar), 1456 (C=C Ar); ${ }^{1} \mathrm{H}$ NMR $\left(\mathrm{CDCl}_{3}, 300 \mathrm{MHz}\right) \delta_{\mathrm{H}} 8.15$ (br s, 1H, N-H), $7.56-7.51(\mathrm{~m}, 1 \mathrm{H}, \mathrm{Ar}-\mathrm{H}), 7.51-7.45(\mathrm{~m}, 2 \mathrm{H}$, Ar-H), 7.40 (ddd, $J=8.1,1.0,1.0 \mathrm{~Hz}, 1 \mathrm{H}, \mathrm{Ar}-\mathrm{H}), 7.37-7.31(\mathrm{~m}, 3 \mathrm{H}, \mathrm{Ar}-\mathrm{H}), 7.22$ (ddd, $J=8.1$, 7.0, $1.0 \mathrm{~Hz}, 1 \mathrm{H}, \mathrm{Ar}-\mathrm{H}$ ), 7.14 (d, J = 2.6 Hz, 1H, Ar-H), 7.10 (ddd, J = 8.1, 7.0, $1.0 \mathrm{~Hz}, 1 \mathrm{H}, \mathrm{Ar}-\mathrm{H}$ ), $5.60(\mathrm{~d}, J=1.7 \mathrm{~Hz}, 1 \mathrm{H}, \mathrm{C}=\mathrm{CHH}), 5.44(\mathrm{~d}, J=1.7 \mathrm{~Hz}, 1 \mathrm{H}, \mathrm{C}=\mathrm{CHH}) ;{ }^{13} \mathrm{C} \mathrm{NMR}\left(\mathrm{CDCl}_{3}, 75 \mathrm{MHz}\right) \delta_{\mathrm{C}}$ $143.2(\mathrm{C}), 142.4(\mathrm{C}), 136.5(\mathrm{C}), 128.1(\mathrm{CH}), 128.1(\mathrm{CH}), 127.6(\mathrm{CH}), 126.3(\mathrm{C}), 124.3(\mathrm{CH}), 122.3$ $(\mathrm{CH}), 120.7(\mathrm{CH}), 120.1(\mathrm{CH}), 118.1(\mathrm{C}), 112.2\left(\mathrm{CH}_{2}\right), 111.2(\mathrm{CH})$. 
<smiles>C=C(c1ccccc1)c1cn(C)c2ccccc12</smiles>

Following general procedure C, 1-methyl-2-(4,4,5,5-tetramethyl-1,3,2-dioxaborolan-2-yl)-1Hindole $(25.7 \mathrm{mg}, 0.100 \mathrm{mmol})$ was reacted with phenylacetylene $(30.6 \mathrm{mg}, 33 \mu \mathrm{L}, 0.3 \mathrm{mmol})$. After the reaction was complete, volatiles were removed under reduced pressure. The resulting residue was then dissolved in dichloromethane $(1 \mathrm{~mL})$ and silica gel was added until it formed a slurry. The resulting slurry was then stirred at room temperature for $4 \mathrm{~h}$. The slurry was then diluted with more dichloromethane and filtered, before the filter cake was washed several times with dichloromethane. The organics were then combined and concentrated under reduced pressure to yield the crude product. The crude product was purified by silica gel flash column chromatography (50:1 hexane/EtOAc) to yield product $4 \mathrm{p}$ (14.9 mg, $0.064 \mathrm{mmol}$, $64 \%)$ as a yellow oil.

$\mathbf{R}_{\mathbf{f}} 0.27$ (40:1 hexane/EtOAc); $\boldsymbol{v}_{\max } / \mathrm{cm}^{-1} 3053$ (Csp $\left.{ }^{2}-\mathrm{H}\right), 3024$ (Csp $\left.{ }^{2}-\mathrm{H}\right), 2929\left(\mathrm{Csp}^{3}-\mathrm{H}\right), 1612$ $(\mathrm{C}=\mathrm{C}), 1598$ ( $\mathrm{C}=\mathrm{C} \mathrm{Ar}), 1525$ ( $\mathrm{C}=\mathrm{C} \mathrm{Ar}), 1490$ ( $\mathrm{C}=\mathrm{C} \mathrm{Ar}), 1468$ ( $\mathrm{C}=\mathrm{C} \mathrm{Ar}), 1444$ (C=C Ar); ${ }^{1} \mathrm{H}$ NMR $\left(\mathrm{CDCl}_{3}, 400 \mathrm{MHz}\right) \delta_{\mathrm{H}} 7.57$ (ddd, J=8.0, 1.0, $\left.1.0 \mathrm{~Hz}, 1 \mathrm{H}, \mathrm{Ar}-\mathrm{H}\right), 7.51-7.47$ (m, 2H, Ar-H), $7.39-$ $7.32(\mathrm{~m}, 5 \mathrm{H}, \mathrm{Ar}-\mathrm{H}), 7.11$ (ddd, J = 8.0, 7.0, $1.0 \mathrm{~Hz}, 1 \mathrm{H}, \mathrm{Ar}-\mathrm{H}), 6.98(\mathrm{~s}, 1 \mathrm{H}, \mathrm{Ar}-\mathrm{H}), 5.58$ (d, J = 1.6 $\mathrm{Hz}, 1 \mathrm{H}, \mathrm{C}=\mathrm{CHH}), 5.39(\mathrm{~d}, J=1.6 \mathrm{~Hz}, 1 \mathrm{H}, \mathrm{C}=\mathrm{CHH}), 3.78\left(\mathrm{~s}, 3 \mathrm{H}, \mathrm{CH}_{3}\right) ;{ }^{13} \mathrm{C} \mathrm{NMR}\left(\mathrm{CDCl}_{3}, 101 \mathrm{MHz}\right)$ $\delta_{\mathrm{C}} 143.4(\mathrm{C}), 142.8(\mathrm{C}), 137.5(\mathrm{C}), 129.1(\mathrm{CH}), 128.2(\mathrm{CH}), 128.1(\mathrm{CH}), 127.5(\mathrm{CH}), 126.8(\mathrm{C})$, $121.9(\mathrm{CH}), 120.9(\mathrm{CH}), 119.7(\mathrm{CH}), 116.6(\mathrm{C}), 111.5\left(\mathrm{CH}_{2}\right), 109.4(\mathrm{CH}), 32.8\left(\mathrm{CH}_{3}\right)$.

\section{5-Methoxy-3-(1-(4-methoxyphenyl)vinyl)-2-methyl-1H-indole (4q)}<smiles>C=C(c1ccc(OC)cc1)c1c(C)[nH]c2ccc(OC)cc12</smiles>

Following general procedure A, 2,5-dimethoxy- $1 H$-indole $(16.1 \mathrm{mg}, 0.100 \mathrm{mmol})$ was reacted with 4-ethynylanisole $(39.6 \mathrm{mg}, 39 \mu \mathrm{L}, 0.3 \mathrm{mmol})$. The crude mixture was purified by silica gel flash column chromatography (5:1 hexane/EtOAc) to yield product $4 \mathbf{q}(27.4 \mathrm{mg}, 0.094 \mathrm{mmol}$, 94\%) as an brown oil.

$\mathbf{R}_{\mathbf{f}} 0.18$ (7:1 hexane/EtOAC); $\mathbf{v}_{\max } / \mathrm{cm}^{-1} 3397(\mathrm{~N}-\mathrm{H}), 3068$ (Csp $\left.{ }^{2}-\mathrm{H}\right), 3001\left(\mathrm{Csp}^{2}-\mathrm{H}\right) 2935$ (Csp ${ }^{3}-$ $\mathrm{H}), 2834$ ( $\left.\mathrm{Csp}^{3}-\mathrm{H}\right), 1604$ ( $\left.\mathrm{C}=\mathrm{C}\right), 1508$ (C=C Ar), 1483 ( $\left.\mathrm{C}=\mathrm{C} \mathrm{Ar}\right), 1463$ (C=C Ar), 1450 (C=C Ar), 1240 (C-O-Ar), 1169 (C-O-C); ${ }^{1} \mathrm{H}$ NMR (CDCl, 400 MHz) $\delta_{H} 7.82$ (br s, $\left.1 \mathrm{H}, \mathrm{N}-\mathrm{H}\right), 7.35$ (d, J = 8.9 $\mathrm{Hz}, 2 \mathrm{H}, \mathrm{Ar}-\mathrm{H}$ ), 7.18 (d, J = 8.7 Hz, 1H, Ar-H), 6.84 (d, J = 8.9 Hz, 2H, Ar-H), 6.78 (dd, J = 8.7, 2.5 $\mathrm{Hz}, 1 \mathrm{H}, \mathrm{Ar}-\mathrm{H}), 6.72(\mathrm{~d}, J=2.5 \mathrm{~Hz}, 1 \mathrm{H}, \mathrm{Ar}-\mathrm{H}), 5.66(\mathrm{~d}, J=1.9 \mathrm{~Hz}, 1 \mathrm{H}, \mathrm{C}=\mathrm{CHH}), 5.23(\mathrm{~d}, J=1.9 \mathrm{~Hz}$, 
$1 \mathrm{H}, \mathrm{C}=\mathrm{CHH}), 3.82\left(\mathrm{~s}, 3 \mathrm{H}, \mathrm{OCH}_{3}\right), 3.71\left(\mathrm{~s}, 3 \mathrm{H}, \mathrm{OCH}_{3}\right), 2.26\left(\mathrm{~s}, 3 \mathrm{H}, \mathrm{OCH}_{3}\right) ;{ }^{13} \mathrm{C} \mathrm{NMR}\left(\mathrm{CDCl}_{3}, 101\right.$ $\mathrm{MHz}$ ) $\delta_{\mathrm{c}} 159.3$ (C), 154.2 (C), 141.9 (C), 134.4 (C), 133.8 (C), 130.3 (C), 129.1 (C), $128.5(\mathrm{CH})$, $114.3(\mathrm{C}), 113.6(\mathrm{CH}), 113.0\left(\mathrm{CH}_{2}\right), 110.9(\mathrm{CH}), 110.7(\mathrm{CH}), 102.3(\mathrm{CH}), 55.8\left(\mathrm{CH}_{3}\right), 55.3\left(\mathrm{CH}_{3}\right)$, $12.9\left(\mathrm{CH}_{3}\right)$; HRMS (TOF MS ASAP+) m/z: $[\mathrm{M}+\mathrm{H}]^{+}$Calcd for $\mathrm{C}_{19} \mathrm{H}_{20} \mathrm{NO}_{2}$ 294.1494; Found 294.1495.

\section{3-(1-(4-Nitrophenyl)vinyl)-2-phenyl-1H-indole (4r)}<smiles>C=C(c1ccc([N+](=O)[O-])cc1)c1c(-c2ccccc2)[nH]c2ccccc12</smiles>

Following general procedure A, 2-phenylindole $(19.3 \mathrm{mg}, 0.100 \mathrm{mmol})$ was reacted with 1ethynyl-4-nitrobenzene ( $44.1 \mathrm{mg}, 0.3 \mathrm{mmol}$ ). The crude mixture was purified by silica gel flash column chromatography $(20: 1 \rightarrow 15: 1 \rightarrow 10: 1$ hexane/EtOAc) to yield product $4 \mathrm{r}(24.2 \mathrm{mg}$, $0.071 \mathrm{mmol}, 71 \%$ ) as an orange solid.

$\mathbf{R}_{\mathbf{f}} 0.20$ (10:1 hexane/EtOAc); $\boldsymbol{v}_{\max } / \mathrm{cm}^{-1} 3405$ (N-H), 3059 (Csp $\left.{ }^{2}-\mathrm{H}\right) 3023$ (Csp $\left.{ }^{2}-\mathrm{H}\right), 1593$ (C=C), $1513\left(\mathrm{NO}_{2}\right), 1487$ (C=C Ar), 1456 (C=C Ar), 1449 (C=C Ar), $1341\left(\mathrm{NO}_{2}\right) ;{ }^{1} \mathrm{H}$ NMR $\left(\mathrm{CDCl}_{3}, 400\right.$ $\mathrm{MHz}) \delta_{\mathrm{H}} 8.33(\mathrm{~s}, 1 \mathrm{H}, \mathrm{N}-\mathrm{H}), 8.04(\mathrm{~d}, J=9.1 \mathrm{~Hz}, 2 \mathrm{H}, \mathrm{Ar}-\mathrm{H}), 7.53$ (d, J = $\left.9.1 \mathrm{~Hz}, 2 \mathrm{H}, \operatorname{Ar}-\mathrm{H}\right), 7.49-$ $7.44(\mathrm{~m}, 3 \mathrm{H}, \mathrm{Ar}-\mathrm{H}), 7.39(\mathrm{dm}, J=8.1,1 \mathrm{H}, \mathrm{Ar}-\mathrm{H}), 7.31-7.21(\mathrm{~m}, 4 \mathrm{H}, \mathrm{Ar}-\mathrm{H}), 7.12$ (ddd, $J=8.1$, 7.1, $0.9 \mathrm{~Hz}, 1 \mathrm{H}, \mathrm{Ar}-\mathrm{H}), 5.98(\mathrm{~d}, J=1.2 \mathrm{~Hz}, 1 \mathrm{H}, \mathrm{C}=\mathrm{CHH}), 5.64(\mathrm{~d}, J=1.2 \mathrm{~Hz}, 1 \mathrm{H} \mathrm{C}=\mathrm{CHH}) ;{ }^{13} \mathrm{C} \mathrm{NMR}$ $\left(\mathrm{CDCl}_{3}, 101 \mathrm{MHz}\right) \delta_{\mathrm{C}} 147.8$ (C), 147.1 (C), 140.8 (C), 135.9 (C), 135.7 (C), 132.2 (C), 129.0 (C), $128.7(\mathrm{CH}), 128.0(\mathrm{CH}), 127.7(\mathrm{CH}), 127.6(\mathrm{CH}), 123.4(\mathrm{CH}), 122.9(\mathrm{CH}), 120.6(\mathrm{CH}), 120.3\left(\mathrm{CH}_{2}\right)$, $119.8(\mathrm{CH}), 113.3(\mathrm{C}), 111.0(\mathrm{CH}) ;$ m.p. $=164-166^{\circ} \mathrm{C}$; HRMS (FTMS + p NSI) m/z: $[\mathrm{M}+\mathrm{H}]^{+} \mathrm{Calcd}$ for $\mathrm{C}_{22} \mathrm{H}_{17} \mathrm{O}_{2} \mathrm{~N}_{2}$ 341.1285; Found 341.1288.

\section{3-(1-(4-Chlorophenyl)vinyl)-2-phenyl-1H-indole (4s)}<smiles>C=C(c1ccc(Cl)cc1)c1c(-c2ccccc2)[nH]c2ccccc12</smiles>

Following general procedure A, 2-phenylindole $(19.3 \mathrm{mg}, 0.100 \mathrm{mmol}$ ) was reacted with 1chloro-4-ethynylbenzene $(41.0 \mathrm{mg}, 0.3 \mathrm{mmol})$. The crude mixture was purified by silica gel flash column chromatography (10:1 hexane/EtOAc) to yield product $4 \mathbf{s}(33.0 \mathrm{mg}, 0.100 \mathrm{mmol}$, quant.) as a yellow solid.

$\mathbf{R}_{\mathbf{f}} 0.35$ (10:1 hexane/EtOAc); $\mathbf{v}_{\max } / \mathrm{cm}^{-1} 3420(\mathrm{~N}-\mathrm{H}), 3059$ (Csp $\left.{ }^{2}-\mathrm{H}\right), 3017$ (Csp $\left.{ }^{2}-\mathrm{H}\right), 1604$ (C=C), 1487 (C=C Ar), 1456 (C=C Ar), 1448 (C=C Ar); ${ }^{1} \mathrm{H} \mathrm{NMR}\left(\mathrm{CDCl}_{3}, 400 \mathrm{MHz}\right) \delta_{\mathrm{H}} 8.24$ (br s, $1 \mathrm{H}, \mathrm{N}-$ H), $7.55-7.52(\mathrm{~m}, 2 \mathrm{H}, \mathrm{Ar}-\mathrm{H}), 7.43$ (ddd, J = 8.1, 0.9, 0.9 Hz, 1H, Ar-H), $7.40-7.30(\mathrm{~m}, 5 \mathrm{H}, \mathrm{Ar}-$ 
H), $7.29-7.22(\mathrm{~m}, 2 \mathrm{H}, \mathrm{Ar}-\mathrm{H}), 7.22-7.17(\mathrm{~m}, 2 \mathrm{H}, \mathrm{Ar}-\mathrm{H}), 7.10$ (ddd, J=8.1, 7.1, 0.9 Hz, 1H, Ar$\mathrm{H}), 5.84(\mathrm{~d}, J=1.5 \mathrm{~Hz}, 1 \mathrm{H}, \mathrm{C}=\mathrm{CHH}), 5.41(\mathrm{~d}, J=1.5 \mathrm{~Hz}, 1 \mathrm{H}, \mathrm{C}=\mathrm{CHH}) . ;{ }^{13} \mathrm{C} \mathrm{NMR}\left(\mathrm{CDCl}_{3}, 101 \mathrm{MHz}\right)$ 反c 141.1 (C), 139.5 (C), 135.8 (C), 135.3 (C), 133.3 (C), 132.4 (C), 129.3 (C), 128.6 (CH), 128.3 $(\mathrm{CH}), 128.2(\mathrm{CH}), 127.7(\mathrm{CH}), 127.6(\mathrm{CH}), 122.7(\mathrm{CH}), 120.3(\mathrm{CH}), 120.1(\mathrm{CH}), 117.2\left(\mathrm{CH}_{2}\right), 114.2$ (C), 110.8 (CH); m.p. = $129-131{ }^{\circ} \mathrm{C}$; HRMS (FTMS + c ESI) m/z: $[\mathrm{M}+\mathrm{H}]^{+}$Calcd for $\mathrm{C}_{22} \mathrm{H}_{17} \mathrm{NCl}$ 330.1044; Found 330.1040.

\section{3-(1-(3-Chlorophenyl)vinyl)-2-phenyl-1H-indole (4t)}<smiles>C=C(c1cccc(Cl)c1)c1c(-c2ccccc2)[nH]c2ccccc12</smiles>

Following general procedure A, 2-phenylindole $(19.3 \mathrm{mg}, 0.100 \mathrm{mmol})$ was reacted with 1chloro-3-ethynylbenzene $(41.0 \mathrm{mg}, 37 \mu \mathrm{L}, 0.3 \mathrm{mmol})$. The crude mixture was purified by silica gel flash column chromatography (10:1 hexane/EtOAc) to yield product $4 \mathbf{t}(33.0 \mathrm{mg}, 0.100$ mmol, quant.) as a yellow oil.

$\mathbf{R}_{\mathbf{f}} 0.23$ (10:1 hexane/EtOAc); $\boldsymbol{v}_{\max } / \mathrm{cm}^{-1} 3419(\mathrm{~N}-\mathrm{H}), 3059\left(\mathrm{Csp}^{2}-\mathrm{H}\right), 3013\left(\mathrm{Csp}^{2}-\mathrm{H}\right), 1603$ (C=C

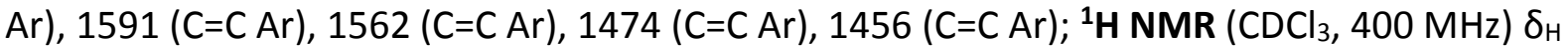
8.25 (br s, $1 \mathrm{H}, \mathrm{Ar}-\mathrm{H}), 7.55-7.51(\mathrm{~m}, 2 \mathrm{H}, \mathrm{Ar}-\mathrm{H}), 7.45-7.41(\mathrm{~m}, 2 \mathrm{H}, \mathrm{Ar}-\mathrm{H}), 7.39(\mathrm{dm}, J=8.1 \mathrm{~Hz}$, $1 \mathrm{H}, \mathrm{Ar}-\mathrm{H}$ ), $7.35-7.26(\mathrm{~m}, 4 \mathrm{H}, \mathrm{Ar}-\mathrm{H}), 7.25-7.23$ (m, $1 \mathrm{H}, \mathrm{Ar}-\mathrm{H}), 7.18$ (ddd, $J=8.1,2.0,1.4 \mathrm{~Hz}$, $1 \mathrm{H}, \mathrm{Ar}-\mathrm{H}), 7.16-7.13(\mathrm{~m}, 1 \mathrm{H}, \mathrm{Ar}-\mathrm{H}), 7.11$ (ddd, $J=8.1,7.1,1.0 \mathrm{~Hz}, 1 \mathrm{H}, \mathrm{Ar}-\mathrm{H}), 5.86(\mathrm{~d}, J=1.4$ $\mathrm{Hz}, 1 \mathrm{H}, \mathrm{C}=\mathrm{CHH}), 5.45$ (d, J = 1.4 Hz, 1H, C=CHH); ${ }^{13} \mathrm{C} \mathrm{NMR}\left(\mathrm{CDCl}_{3}, 101 \mathrm{MHz}\right) \delta_{\mathrm{C}} 143.0(\mathrm{C}), 141.1$ (C), 135.8 (C), 135.4 (C), 134.1 (C), 132.4 (C), $129.3(\mathrm{CH}), 129.2(\mathrm{C}), 128.5(\mathrm{CH}), 127.8(\mathrm{CH})$, 127.7 (CH), $127.5(\mathrm{CH}), 127.0(\mathrm{CH}), 125.3(\mathrm{CH}), 122.7(\mathrm{CH}), 120.4(\mathrm{CH}), 120.1(\mathrm{CH}), 117.8\left(\mathrm{CH}_{2}\right)$, 113.9 (C), $110.8(\mathrm{CH})$; HRMS (FTMS + c ESI) m/z: [M + H] ${ }^{+}$Calcd for $\mathrm{C}_{22} \mathrm{H}_{17} \mathrm{NCl} 330.1044$; Found 330.1040 .

\section{3-(1-(2-Chlorophenyl)vinyl)-2-phenyl-1H-indole (4u)}<smiles>C=C(c1ccccc1Cl)c1c(-c2ccccc2)[nH]c2ccccc12</smiles>

Following general procedure B, 2-phenylindole $(19.3 \mathrm{mg}, 0.100 \mathrm{mmol})$ was reacted with 1chloro-2-ethynylbenzene $(41.0 \mathrm{mg}, 36 \mu \mathrm{L}, 0.3 \mathrm{mmol})$. The crude mixture was purified by silica gel flash column chromatography (10:1 hexane/EtOAc) to yield product $4 \mathbf{u}(31.7 \mathrm{mg}, 0.096$ mmol, 96\%) as a yellow solid. 
$\mathbf{R}_{\mathbf{f}} 0.26$ (10:1 hexane/EtOAc); $\boldsymbol{v}_{\max } / \mathrm{cm}^{-1} 3419(\mathrm{~N}-\mathrm{H}), 3059$ (Csp $\left.{ }^{2}-\mathrm{H}\right), 3039\left(\mathrm{Csp}^{2}-\mathrm{H}\right), 1603(\mathrm{C}=\mathrm{C})$, 1485 ( $C=C$ Ar), 1472 (C=C Ar) 1455 (C=C Ar), 1447 (C=C Ar), 1434 (C=C Ar); ${ }^{1} \mathbf{H}$ NMR $\left(C^{2} C_{3}, 400\right.$ $\mathrm{MHz}) \delta_{\mathrm{H}} 8.11$ (br s, 1H, N-H), $7.59-7.55(\mathrm{~m}, 2 \mathrm{H}, \mathrm{Ar}-\mathrm{H}), 7.43(\mathrm{dm}, J=8.1 \mathrm{~Hz}, 1 \mathrm{H}, \operatorname{Ar}-\mathrm{H}), 7.39$ (ddd, $J=8.1,0.9,0.9 \mathrm{~Hz}, 1 \mathrm{H}, \mathrm{Ar}-\mathrm{H}$ ), $7.33-7.27$ (m, 3H, Ar-H), 7.26- 7.19 (m, 3H, Ar-H), 7.12 $-7.05(\mathrm{~m}, 3 \mathrm{H}, \mathrm{Ar}-\mathrm{H}), 5.72(\mathrm{~d}, J=1.7 \mathrm{~Hz}, 1 \mathrm{H}, \mathrm{C}=\mathrm{CHH}), 5.63(\mathrm{~d}, J=1.7 \mathrm{~Hz}, 1 \mathrm{H}, \mathrm{C}=\mathrm{CHH}) ;{ }^{13} \mathrm{C} \mathrm{NMR}$ $\left(\mathrm{CDCl}_{3}, 101 \mathrm{MHz}\right) \delta_{\mathrm{c}} 140.9$ (C), 140.1 (C), 135.8 (C), 135.3 (C), 132.8 (C), 132.8 (C), $131.4(\mathrm{CH})$, $129.8(\mathrm{CH}), 128.7(\mathrm{C}), 128.4(\mathrm{CH}), 128.2(\mathrm{CH}), 128.2(\mathrm{CH}), 127.7(\mathrm{CH}), 126.2(\mathrm{CH}), 122.4(\mathrm{CH})$, $120.3\left(\mathrm{CH}_{2}\right), 120.3(\mathrm{CH}), 120.1(\mathrm{CH}), 114.8(\mathrm{C}), 110.8(\mathrm{CH}) ;$ m.p. $=65-68^{\circ} \mathrm{C}$; HRMS (FTMS + C ESI) $\mathrm{m} / \mathrm{z}:[\mathrm{M}+\mathrm{H}]^{+}$Calcd for $\mathrm{C}_{22} \mathrm{H}_{17} \mathrm{NCl} 330.1044$; Found 330.1039 .

(Z)-2-Phenyl-3-(1-phenylprop-1-en-1-yl)-1H-indole (4v)<smiles>C/C=C(\c1ccccc1)c1c(-c2ccccc2)[nH]c2ccccc12</smiles>

Following general procedure $\mathrm{E}, 2$-phenylindole $(19.3 \mathrm{mg}, 0.100 \mathrm{mmol})$ was reacted with prop1-yn-1-ylbenzene ( $34.8 \mathrm{mg}, 40 \mu \mathrm{L}, 0.3 \mathrm{mmol}$ ). The crude mixture was purified by silica gel flash column chromatography $(20: 1 \rightarrow 15: 1$ hexane/EtOAc) to yield product $4 \mathbf{v}(29.0 \mathrm{mg}, 0.094$ $\mathrm{mmol}, 94 \%)$ as a yellow oil.

$\mathbf{R}_{\mathbf{f}} 0.24$ (10:1 hexane/EtOAc); $\mathbf{v}_{\max } / \mathrm{cm}^{-1} 3415(\mathrm{~N}-\mathrm{H}), 3056$ (Csp $\left.{ }^{2}-\mathrm{H}\right), 3026$ (Csp $\left.{ }^{2}-\mathrm{H}\right), 2905$ (Csp ${ }^{3}-$ H), 1602 (C=C), 1573 (C=C Ar), 1489 (C=C Ar), 1456 (C=C Ar), 1447 (C=C Ar); ${ }^{1}$ H NMR (CDCl $300 \mathrm{MHz}$ ) $\delta_{\mathrm{H}} 8.32$ (br s, $\left.1 \mathrm{H}, \mathrm{N}-\mathrm{H}\right), 7.65-7.58(\mathrm{~m}, 2 \mathrm{H}, \mathrm{Ar}-\mathrm{H}), 7.46-7.38(\mathrm{~m}, 3 \mathrm{H}, \mathrm{Ar}-\mathrm{H}), 7.37-$ $7.31(\mathrm{~m}, 2 \mathrm{H}, \mathrm{Ar}-\mathrm{H}), 7.29-7.17(\mathrm{~m}, 6 \mathrm{H}, \mathrm{Ar}-\mathrm{H}), 7.08-7.02(\mathrm{~m}, 1 \mathrm{H}, \mathrm{Ar}-\mathrm{H}), 6.40(\mathrm{q}, J=6.9 \mathrm{~Hz}, 1 \mathrm{H}$, $\mathrm{C}=\mathrm{C}-\mathrm{H}), 1.52\left(\mathrm{~d}, J=6.9 \mathrm{~Hz}, 3 \mathrm{H}, \mathrm{CH}_{3}\right) . ;{ }^{13} \mathrm{C} \mathrm{NMR}\left(\mathrm{CDCl}_{3}, 75 \mathrm{MHz}\right) \delta_{\mathrm{c}} 142.1$ (C), 136.0 (C), 134.8 (C), $134.8(\mathrm{C}), 133.0(\mathrm{C}), 129.5(\mathrm{C}), 128.7(\mathrm{CH}), 128.2(\mathrm{CH}), 127.5(\mathrm{CH}), 126.7(\mathrm{CH}), 126.6(\mathrm{CH})$, $126.4(\mathrm{CH}), 126.4(\mathrm{CH}), 122.5(\mathrm{CH}), 120.4(\mathrm{CH}), 120.0(\mathrm{CH}), 112.1(\mathrm{C}), 110.7(\mathrm{CH}), 15.8\left(\mathrm{CH}_{3}\right)$; HRMS (FTMS + p NSI) m/z: [M + H] $]^{+}$Calcd for $\mathrm{C}_{23} \mathrm{H}_{20} \mathrm{~N}$ 310.1590; Found 310.1593.

Stereochemistry of the major isomer was determined by NOESY:

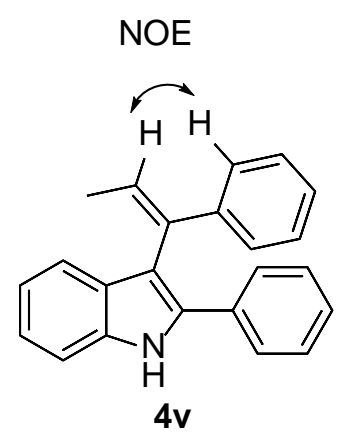




\section{(Z)-2-Phenyl-3-(1-phenylhex-1-en-1-yl)-1H-indole (4w)}<smiles>CCCC/C=C(\c1ccccc1)c1c(-c2ccccc2)[nH]c2ccccc12</smiles>

Following general procedure $\mathrm{E}, 2$-phenylindole $(19.3 \mathrm{mg}, 0.100 \mathrm{mmol})$ was reacted with hex1-yn-1-ylbenzene ( $47.5 \mathrm{mg}, 53 \mu \mathrm{L}, 0.3 \mathrm{mmol}$ ). The crude mixture was purified by silica gel flash column chromatography (20:1 hexane/EtOAc) to yield product $4 \mathbf{w}(33.8 \mathrm{mg}, 0.096 \mathrm{mmol}$, 96\%) as a brown oil.

$\mathbf{R}_{\mathbf{f}} 0.20$ (10:1 hexane/EtOAc); $\mathbf{v}_{\max } / \mathrm{cm}^{-1} 3413(\mathrm{~N}-\mathrm{H}), 3056$ (Csp $\left.{ }^{2}-\mathrm{H}\right), 3025$ (Csp $\left.{ }^{2}-\mathrm{H}\right), 2956$ (Csp ${ }^{3}-$ H), 2927 ( $\left.\mathrm{Csp}^{3}-\mathrm{H}\right), 1603$ (C=C), 1573 (C=C Ar), 1489 (C=C Ar), 1455 (C=C Ar), 1447 (C=C Ar); ${ }^{1} \mathrm{H}$ NMR $\left(\mathrm{CDCl}_{3}, 400 \mathrm{MHz}\right) \delta_{\mathrm{H}} 8.30($ br s, $1 \mathrm{H}, \mathrm{N}-\mathrm{H}), 7.64-7.60(\mathrm{~m}, 2 \mathrm{H}, \mathrm{Ar}-\mathrm{H}), 7.45-7.40(\mathrm{~m}, 3 \mathrm{H}$, Ar-H), $7.38-7.29(\mathrm{~m}, 2 \mathrm{H}, \mathrm{Ar}-\mathrm{H}), 7.29-7.18(\mathrm{~m}, 6 \mathrm{H}, \mathrm{Ar}-\mathrm{H}), 7.05$ (ddd, J = 8.0, 7.0, $1.0 \mathrm{~Hz}, 1 \mathrm{H}$, $\operatorname{Ar}-\mathrm{H}), 6.34(\mathrm{t}, J=7.2 \mathrm{~Hz}, 1 \mathrm{H}, \mathrm{C}=\mathrm{C}-\mathrm{H}), 2.04-1.75\left(\mathrm{~m}, 2 \mathrm{H}, \mathrm{CH}_{2}\right), 1.40-1.07\left(\mathrm{~m}, 4 \mathrm{H}, \mathrm{CH}_{2}\right), 0.71$ $\left(\mathrm{t}, J=7.0 \mathrm{~Hz}, 3 \mathrm{H}, \mathrm{CH}_{3}\right) . ;{ }^{13} \mathrm{C} \mathrm{NMR}\left(\mathrm{CDCl}_{3}, 101 \mathrm{MHz}\right) \delta_{\mathrm{c}} 142.1$ (C), 136.0 (C), 134.6 (C), 133.4 $(\mathrm{CH}), 132.9(\mathrm{C}), 132.9(\mathrm{C}), 129.7(\mathrm{C}), 128.7(\mathrm{CH}), 128.2(\mathrm{CH}), 127.5(\mathrm{CH}), 126.6(\mathrm{CH}), 126.6(\mathrm{CH})$, $126.5(\mathrm{CH}), 122.4(\mathrm{CH}), 120.3(\mathrm{CH}), 120.0(\mathrm{CH}), 112.6(\mathrm{C}), 110.6(\mathrm{CH}), 31.3\left(\mathrm{CH}_{2}\right), 29.8\left(\mathrm{CH}_{2}\right)$, $22.4\left(\mathrm{CH}_{2}\right), 13.9\left(\mathrm{CH}_{3}\right)$; HRMS (TOF MS ASAP+) m/z: $[\mathrm{M}+\mathrm{H}]^{+}$Calcd for $\mathrm{C}_{26} \mathrm{H}_{26} \mathrm{~N} 352.2065$; Found 352.2062 .

Stereochemistry of the major isomer was determined by NOESY:

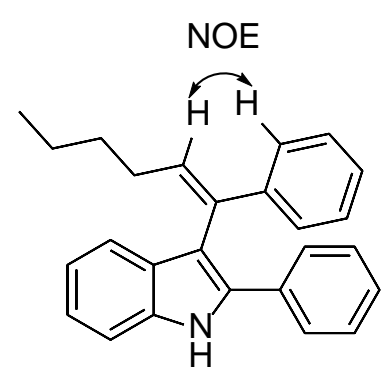

3-(1-Cyclohexylvinyl)-2-phenyl-1H-indole (4x) and (Z)-3-(2-Cyclohexylvinyl)-2-phenyl-1Hindole (4x')<smiles>C=C(c1c(-c2ccccc2)[nH]c2ccccc12)C1CCCCC1</smiles>

Following general procedure $D, 2$-phenylindole $(19.3 \mathrm{mg}, 0.100 \mathrm{mmol})$ was reacted with cyclohexylacetylene $(32.5 \mathrm{mg}, 39 \mu \mathrm{L}, 0.3 \mathrm{mmol})$. The crude mixture was purified by silica gel 
flash column chromatography (20:1 hexane/EtOAC) to yield products $4 \mathbf{x}$ and $\mathbf{4} \mathbf{x}^{\prime}$ as a 9:1 mixture of inseparable isomers $(27.2 \mathrm{mg}, 0.090 \mathrm{mmol}, 90 \%)$ as a purple solid.

$\mathbf{R}_{\mathbf{f}} 0.21$ (20:1 hexane/EtOAc); $\boldsymbol{v}_{\max } / \mathrm{cm}^{-1} 3386(\mathrm{~N}-\mathrm{H}), 3081$ (Csp $\left.{ }^{2}-\mathrm{H}\right), 3056$ (Csp $\left.{ }^{2}-\mathrm{H}\right), 2920$ (Csp ${ }^{3}$ $\mathrm{H}$ ), 2850 ( $\mathrm{Csp}^{3}-\mathrm{H}$ ), 1602 (C=C), 1485 (C=C Ar), 1454 (C=C Ar), 1445 (C=C Ar); ${ }^{1} \mathrm{H} \mathrm{NMR} \mathrm{(CDCl}{ }_{3}$, $400 \mathrm{MHz}$ ) Selected signals for major and minor isomers $\delta_{H} 6.35(\mathrm{dd}, J=11.2,0.6 \mathrm{~Hz}, 1 \mathrm{H}, H \mathrm{C}=\mathrm{CH}$ minor), 5.64 (dd, $J=11.2,10.1 \mathrm{~Hz}, 1 \mathrm{H}, \mathrm{HC}=\mathrm{CH}$ minor), 5.38 (dd, $J=2.0,1.5 \mathrm{~Hz}, 1 \mathrm{H}, \mathrm{C}=\mathrm{CHH}$ major), 5.22 (dd, $J=2.0,0.6 \mathrm{~Hz}, 1 \mathrm{H}, \mathrm{C}=\mathrm{CHH}$ major); ${ }^{13} \mathrm{C} \mathrm{NMR}\left(\mathrm{CDCl}_{3}, 101 \mathrm{MHz}\right.$ ) Assignments for major isomer only $\delta_{C} 148.8$ (C), 135.7 (C), 133.4 (C), 132.8 (C), 129.9 (C), 128.6 (CH), 127.5 $(\mathrm{CH}), 127.3(\mathrm{CH}), 122.4(\mathrm{CH}), 120.1(\mathrm{CH}), 120.0(\mathrm{CH}), 117.1(\mathrm{C}), 113.6\left(\mathrm{CH}_{2}\right), 110.6(\mathrm{CH}), 44.6$ (CH), $32.7\left(\mathrm{CH}_{2}\right), 26.7\left(\mathrm{CH}_{2}\right), 26.4\left(\mathrm{CH}_{2}\right) ;$ m.p. = 78-81 ${ }^{\circ} \mathrm{C}$; HRMS (FTMS + c ESI) m/z: $[\mathrm{M}+\mathrm{H}]^{+}$ Calcd for $\mathrm{C}_{22} \mathrm{H}_{24} \mathrm{~N}$ 302.1903; Found 302.1897.

ebmh273char.1.fid

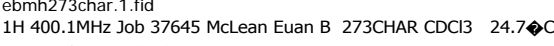
proton characterisation

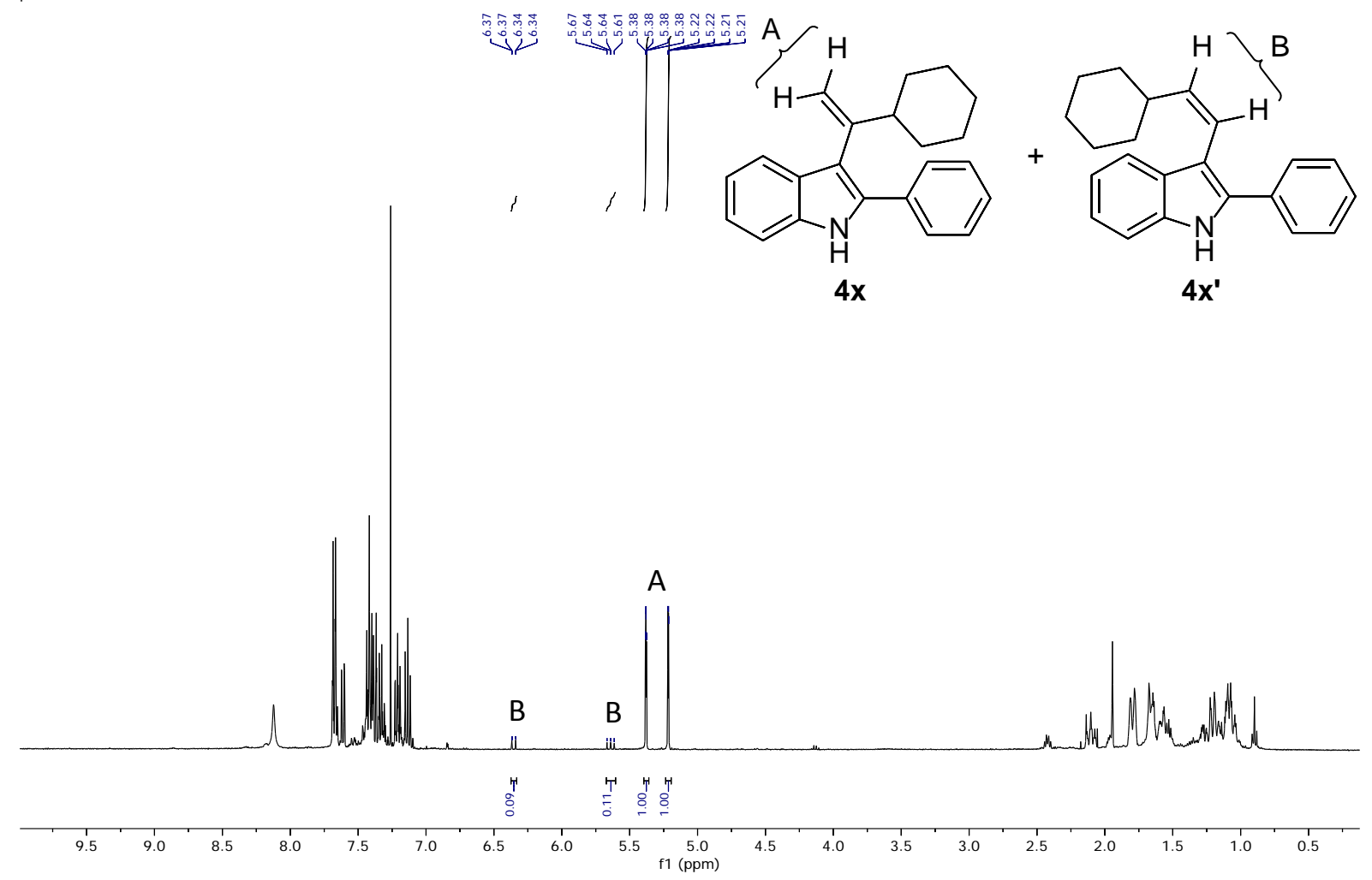



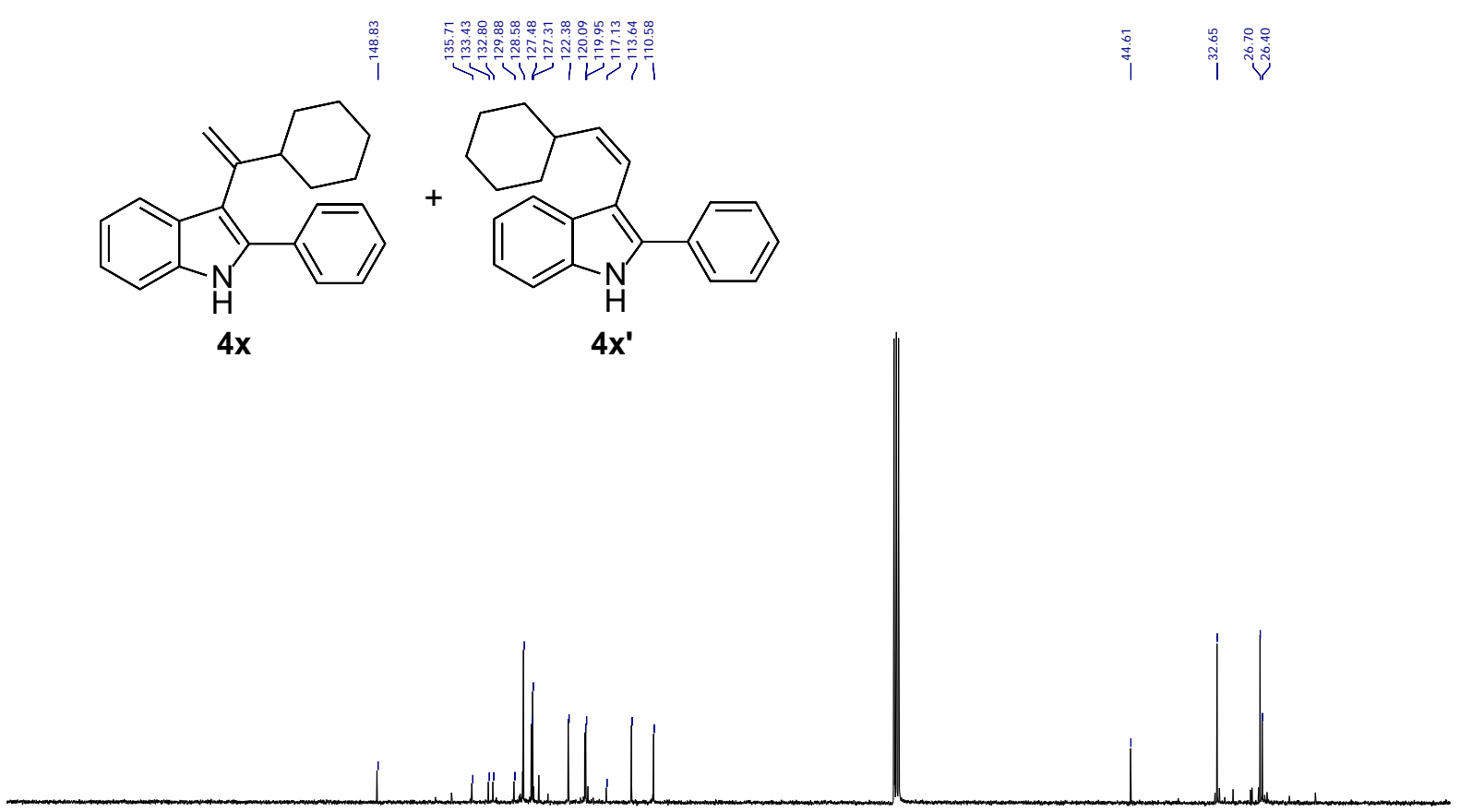$$
30
$$

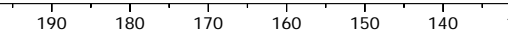

\section{2-Phenyl-3-(1-(thiophen-3-yl)vinyl)-1H-indole (4aa)}<smiles>C=C(c1ccsc1)c1c(-c2ccccc2)[nH]c2ccccc12</smiles>

Following general procedure A, 2-phenylindole $(19.3 \mathrm{mg}, 0.100 \mathrm{mmol})$ was reacted with 3ethynylthiophene $(32.4 \mathrm{mg}, 30 \mu \mathrm{L}, 0.3 \mathrm{mmol})$. The crude mixture was purified by silica gel flash column chromatography (10:1 hexane/EtOAc) to yield product 4 aa $(33.0 \mathrm{mg}, 0.100$ mmol, quant.) as a brown oil.

$\mathbf{R}_{\mathbf{f}} 0.32$ (10:1 hexane/EtOAc); $\mathbf{v}_{\max } / \mathrm{cm}^{-1} 3413(\mathrm{~N}-\mathrm{H}), 3058$ (Csp $\left.{ }^{2}-\mathrm{H}\right), 3007\left(\mathrm{Csp}^{2}-\mathrm{H}\right), 1604$ (C=C), 1486 ( $\mathrm{C}=\mathrm{C} \mathrm{Ar}$ ), 1456 ( $\mathrm{C}=\mathrm{C} \mathrm{Ar}), 1447$ ( $\mathrm{C}=\mathrm{C} \mathrm{Ar}) ;{ }^{1} \mathrm{H}$ NMR $\left(\mathrm{CDCl}_{3}, 400 \mathrm{MHz}\right) \delta_{\mathrm{H}} 8.22(\mathrm{br} \mathrm{s}, 1 \mathrm{H}, \mathrm{N}-$ H), $7.60-7.56(\mathrm{~m}, 2 \mathrm{H}, \mathrm{Ar}-\mathrm{H}), 7.48(\mathrm{dm}, J=8.1 \mathrm{~Hz}, 1 \mathrm{H}, \mathrm{Ar}-\mathrm{H}$ ), 7.43 (ddd, J=8.1, 0.9, $0.9 \mathrm{~Hz}, 1 \mathrm{H}$, Ar-H), $7.36-7.21$ (m, 7H, Ar-H), 7.12 (ddd, $J=8.1,7.1,0.9$ Hz, 1H, Ar-H), 7.03 (dd, $J=3.0,1.3$ $\mathrm{Hz}, 1 \mathrm{H}, \mathrm{Ar}-\mathrm{H}), 5.86(\mathrm{~d}, J=1.7 \mathrm{~Hz}, 1 \mathrm{H}, \mathrm{C}=\mathrm{CHH}), 5.32(\mathrm{~d}, J=1.7 \mathrm{~Hz}, 1 \mathrm{H}, \mathrm{C}=\mathrm{CHH}) ;{ }^{13} \mathrm{C} \mathrm{NMR}\left(\mathrm{CDCl}_{3}\right.$, $101 \mathrm{MHz}$ ) $\delta \mathrm{c} 142.7$ (C), 136.9 (C), 135.8 (C), 134.6 (C), 132.6 (C), 129.4 (C), 128.5 (CH), 127.6 $(\mathrm{CH}), 127.4(\mathrm{CH}), 126.2(\mathrm{CH}), 125.3(\mathrm{CH}), 123.0(\mathrm{CH}), 122.6(\mathrm{CH}), 120.2(\mathrm{CH}), 120.2(\mathrm{CH}), 115.6$ $\left(\mathrm{CH}_{2}\right), 114.9$ (C), $110.7(\mathrm{CH})$; HRMS (FTMS + c ESI) m/z: [M + H $]^{+}$Calcd for $\mathrm{C}_{20} \mathrm{H}_{16} \mathrm{NS} 302.0998$; Found 302.0996. 


\section{3,3'-(1-Phenylethane-1,1-diyl)bis(1H-indole) (3b) ${ }^{9}$}<smiles>CC(c1ccccc1)(c1ccccc1)c1c[nH]c2ccccc12</smiles>

An oven-dried vial was charged with the 2 -phenylindole ( $19.3 \mathrm{mg}, 0.100 \mathrm{mmol}, 1$ equiv.), phenylacetylene ( $30.6 \mathrm{mg}, 33 \mu \mathrm{L}, 0.3 \mathrm{mmol}, 3$ equiv.) and $\mathrm{PPh}_{3} \mathrm{AuNTf}_{2}$ (2:1 toluene adduct) (1.6 $\mathrm{mg}, 0.001 \mathrm{mmol}, 2 \mathrm{~mol} \%$ Au loading). The reagents were then dissolved in anhydrous MeCN $(0.3 \mathrm{~mL})$. The resulting solution was then stirred at $30{ }^{\circ} \mathrm{C}$ for $24 \mathrm{~h}$. Upon completion of the reaction the volatiles were removed under reduced pressure. To the crude residue was added indole ( $35.1 \mathrm{mg}, 0.3 \mathrm{mmol}, 3$ equiv.) and the mixture was then dissolved in MeCN (1.5 mL) before bistrifluoromethanesulfonimide $(4.2 \mathrm{mg}, 0.015 \mathrm{mmol}, 15 \mathrm{~mol} \%)$ was added and the resulting solution stirred at $30^{\circ} \mathrm{C}$ for $24 \mathrm{~h}$. The volatiles were then removed under reduced pressure. Purification of the resulting crude residue by silica gel column chromatography $(7: 1 \rightarrow 6: 1 \rightarrow 5: 1$ hexane/EtOAc) gave the product $\mathbf{3 b}(30.5 \mathrm{mg}, 0.091 \mathrm{mmol}, 91 \%)$ as an off white solid.

$\mathbf{R}_{\mathbf{f}} 0.20$ (5:1 hexane/EtOAc); $\mathbf{v}_{\max } / \mathrm{cm}^{-1} 3410(\mathrm{~N}-\mathrm{H}), 3051$ (Csp $\left.{ }^{2}-\mathrm{H}\right), 3013$ (Csp $\left.{ }^{2}-\mathrm{H}\right), 2978$ (Csp ${ }^{3}-$ $\mathrm{H}), 1538$ (C=C Ar), 1487 (C=C Ar), 1457 (C=C Ar), 1443 (C=C Ar); ${ }^{1} \mathbf{H}$ NMR (CDCl, $\left.300 \mathrm{MHz}\right) \delta_{\mathrm{H}}$ 7.80 (br s, 2H, N-H), $7.45-7.38$ (m, 2H, Ar-H), $7.36-7.32(\mathrm{~m}, 4 \mathrm{H}, \mathrm{Ar}-\mathrm{H}), 7.30-7.20(\mathrm{~m}, 3 \mathrm{H}$, Ar-H), 7.15 (ddd, $J=8.1,7.0,1.1 \mathrm{~Hz}, 2 \mathrm{H}, \mathrm{Ar}-\mathrm{H}$ ), 6.95 (ddd, $J=8.1,7.0,1.1 \mathrm{~Hz}, 2 \mathrm{H}, \mathrm{Ar}-\mathrm{H}$ ), 6.61 (d, $J=2.5 \mathrm{~Hz}, 2 \mathrm{H}, \mathrm{Ar}-\mathrm{H}), 2.38\left(\mathrm{~s}, 3 \mathrm{H}, \mathrm{CH}_{3}\right) ;{ }^{13} \mathrm{C} \mathrm{NMR}\left(\mathrm{CDCl}_{3}, 75 \mathrm{MHz}\right) \delta_{\mathrm{c}} 148.0(\mathrm{C}), 137.1$ (C), $128.0(\mathrm{CH}), 127.8(\mathrm{CH}), 126.4(\mathrm{C}), 125.8(\mathrm{CH}), 124.7(\mathrm{C}), 123.3(\mathrm{CH}), 122.1(\mathrm{CH}), 121.5(\mathrm{CH})$, $118.9(\mathrm{CH}), 111.1(\mathrm{CH}), 43.7(\mathrm{C}), 28.7\left(\mathrm{CH}_{3}\right) ;$ m.p. $=114-115^{\circ} \mathrm{C}$ (lit. $\left.{ }^{11} 117^{\circ} \mathrm{C}\right)$.

\section{3,3'-(1-Phenylethane-1,1-diyl)bis(7-methyl-1H-indole) (3d)}

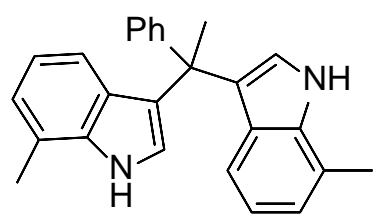

Following general procedure A, 2-methylindole ( $13.1 \mathrm{mg}, 0.100 \mathrm{mmol}, 1$ equiv.) was reacted with phenylacetylene $(30.6 \mathrm{mg}, 33 \mu \mathrm{L}, 0.3 \mathrm{mmol})$. After the reaction was complete, the volatiles were removed under reduced pressure. To the resulting residue was added 7- methylindole ( $26.2 \mathrm{mg}, 0.2 \mathrm{mmol}, 2$ equiv.) and the mixture was then dissolved in MeCN ( $2 \mathrm{~mL})$. The resulting solution was then allowed to equilibrate at $0{ }^{\circ} \mathrm{C}$ before $1,1^{\prime}$-binaphthyl-2,2'-diyl hydrogenphosphate $(3.5 \mathrm{mg}, 0.010 \mathrm{mmol}, 10 \mathrm{~mol} \%$ ) was added and the resulting solution stirred at $0{ }^{\circ} \mathrm{C}$ for $24 \mathrm{~h}$. After this time, the reaction was quenched by the addition of a few drops of triethylamine. The volatiles were then removed under reduced pressure. Purification of the resulting crude residue by silica gel column chromatography ( $4: 1$ toluene/hexane) gave the product 3d (39.7 $\mathrm{mg}, 0.082 \mathrm{mmol}, 82 \%$ ) as an off white solid. 
$\mathbf{R}_{\mathbf{f}} 0.21$ (4:1 toluene/hexane); $\boldsymbol{v}_{\max } / \mathrm{cm}^{-1} 3417(\mathrm{~N}-\mathrm{H}), 3135$ (Csp $\left.{ }^{2}-\mathrm{H}\right) 3049$ (Csp $\left.{ }^{2}-\mathrm{H}\right), 3027$ (Csp ${ }^{2}$ H) $2977\left(\mathrm{Csp}^{3}-\mathrm{H}\right), 2931\left(\mathrm{Csp}^{3}-\mathrm{H}\right), 2852\left(\mathrm{Csp}^{3}-\mathrm{H}\right), 1598$ (C=C Ar), 1586 (C=C Ar), 1492 (C=C Ar), 1455 (C=C Ar); ${ }^{1} \mathbf{H}$ NMR $\left(\mathrm{CDCl}_{3}, 400 \mathrm{MHz}\right) \delta_{\mathrm{H}} 7.60$ (br s, 2H, N-H), $7.42-7.36(\mathrm{~m}, 2 \mathrm{H}, \mathrm{Ar}-\mathrm{H})$, $7.26-7.18(\mathrm{~m}, 5 \mathrm{H}, \mathrm{Ar}-\mathrm{H}), 6.99-6.95(\mathrm{~m}, 2 \mathrm{H}, \mathrm{Ar}-\mathrm{H}), 6.91-6.86(\mathrm{~m}, 2 \mathrm{H}, \mathrm{Ar}-\mathrm{H}), 6.50$ (d, J = 2.5 $\mathrm{Hz}, 2 \mathrm{H}, \mathrm{Ar}-\mathrm{H}), 2.46\left(\mathrm{~s}, 6 \mathrm{H}, \mathrm{ArCH}_{3}\right), 2.36\left(\mathrm{~s}, 3 \mathrm{H}, \mathrm{CH}_{3}\right) ;{ }^{13} \mathrm{C} \mathrm{NMR}\left(\mathrm{CDCl}_{3}, 101 \mathrm{MHz}\right) \delta_{c} 148.1(\mathrm{C})$, 136.6 (C), $128.1(\mathrm{CH}), 127.7(\mathrm{CH}), 125.9(\mathrm{C}), 125.7(\mathrm{CH}), 125.2(\mathrm{C}), 123.2(\mathrm{CH}), 122.0(\mathrm{CH}), 120.2$ (C), $119.9(\mathrm{CH}), 119.0(\mathrm{CH}), 43.8(\mathrm{C}), 28.7\left(\mathrm{CH}_{3}\right), 16.6\left(\mathrm{CH}_{3}\right)$; m.p. = 196-198 ${ }^{\circ} \mathrm{C}$; HRMS (FTMS + p APCl corona) m/z: [M] ${ }^{+}$Calcd for $\mathrm{C}_{26} \mathrm{H}_{24} \mathrm{~N}_{2}$ 364.1934; Found 364.1935.

\section{3-(1-(1H-Indol-3-yl)-1-phenylethyl)-1-methyl-1H-indole (5a) ${ }^{9}$}

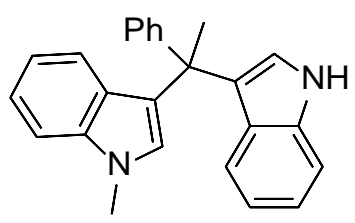

Following general procedure C, 2-(4,4,5,5-tetramethyl-1,3,2-dioxaborolan-2-yl)- $1 H$-indole ( $24.3 \mathrm{mg}, 0.100 \mathrm{mmol}$ ) was reacted with phenylacetylene (30.6 mg, $33 \mu \mathrm{L}, 0.3 \mathrm{mmol}$ ). After the reaction was complete, volatiles were removed under reduced pressure. The resulting residue was then dissolved in dichloromethane $(1 \mathrm{~mL})$ and silica gel was added until it formed a slurry. The resulting slurry was then stirred at room temperature for $4 \mathrm{~h}$. the slurry was then diluted with more dichloromethane and filtered, before the filter cake was washed several times with dichloromethane. The combined organics were then concentrated under reduced pressure to give a crude residue. To this crude residue was added 1- methylindole $(26.2 \mathrm{mg}$, $25 \mu \mathrm{L}, 0.2 \mathrm{mmol}, 2$ equiv.), and the mixture was then dissolved in $\mathrm{MeCN}(2 \mathrm{~mL})$. The resulting solution was then allowed to equilibrate at $0{ }^{\circ} \mathrm{C}$ before $1,1^{\prime}$-binaphthyl-2,2'-diyl hydrogenphosphate $(3.5 \mathrm{mg}, 0.010 \mathrm{mmol}, 10 \mathrm{~mol} \%)$ was added and the resulting solution stirred at $0{ }^{\circ} \mathrm{C}$ for $24 \mathrm{~h}$. After this time, the reaction was quenched by the addition of a few drops of triethylamine. The volatiles were then removed under reduced pressure. Purification of the resulting crude residue by silica gel column chromatography (1:1 toluene/hexane) gave the product 5 a (20.6 $\mathrm{mg}, 0.059 \mathrm{mmol}, 59 \%)$ as a yellow oil.

$\mathbf{R}_{\mathbf{f}} 0.14$ (1:1 toluene/hexane); $\mathbf{v}_{\max } / \mathrm{cm}^{-1} 3411(\mathrm{~N}-\mathrm{H}), 3087$ (Csp $\left.{ }^{2}-\mathrm{H}\right), 3053\left(\mathrm{Csp}^{2}-\mathrm{H}\right), 3027$ (Csp ${ }^{2}-$ H), 2977 ( $\mathrm{Csp}^{3}-\mathrm{H}$ ), 2936 ( $\mathrm{Csp}^{3}-\mathrm{H}$ ), 1540 (C=C Ar), 1483 (C=C Ar), 1456 (C=C Ar), 1443 (C=C Ar); ${ }^{1} \mathrm{H}$ NMR $\left(\mathrm{CDCl}_{3}, 400 \mathrm{MHz}\right) \delta_{\mathrm{H}} 7.82(\mathrm{br} \mathrm{s}, 1 \mathrm{H}, \mathrm{N}-\mathrm{H}), 7.43-7.39(\mathrm{~m}, 2 \mathrm{H}, \mathrm{Ar}-\mathrm{H}), 7.36-7.26(\mathrm{~m}$, $5 \mathrm{H}, \mathrm{Ar}-\mathrm{H}$ ), $7.25-7.13(\mathrm{~m}, 4 \mathrm{H}, \mathrm{Ar}-\mathrm{H}), 6.94(\mathrm{ddt}, J=8.2,7.0,1.2 \mathrm{~Hz}, 2 \mathrm{H}, \mathrm{Ar}-\mathrm{H}), 6.65$ (d, J = 2.5 $\mathrm{Hz}, 1 \mathrm{H}, \mathrm{Ar}-\mathrm{H}), 6.47$ (s, $1 \mathrm{H}, \mathrm{Ar}-\mathrm{H}), 3.67\left(\mathrm{~s}, 3 \mathrm{H}, \mathrm{NCH}_{3}\right), 2.38$ (s, 3H, $\left.\mathrm{ArCH}_{3}\right) . ;{ }^{13} \mathrm{C} \mathrm{NMR}\left(\mathrm{CDCl}_{3}, 101\right.$ $\mathrm{MHz}$ ) $\delta_{\mathrm{c}} 148.2(\mathrm{C}), 137.8(\mathrm{C}), 137.2(\mathrm{C}), 128.1(\mathrm{CH}), 128.0(\mathrm{CH}), 127.7(\mathrm{CH}), 126.9(\mathrm{C}), 126.5(\mathrm{C})$, $125.7(\mathrm{CH}), 124.9(\mathrm{C}), 123.4(\mathrm{CH}), 123.4(\mathrm{C}), 122.2(\mathrm{CH}), 122.1(\mathrm{CH}), 121.5(\mathrm{CH}), 121.1(\mathrm{CH})$, $118.9(\mathrm{CH}), 118.4(\mathrm{CH}), 111.1(\mathrm{CH}), 109.1(\mathrm{CH}), 43.8(\mathrm{C}), 32.6\left(\mathrm{CH}_{3}\right), 29.0\left(\mathrm{CH}_{3}\right)$.

\section{3-(1-(1H-Indol-3-yl)-1-phenylethyl)-7-methyl-1H-indole (5b) ${ }^{12}$}




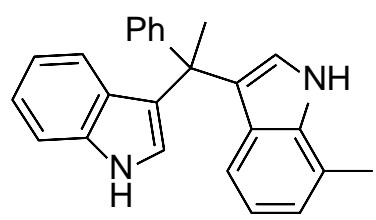

Following general procedure C, 2-(4,4,5,5-tetramethyl-1,3,2-dioxaborolan-2-yl)-1H-indole ( $24.3 \mathrm{mg}, 0.100 \mathrm{mmol}$ ) was reacted with phenylacetylene $(30.6 \mathrm{mg}, 33 \mu \mathrm{L}, 0.3 \mathrm{mmol}$ ). After the reaction was complete the volatiles were removed under reduced pressure. The resulting residue was then dissolved in dichloromethane $(1 \mathrm{~mL})$ and silica gel was added until it formed a slurry. The resulting slurry was then stirred at room temperature for $4 \mathrm{~h}$. the slurry was then diluted with more dichloromethane and filtered, before the filter cake was washed several times with dichloromethane. The combined organics were then concentrated at under reduced pressure to give a crude residue. To this crude residue was added 7-methylindole (26.2 $\mathrm{mg}, 0.2 \mathrm{mmol}, 2$ equiv.), the mixture was then dissolved in MeCN $(2 \mathrm{~mL})$. The resulting solution was then allowed to equilibrate at $0{ }^{\circ} \mathrm{C}$ before 1,1'-binaphthyl-2,2'-diyl hydrogenphosphate $\left(3.5 \mathrm{mg}, 0.010 \mathrm{mmol}, 10 \mathrm{~mol} \%\right.$ ) was added and the resulting solution stirred at $0{ }^{\circ} \mathrm{C}$ for $24 \mathrm{~h}$. After this time, the reaction was quenched by the addition of a few drops of triethylamine. The volatiles were then removed under reduced pressure. Purification of the resulting crude residue by silica gel column chromatography ( $4: 1$ toluene/hexane) gave the product $\mathbf{5 b}$ (19.0 mg, $0.054 \mathrm{mmol}, 54 \%)$ as a yellow solid.

$\mathbf{R}_{\mathbf{f}} 0.19$ (4:1 toluene/hexane); $\boldsymbol{v}_{\max } / \mathrm{cm}^{-1} 3410(\mathrm{~N}-\mathrm{H}), 3052$ (Csp $\left.{ }^{2}-\mathrm{H}\right), 3016$ (Csp $\left.{ }^{2}-\mathrm{H}\right), 2974$ (Csp 3 H), 2932 ( $\mathrm{Csp}^{3}-\mathrm{H}$ ), 1597 (C=C Ar), 1538 ( $\mathrm{C}=\mathrm{C} \mathrm{Ar}$ ), 1491 (C=C Ar), 1455 ( $\mathrm{C}=\mathrm{C} \mathrm{Ar}$ ), 1443 ( $\mathrm{C}=\mathrm{C} \mathrm{Ar}$ ); ${ }^{1} \mathrm{H}$ NMR $\left(\mathrm{CDCl}_{3}, 400 \mathrm{MHz}\right) \delta_{\mathrm{H}} 7.80(\mathrm{br} \mathrm{s}, 1 \mathrm{H}, \mathrm{N}-\mathrm{H}), 7.72(\mathrm{br} \mathrm{s}, 1 \mathrm{H}, \mathrm{N}-\mathrm{H}), 7.43-7.38(\mathrm{~m}, 2 \mathrm{H}, \mathrm{Ar}-$ H), $7.37-7.32(\mathrm{~m}, 2 \mathrm{H}, \mathrm{Ar}-\mathrm{H}), 7.26-7.12(\mathrm{~m}, 5 \mathrm{H}, \mathrm{Ar}-\mathrm{H}), 6.97-6.92(\mathrm{~m}, 2 \mathrm{H}, \mathrm{Ar}-\mathrm{H}), 6.90-6.85$ $(\mathrm{m}, 1 \mathrm{H}, \mathrm{Ar}-\mathrm{H}), 6.62-6.57(\mathrm{~m}, 2 \mathrm{H}, \mathrm{Ar}-\mathrm{H}), 2.47\left(\mathrm{~s}, 3 \mathrm{H}, \mathrm{CH}_{3}\right), 2.37\left(\mathrm{~s}, 3 \mathrm{H}, \mathrm{CH}_{3}\right) ;{ }^{13} \mathrm{C} \mathrm{NMR}\left(\mathrm{CDCl}_{3}\right.$,

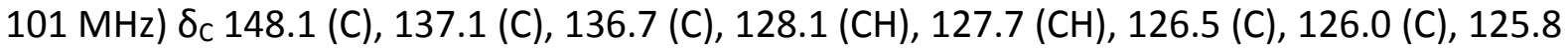
$(\mathrm{CH}), 125.3(\mathrm{C}), 124.9(\mathrm{C}), 123.4(\mathrm{CH}), 123.1(\mathrm{CH}), 122.1(\mathrm{CH}), 122.1(\mathrm{CH}), 121.5(\mathrm{CH}), 120.1(\mathrm{C})$, $119.9(\mathrm{CH}), 119.1(\mathrm{CH}), 118.9(\mathrm{CH}), 111.1(\mathrm{CH}), 43.8(\mathrm{C}), 28.8\left(\mathrm{CH}_{3}\right), 16.6\left(\mathrm{CH}_{3}\right) ;$ m.p. $=123-125$ ${ }^{\circ} \mathrm{C}$ (lit. $\left.{ }^{13} 124-125^{\circ} \mathrm{C}\right)$.

\section{3-(1-(1H-Indol-3-yl)-1-phenylethyl)-6-fluoro-1H-indole $(5 c)^{13}$}

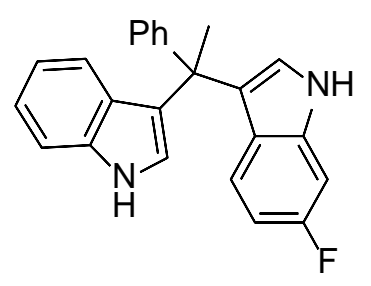

Following general procedure C, 2-(4,4,5,5-tetramethyl-1,3,2-dioxaborolan-2-yl)-1H-indole ( $24.3 \mathrm{mg}, 0.100 \mathrm{mmol}$ ) was reacted with phenylacetylene $(30.6 \mathrm{mg}, 33 \mu \mathrm{L}, 0.3 \mathrm{mmol}$ ). After the reaction was complete the volatiles were removed under reduced pressure. The resulting residue was then dissolved in dichloromethane $(1 \mathrm{~mL})$ and silica gel was added until it formed a slurry. The resulting slurry was then stirred at room temperature for $4 \mathrm{~h}$. the slurry was then diluted with more dichloromethane and filtered, before the filter cake was washed several 
times with dichloromethane. The combined organics were then concentrated at under reduced pressure to give a crude residue. To this crude residue was added 6-fluoroindole (27.0 $\mathrm{mg}, 0.2 \mathrm{mmol}, 2$ equiv.), and the mixture was then dissolved in MeCN ( $2 \mathrm{~mL})$. The resulting solution was then allowed to equilibrate at $0{ }^{\circ} \mathrm{C}$ before $1,1^{\prime}$-binaphthyl-2,2'-diyl hydrogenphosphate $(3.5 \mathrm{mg}, 0.010 \mathrm{mmol}, 10 \mathrm{~mol} \%)$ was added and the resulting solution stirred at $0{ }^{\circ} \mathrm{C}$ for $24 \mathrm{~h}$. After this time, the reaction was quenched by the addition of a few drops of triethylamine. The volatiles were then removed under reduced pressure. Purification of the resulting crude residue by silica gel column chromatography ( $7: 3$ toluene/hexane) gave the product $5 \mathrm{c}(21.1 \mathrm{mg}, 0.060 \mathrm{mmol}, 60 \%)$ as a yellow solid.

$\mathbf{R}_{\mathbf{f}} 0.16$ (7:3 toluene/hexane); $\mathbf{v}_{\max } / \mathrm{cm}^{-1} 3415(\mathrm{~N}-\mathrm{H}), 3056$ (Csp $\left.{ }^{2}-\mathrm{H}\right), 3013\left(\mathrm{Csp}^{2}-\mathrm{H}\right), 2979$ (Csp ${ }^{3}-$ H), 2936 ( $\mathrm{Csp}^{3}-\mathrm{H}$ ), 1543 (C=C Ar), 1494 (C=C Ar), 1456 (C=C Ar); ${ }^{1} \mathbf{H}$ NMR (CDCl, $\left.400 \mathrm{MHz}\right) \delta_{\mathrm{H}}$ 7.86 (br s, 1H, N-H), 7.81 (br s, 1H, N-H), $7.42-7.30(\mathrm{~m}, 5 \mathrm{H}, \mathrm{Ar}-\mathrm{H}), 7.23-7.12(\mathrm{~m}, 4 \mathrm{H}, \mathrm{Ar}-\mathrm{H})$, $7.02-6.98(\mathrm{~m}, 1 \mathrm{H}, \mathrm{Ar}-\mathrm{H}), 6.97-6.92(\mathrm{~m}, 1 \mathrm{H}, \mathrm{Ar}-\mathrm{H}), 6.72-6.66(\mathrm{~m}, 1 \mathrm{H}, \operatorname{Ar}-\mathrm{H}), 6.64(\mathrm{~d}, J=2.5$ $\mathrm{Hz}, 1 \mathrm{H}, \mathrm{Ar}-\mathrm{H}), 6.62$ (d, $J=2.5 \mathrm{~Hz}, 1 \mathrm{H}, \mathrm{Ar}-\mathrm{H}), 2.35\left(\mathrm{~s}, 3 \mathrm{H}, \mathrm{CH}_{3}\right) ;{ }^{13} \mathrm{C} \mathrm{NMR}\left(\mathrm{CDCl}_{3}, 101 \mathrm{MHz}\right) \delta_{\mathrm{C}}$ $159.6\left(\mathrm{~d}, J_{C F}=237.8 \mathrm{~Hz}, \mathrm{C}\right), 147.8(\mathrm{C}), 137.1(\mathrm{C}), 137.0$ (d, $\left.J_{C F}=12.9 \mathrm{~Hz}, \mathrm{C}\right), 128.0(\mathrm{CH}), 127.8$ (CH), 126.4 (C), 125.9 (CH), 124.9 (C), 124.6 (C), 123.5 (d, J J $=3.5 \mathrm{~Hz}, \mathrm{CH}), 123.2(\mathrm{CH}), 123.1$ (C), $122.7\left(\mathrm{~d}, J_{C F}=9.8 \mathrm{~Hz}, \mathrm{CH}\right), 122.0(\mathrm{CH}), 121.6(\mathrm{CH}), 119.0(\mathrm{CH}), 111.1(\mathrm{CH}), 107.7\left(\mathrm{~d}, J_{C F}=\right.$ $24.3 \mathrm{~Hz}, \mathrm{CH}), 97.2\left(\mathrm{~d}, J_{C F}=25.7 \mathrm{~Hz}, \mathrm{CH}\right), 43.7(\mathrm{C}), 28.8\left(\mathrm{CH}_{3}\right) ;{ }^{19} \mathrm{~F} \mathrm{NMR}\left(\mathrm{CDCl}_{3}, 376 \mathrm{MHz}\right) \delta_{F}-$ 121.87 (ddd, $J_{H F}=9.3,9.3,5.3 \mathrm{~Hz}$ ); m.p. $=115-117^{\circ} \mathrm{C}$ (lit. $.^{13} 104-105^{\circ} \mathrm{C}$ ).

\section{3-(1-(1H-Indol-3-yl)-1-phenylethyl)-5-methoxy-1H-indole (5d) $)^{12}$}

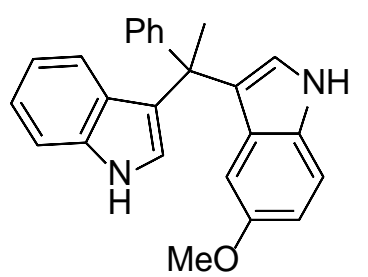

Following general procedure C, 2-(4,4,5,5-tetramethyl-1,3,2-dioxaborolan-2-yl)- $1 H$-indole ( $24.3 \mathrm{mg}, 0.100 \mathrm{mmol}$ ) was reacted with phenylacetylene (30.6 mg, $33 \mu \mathrm{L}, 0.3 \mathrm{mmol}$ ). After the reaction was complete the volatiles were removed under reduced pressure. The resulting residue was then dissolved in dichloromethane $(1 \mathrm{~mL})$ and silica gel was added until it formed a slurry. The resulting slurry was then stirred at room temperature for $4 \mathrm{~h}$. the slurry was then diluted with more dichloromethane and filtered, before the filter cake was washed several times with dichloromethane. The combined organics were then concentrated at under reduced pressure to give a crude residue. To this crude residue was added 5-methoxyindole (29.4 $\mathrm{mg}, 0.2 \mathrm{mmol}, 2$ equiv.), and the mixture was then dissolved in MeCN ( $2 \mathrm{~mL}$ ). The resulting solution was then allowed to equilibrate at $0{ }^{\circ} \mathrm{C}$ before $1,1^{\prime}$-binaphthyl-2,2'-diyl hydrogenphosphate $(3.5 \mathrm{mg}, 0.010 \mathrm{mmol}, 10 \mathrm{~mol} \%)$ was added and the resulting solution stirred at $0{ }^{\circ} \mathrm{C}$ for $24 \mathrm{~h}$. After this time, the reaction was quenched by the addition of a few drops of triethylamine. The volatiles were then removed under reduced pressure. Purification of the resulting crude residue by silica gel column chromatography (100:0 $\rightarrow 19: 1$ toluene/EtOAc) gave the product $\mathbf{5 d}(18.2 \mathrm{mg}, 0.050 \mathrm{mmol}, 50 \%)$ as a yellow oil. 
$\mathbf{R}_{\mathbf{f}} 0.21$ (toluene); $\mathbf{v}_{\max } / \mathrm{cm}^{-1} 3412(\mathrm{~N}-\mathrm{H}), 3054\left(\mathrm{Csp}^{2}-\mathrm{H}\right), 3029$ (Csp $\left.{ }^{2}-\mathrm{H}\right), 2933\left(\mathrm{Csp}^{3}-\mathrm{H}\right), 2873$ ( $\mathrm{Csp}^{3}-\mathrm{H}$ ), 1598 ( $\mathrm{C}=\mathrm{C} \mathrm{Ar}$ ), 1579 ( $\mathrm{C}=\mathrm{C} \mathrm{Ar}$ ), 1481 (C=C Ar), 1455 (C=C Ar), 1441 (C=C Ar), 1248 (CO-C), 1100 (C-O-C); ${ }^{1} \mathrm{H}$ NMR $\left(\mathrm{CDCl}_{3}, 300 \mathrm{MHz}\right.$ ) $\delta_{\mathrm{H}} 7.87$ (br s, $\left.1 \mathrm{H}, \mathrm{N}-\mathrm{H}\right), 7.78$ (br s, $\left.1 \mathrm{H}, \mathrm{N}-\mathrm{H}\right), 7.44$ - $7.31(\mathrm{~m}, 4 \mathrm{H}, \mathrm{Ar}-\mathrm{H}), 7.27-7.11(\mathrm{~m}, 5 \mathrm{H}, \mathrm{Ar}-\mathrm{H}), 6.94$ (ddd, J = 8.3, 7.1, 1.0 Hz, 1H, Ar-H), 6.79 (dd, J = 8.8, 2.5 Hz, 1H, Ar-H), 6.70 (d, J = 2.5 Hz, 1H, Ar-H), 6.67 (d, J = 2.5 Hz, 1H, Ar-H), 6.65 (d, $J=2.5 \mathrm{~Hz}, 1 \mathrm{H}, \mathrm{Ar}-\mathrm{H}), 3.60\left(\mathrm{~s}, 3 \mathrm{H}, \mathrm{OCH}_{3}\right), 2.36\left(\mathrm{~s}, 3 \mathrm{H}, \mathrm{CH}_{3}\right) ;{ }^{13} \mathrm{C} \mathrm{NMR}\left(\mathrm{CDCl}_{3}, 75 \mathrm{MHz}\right) \delta \mathrm{c} 153.2$ (C), 147.9 (C), 137.1 (C), 132.2 (C), 128.1 (CH), 127.7 (CH), 126.9 (C), 126.5 (C), 125.8 (CH), $124.6(\mathrm{C}), 124.5(\mathrm{C}), 123.9(\mathrm{CH}), 123.4(\mathrm{CH}), 122.1(\mathrm{CH}), 121.5(\mathrm{CH}), 118.9(\mathrm{CH}), 111.6(\mathrm{CH})$, $111.5(\mathrm{CH}), 111.1(\mathrm{CH}), 104.1(\mathrm{CH}), 55.7\left(\mathrm{CH}_{3}\right), 43.7(\mathrm{C}), 28.7\left(\mathrm{CH}_{3}\right)$.

\section{6-Fluoro-3-(1-(1-methyl-1H-indol-3-yl)-1-phenylethyl)-1H-indole (5e)}

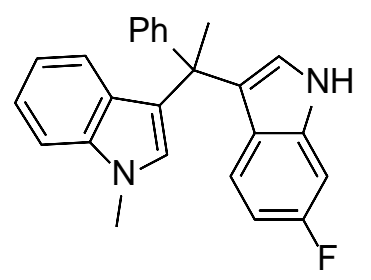

Following general procedure C, 1-methyl-2-(4,4,5,5-tetramethyl-1,3,2-dioxaborolan-2-yl)-1Hindole $(25.7 \mathrm{mg}, 0.100 \mathrm{mmol})$ was reacted with phenylacetylene $(30.6 \mathrm{mg}, 33 \mu \mathrm{L}, 0.3 \mathrm{mmol})$. After the reaction was complete the volatiles were removed under reduced pressure. The resulting residue was then dissolved in dichloromethane $(1 \mathrm{~mL})$ and silica gel was added until it formed a slurry. The resulting slurry was then stirred at room temperature for $4 \mathrm{~h}$. the slurry was then diluted with more dichloromethane and filtered, before the filter cake was washed several times with dichloromethane. The combined organics were then concentrated at under reduced pressure to give a crude residue. To this crude residue was added 6-fluoroindole (27.0 $\mathrm{mg}, 0.2 \mathrm{mmol}, 2$ equiv.), and the mixture was then dissolved in $\mathrm{MeCN}(2 \mathrm{~mL})$. The resulting solution was then allowed to equilibrate at $0{ }^{\circ} \mathrm{C}$ before $1,1^{\prime}$-binaphthyl-2,2'-diyl hydrogenphosphate $(3.5 \mathrm{mg}, 0.010 \mathrm{mmol}, 10 \mathrm{~mol} \%)$ was added and the resulting solution stirred at $0{ }^{\circ} \mathrm{C}$ for $24 \mathrm{~h}$. After this time, the reaction was quenched by the addition of a few drops of triethylamine. The volatiles were then removed under reduced pressure. Purification of the resulting crude residue by silica gel column chromatography (1:1 hexane/toluene) gave the product 5 e $(24.9 \mathrm{mg}, 0.068 \mathrm{mmol}, 68 \%)$ as an off-white solid.

$\mathbf{R}_{\mathbf{f}} 0.20$ (1:1 hexane/toluene); $\mathbf{v}_{\max } / \mathrm{cm}^{-1} 3409(\mathrm{~N}-\mathrm{H}), 3053$ (Csp $\left.{ }^{2}-\mathrm{H}\right), 3024\left(\mathrm{Csp}^{2}-\mathrm{H}\right), 2974$ (Csp ${ }^{3}-$ H), 2930 ( $\mathrm{Csp}^{3}-\mathrm{H}$ ), 1599 (C=C Ar), 1541 (C=C Ar), 1494 (C=C Ar), 1484 (C=C Ar), 1456 (C=C Ar); ${ }^{1} \mathbf{H}$ NMR $\left(\mathrm{CDCl}_{3}, 300 \mathrm{MHz}\right) \delta_{\mathrm{H}} 7.84(\mathrm{br} \mathrm{s}, 1 \mathrm{H}, \mathrm{N}-\mathrm{H}), 7.41-7.27(\mathrm{~m}, 5 \mathrm{H}, \mathrm{Ar}-\mathrm{H}), 7.25-7.13$ (m, $4 \mathrm{H}, \mathrm{Ar}-\mathrm{H}$ ), 7.01 (ddd, $J=9.6,2.4,0.5 \mathrm{~Hz}, 1 \mathrm{H}, \mathrm{Ar}-\mathrm{H}$ ), 6.93 (ddd, $J=8.1,6.9,1.1 \mathrm{~Hz}, 1 \mathrm{H}, \mathrm{Ar}-\mathrm{H}$ ), $6.72-6.64(\mathrm{~m}, 2 \mathrm{H}, \mathrm{Ar}-\mathrm{H}), 6.44(\mathrm{~s}, 1 \mathrm{H}, \mathrm{Ar}-\mathrm{H}), 3.68\left(\mathrm{~s}, 3 \mathrm{H}, \mathrm{N}-\mathrm{CH}_{3}\right), 2.34\left(\mathrm{~s}, 3 \mathrm{H}, \mathrm{CH}_{3}\right) ;{ }^{13} \mathrm{C} \mathrm{NMR}$ $\left(\mathrm{CDCl}_{3}, 75 \mathrm{MHz}\right) \delta_{\mathrm{C}} 159.5$ (d, $\left.J_{C F}=237.6 \mathrm{~Hz}, \mathrm{C}\right), 147.9$ (C), 137.7 (C), 137.0 (d, $\left.J_{C F}=12.2 \mathrm{~Hz}, \mathrm{C}\right)$, $128.0(\mathrm{CH}), 128.0(\mathrm{CH}), 127.8(\mathrm{CH}), 126.8(\mathrm{C}), 125.8(\mathrm{CH}), 124.9(\mathrm{C}), 123.5\left(\mathrm{~d}, J_{C F}=3.4 \mathrm{~Hz}, \mathrm{CH}\right)$, $123.1(\mathrm{C}), 123.0(\mathrm{C}), 122.6\left(\mathrm{~d}, J_{C F}=9.9 \mathrm{~Hz}, \mathrm{CH}\right), 122.1(\mathrm{CH}), 121.1(\mathrm{CH}), 118.4(\mathrm{CH}), 109.2(\mathrm{CH})$, $107.7\left(\mathrm{~d}, J_{C F}=23.9 \mathrm{~Hz}, \mathrm{CH}\right), 97.2\left(\mathrm{~d}, J_{C F}=25.9 \mathrm{~Hz}, \mathrm{CH}\right), 43.6(\mathrm{C}), 32.6\left(\mathrm{CH}_{3}\right), 29.0\left(\mathrm{CH}_{3}\right) ;{ }^{19} \mathrm{~F} \mathrm{NMR}$ 
$\left(\mathrm{CDCl}_{3}, 282 \mathrm{MHz}\right) \delta_{\mathrm{F}}-121.95\left(\mathrm{ddd}, J_{H F}=9.5,9.5,5.5 \mathrm{~Hz}\right) ;$ m.p. $=89-91^{\circ} \mathrm{C} ; \mathrm{HRMS}$ (FTMS + p APCl corona) m/z: [M] ${ }^{+}$. Calcd for $\mathrm{C}_{25} \mathrm{H}_{21} \mathrm{~N}_{2} \mathrm{~F}$ 368.1683; Found 368.1685. 
NMR Spectra

ebmh223char.1.fid

$1 \mathrm{H} 400.1 \mathrm{MHz}$ J ob $36817 \mathrm{McLean}$ Euan B $223 \mathrm{CHAR} \mathrm{CDCl} 325.0^{\circ} \mathrm{C}$

proton characterisation

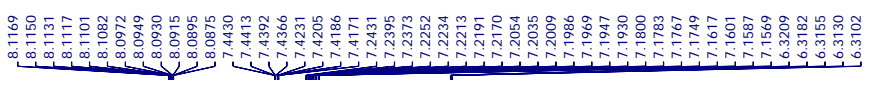

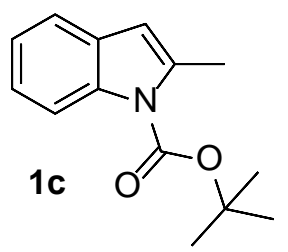

ebmc223char.1.fid

13C 100.6MHz J ob 36829 McLean Euan B 223CHAR CDCl3 $25.0^{\circ} \mathrm{C} \quad 0$ hour $29 \mathrm{~min}$

carbon characterisation

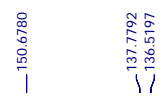

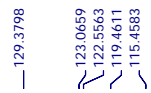

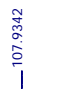

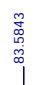

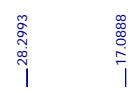
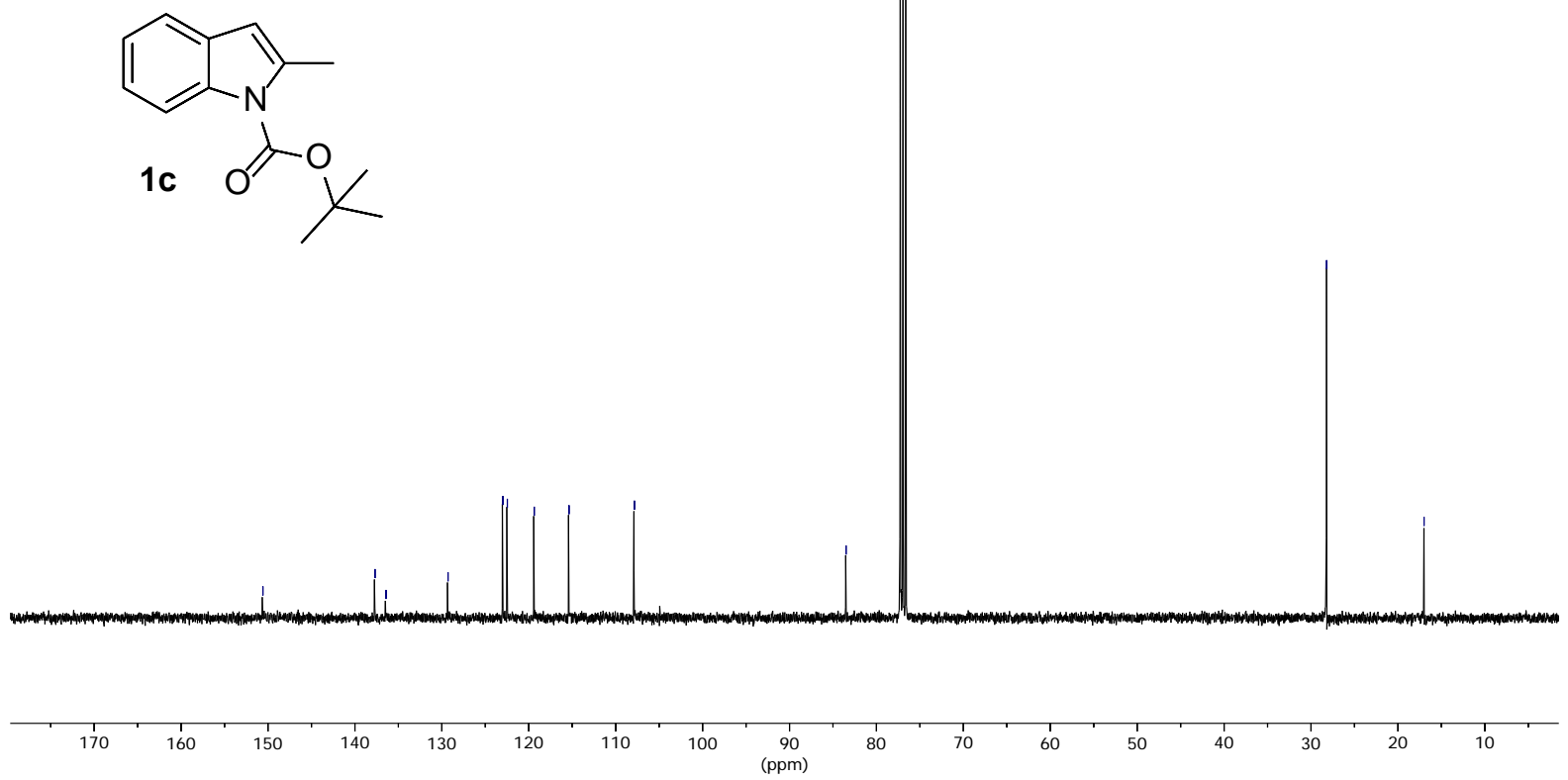

S-29 
ebmh270char1.1.fid

1H 400.1MHz Job 37761 McLean Euan B 270CHAR1 CDCl3 $25.0 \bigcirc$

proton characterisation

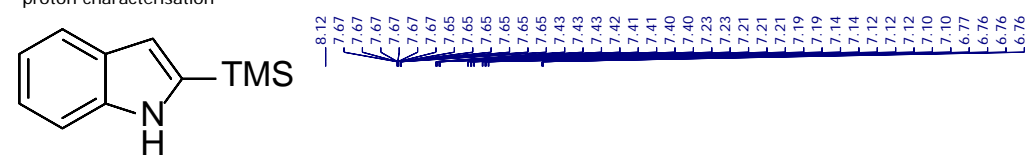

11

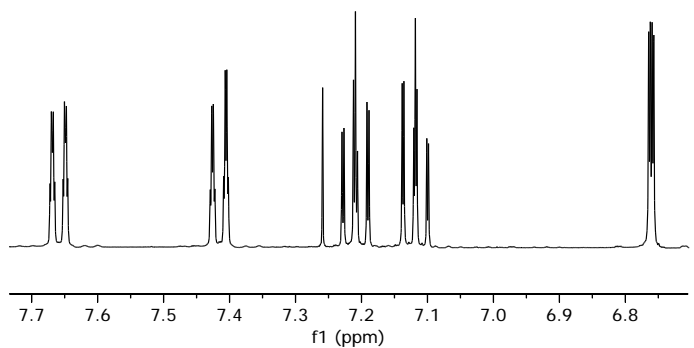

\begin{tabular}{cccccccccccccccc}
\hline & & & \\
\hline
\end{tabular}

ebmc270char.1.fid

13C 75.5MHz Job 85260 McLean Euan B 270CHAR CDCl3 $22.7 \diamond \mathrm{C} \quad 1$ hour $30 \mathrm{~min}$ carbon characterisation<smiles>[As]c1cc2ccccc2[nH]1</smiles>

11

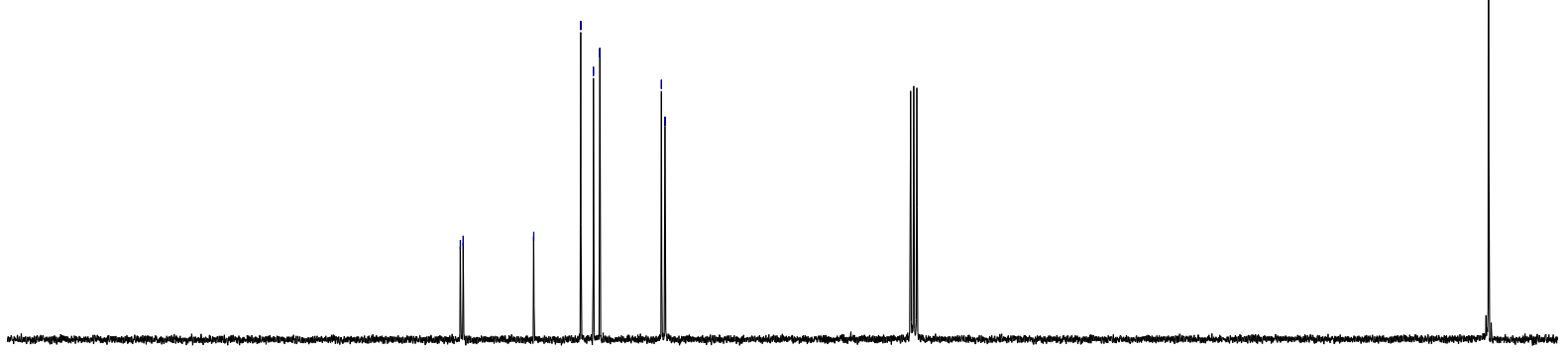

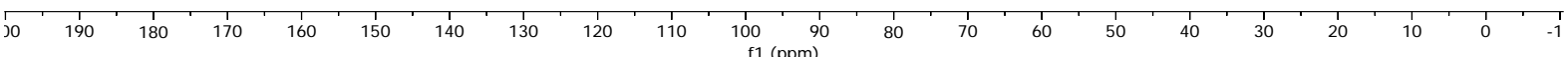


f5chfmc34c.1.fid

1H 300.1MHz Job 83145 Cutolo Francesca M FMC34C CDCl3 24.9

Pure compound

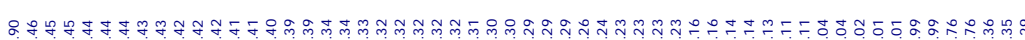<smiles>C=C(c1ccccc1)c1c(C)[nH]c2ccccc12</smiles>

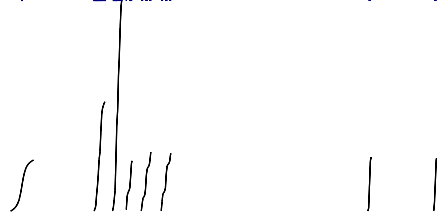

$4 a$

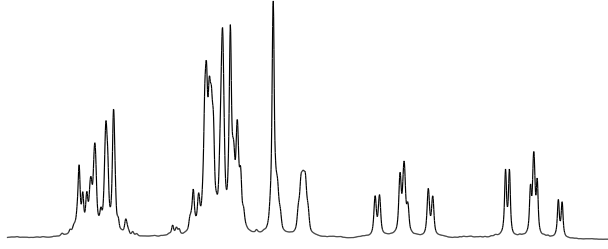

$\begin{array}{llllllllllllll}7.50 & 7.45 & 7.40 & 7.35 & 7.30 & 7.25 & 7.20 & 7.15 & 7.10 & 7.05 & 7.00 & 6.95\end{array}$

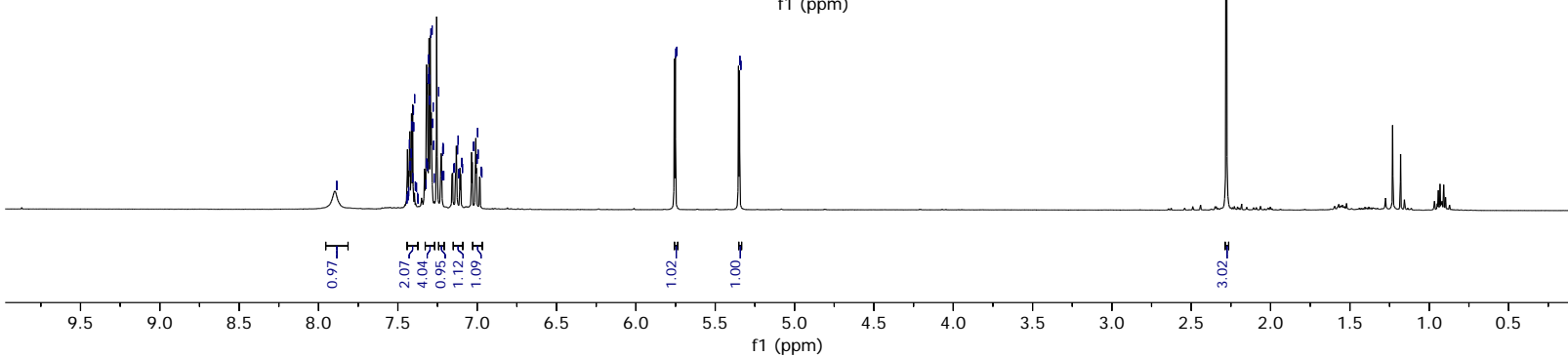

f5ccfmc34c.1. fid

13C 75.5MHz Job 83148 Cutolo Francesca M FMC34C CDCl3 25.1 2 hours 25 min FMC $34 \mathrm{C}$ pure compound

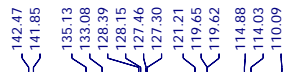<smiles>C=C(c1ccccc1)c1c(C)[nH]c2ccccc12</smiles>

$4 a$

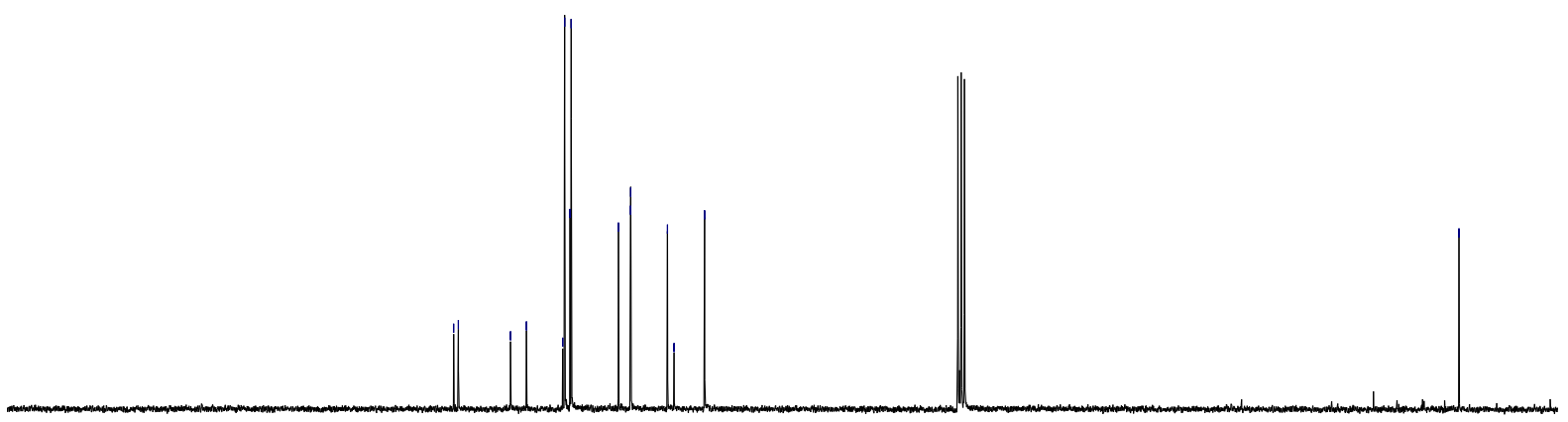

30

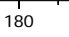

160

50

130

110 100
$\mathrm{f} 1$ (ppm) 
ebmh211bchar.1.fid

1H 400.1MHz J ob 36815 McLean Euan B 211BCHAR CDCl3 $25.0^{\circ} \mathrm{C}$

proton characterisation<smiles>C=C(c1ccccc1)c1c(C)n(C)c2ccccc12</smiles>

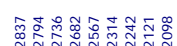<smiles></smiles>

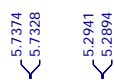

4b
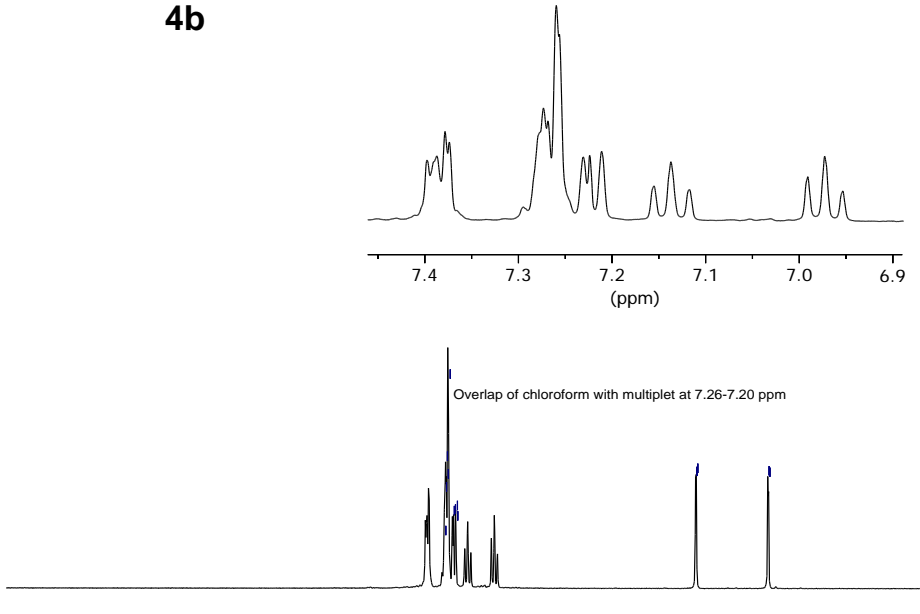

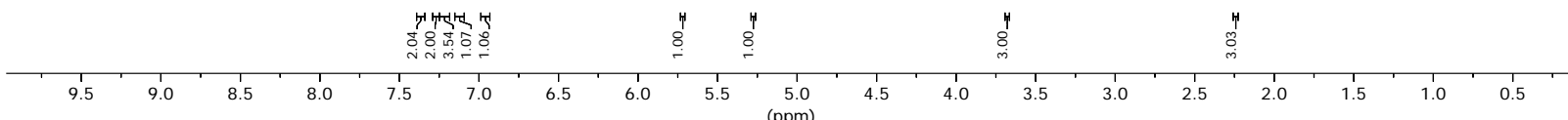

ebmc211bchar.1.fid

13C 100.6MHz J ob 36827 McLean Euan B 211BCHAR CDCl3 $25.0^{\circ} \mathrm{C} \quad 1$ hour $12 \mathrm{~min}$ carbon characterisation
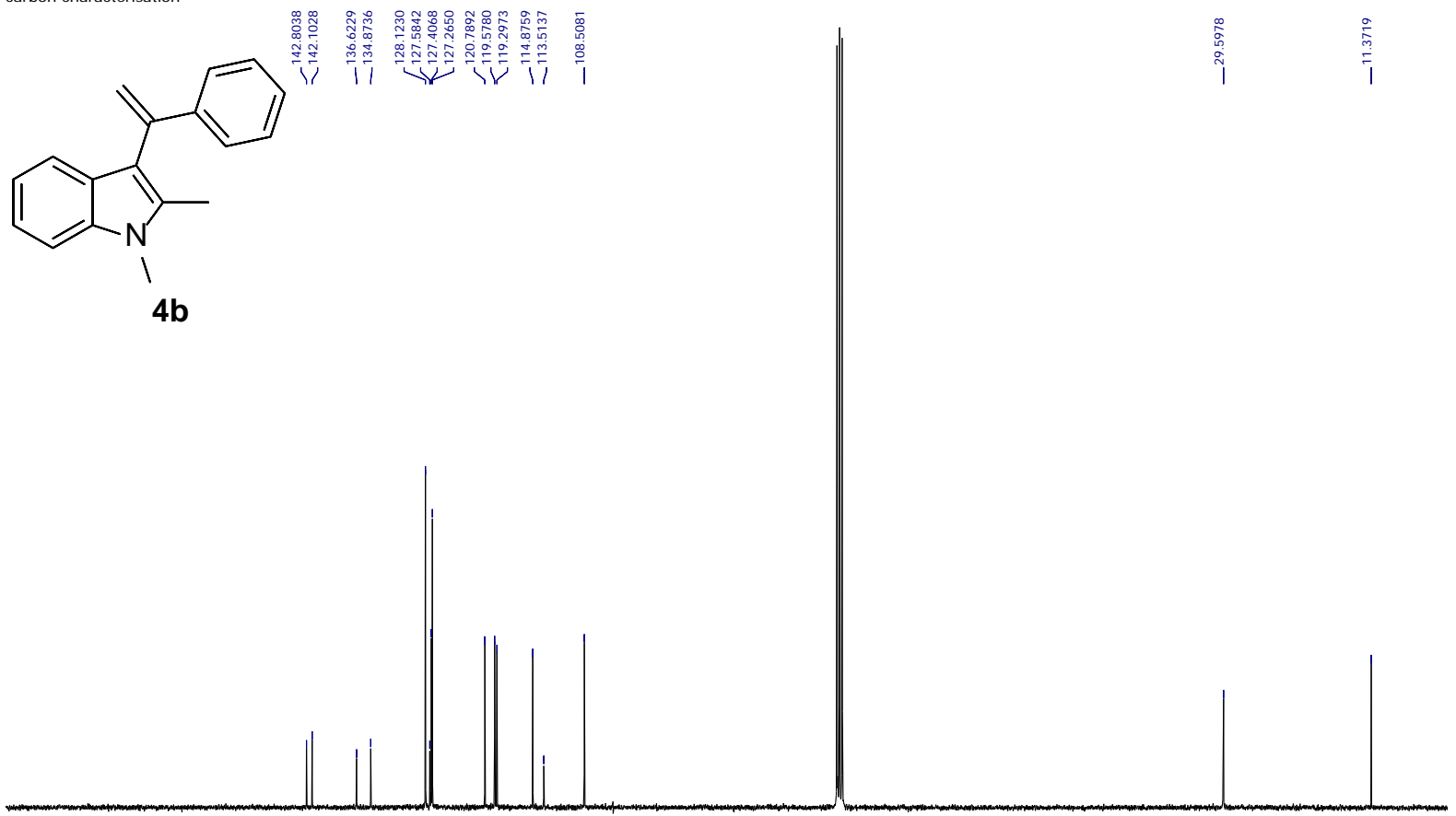

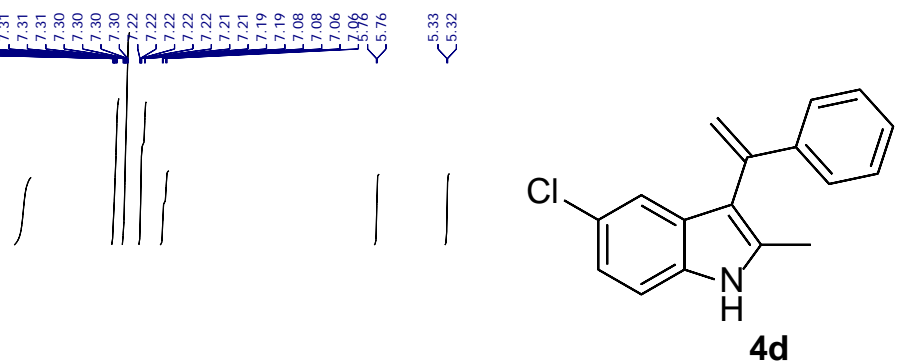

4d

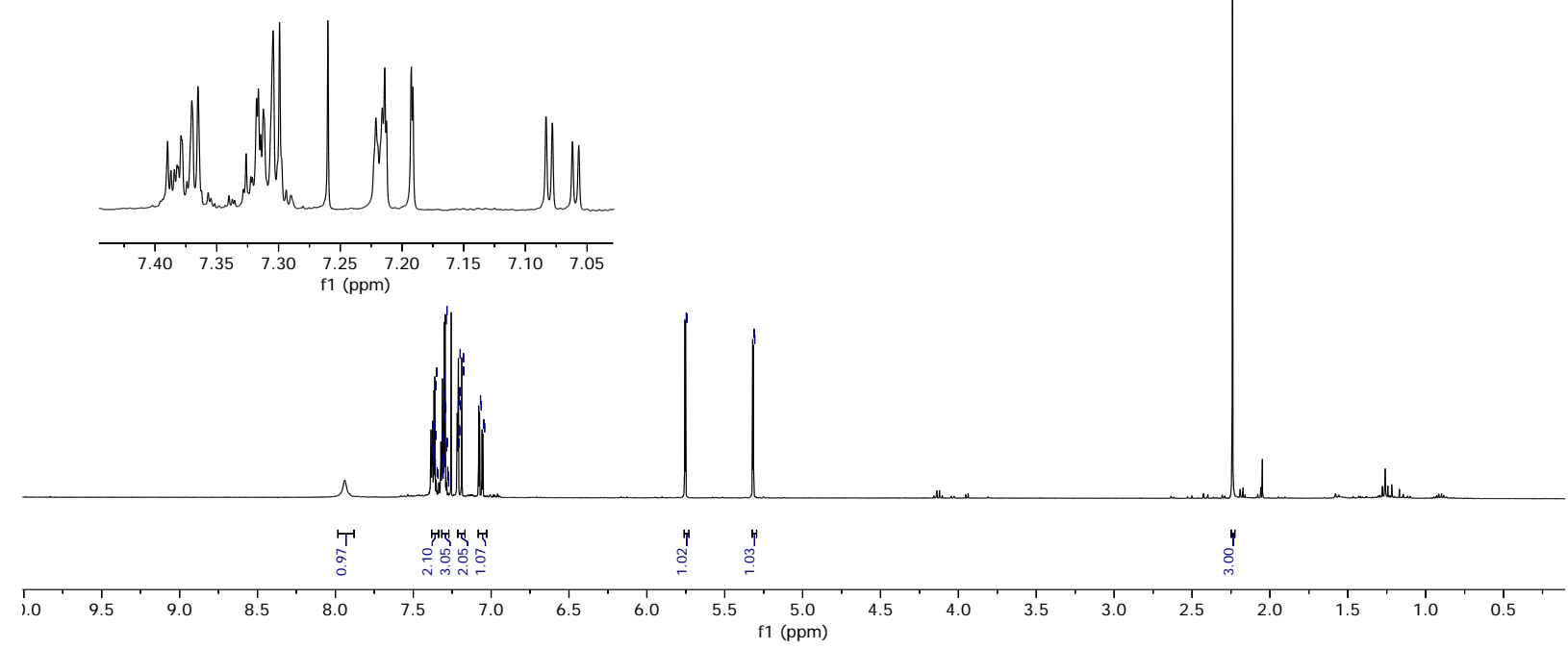

ebmc221char.1.fid

(3) 100.6MHZ J ob 36828 McLean Euan B 221CHAR CDCl3 $25.0^{\circ} \mathrm{C} \quad 1$ hour $12 \mathrm{~min}$

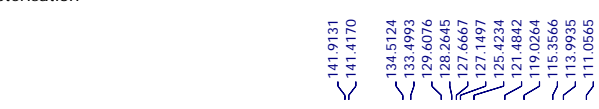<smiles>C=C(c1ccccc1)c1c(C)[nH]c2ccc(Cl)cc12</smiles>

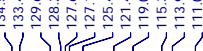

4d

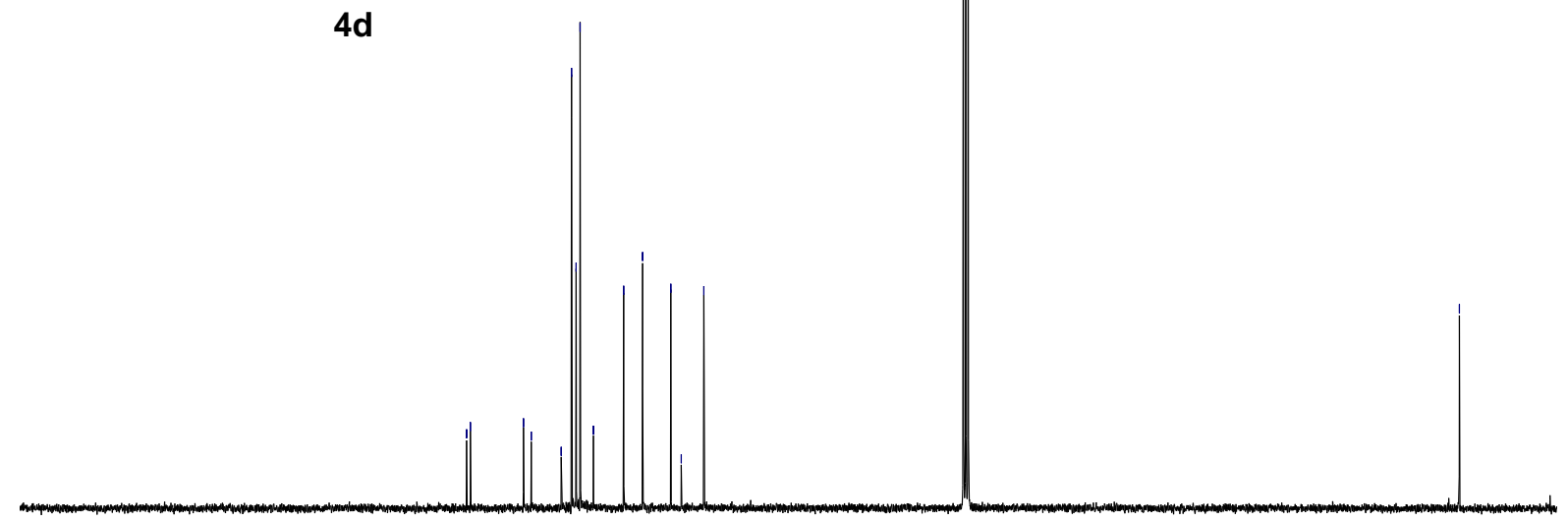

$190 \quad 180 \quad 170$ 
ebmh265char.1.fid

1H 400.1MHz Job 37644 McLean Euan B 265CHAR CDCl3 24.6 C

proton characterisation

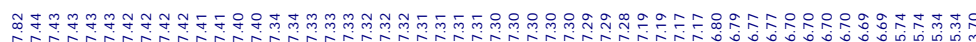
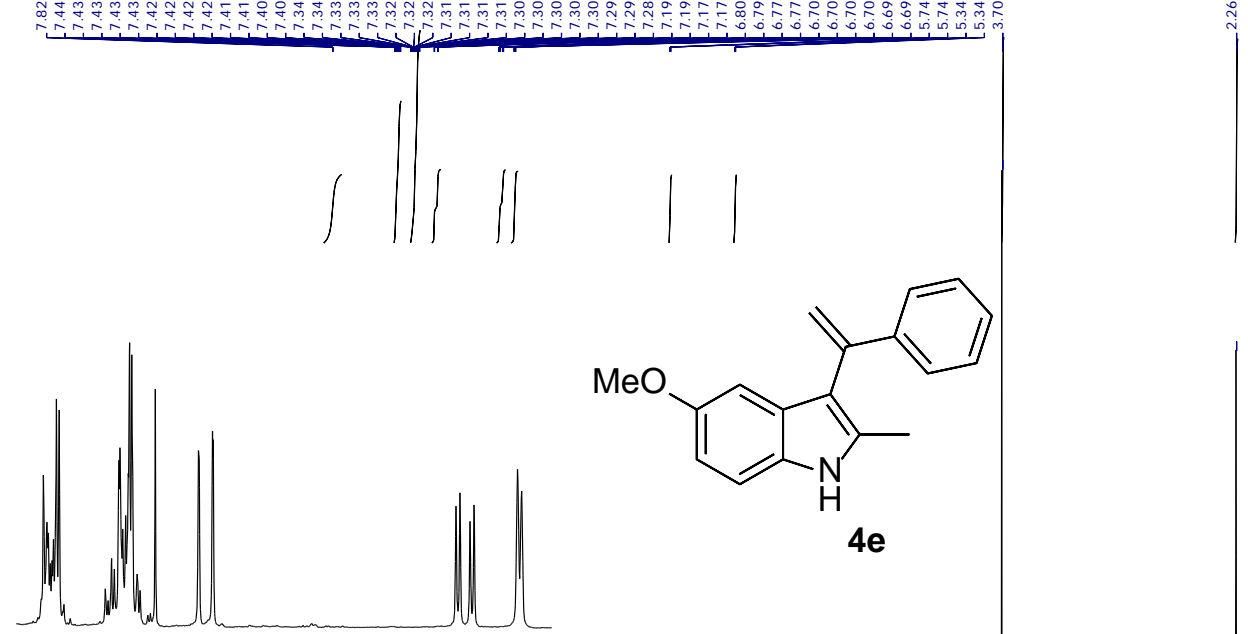

$\mathrm{MeO}$<smiles>C=C(c1ccccc1)c1c(C)[nH]c2ccc(C)cc12</smiles>

$4 e$
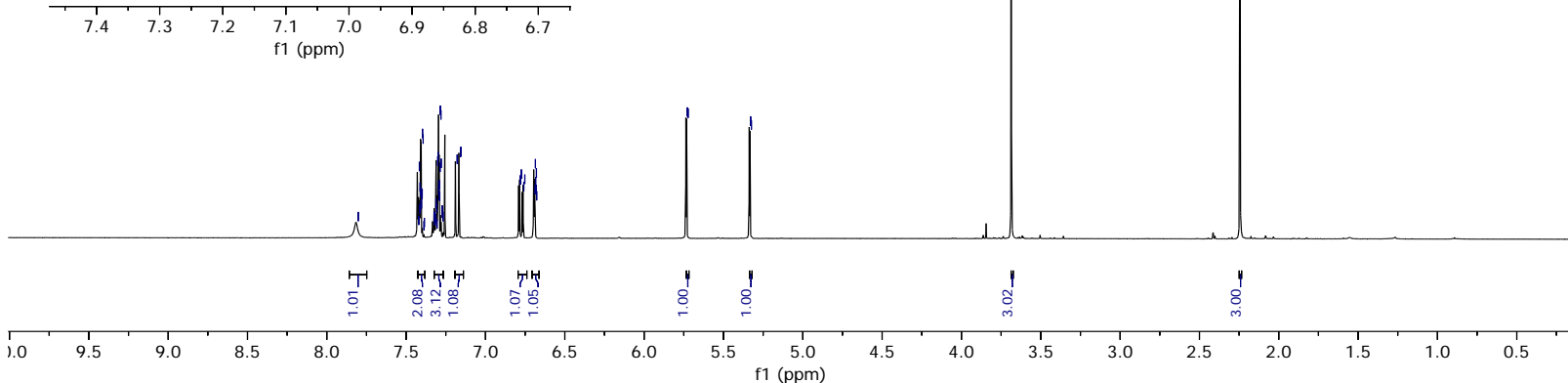

ebmc265char.1.fid

Job 37698 McLean Euan B 265 CHAR CDCl3 $26.0 \curvearrowright \mathrm{C} \quad 1$ hour $12 \mathrm{~min}$ carbon characterisation<smiles>C=C(c1ccccc1)c1c(C)[nH]c2ccc(OC)cc12</smiles>

$4 \mathrm{e}$
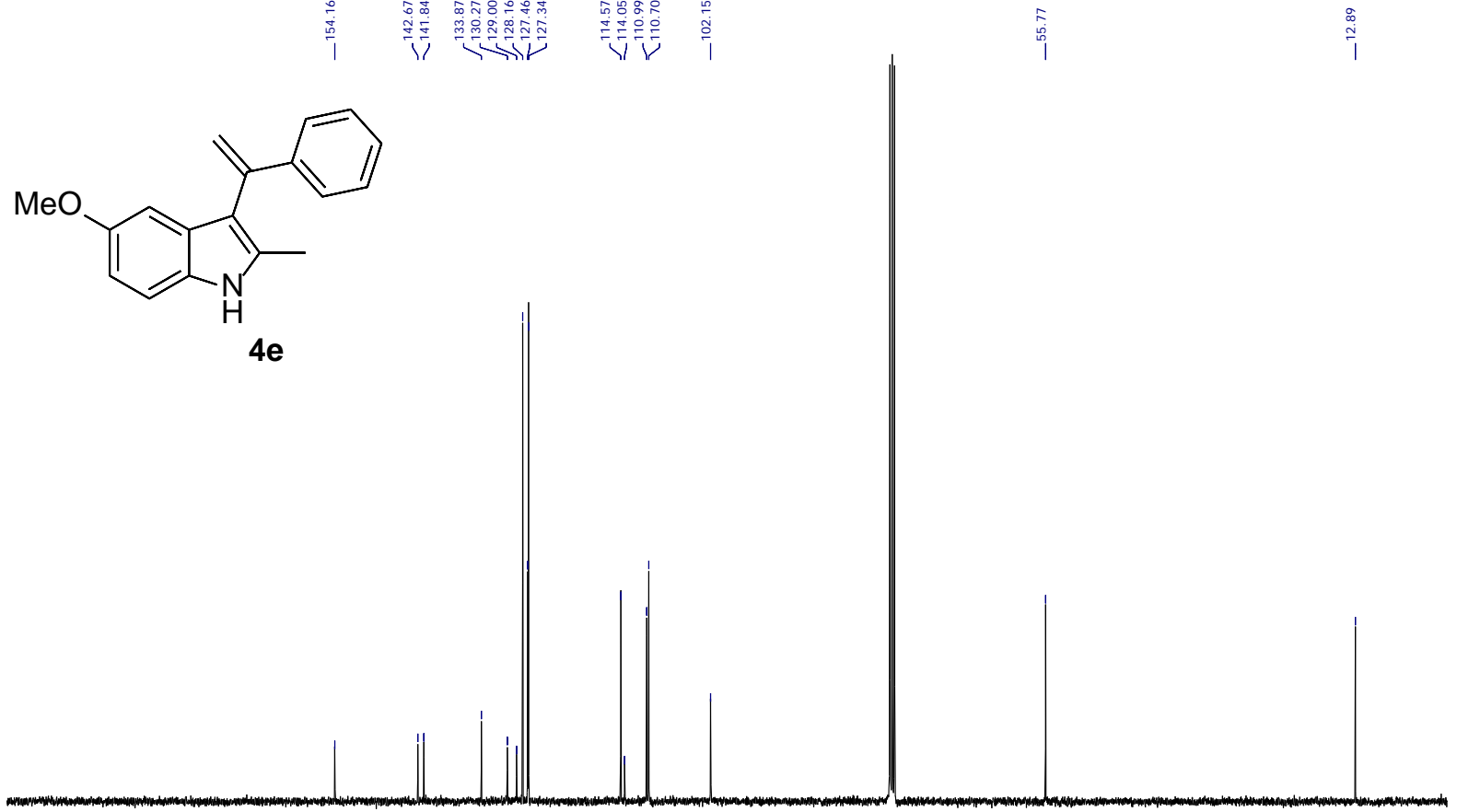

$190 \quad 180+170$

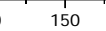

110 


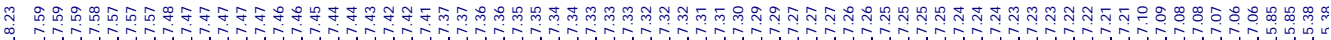

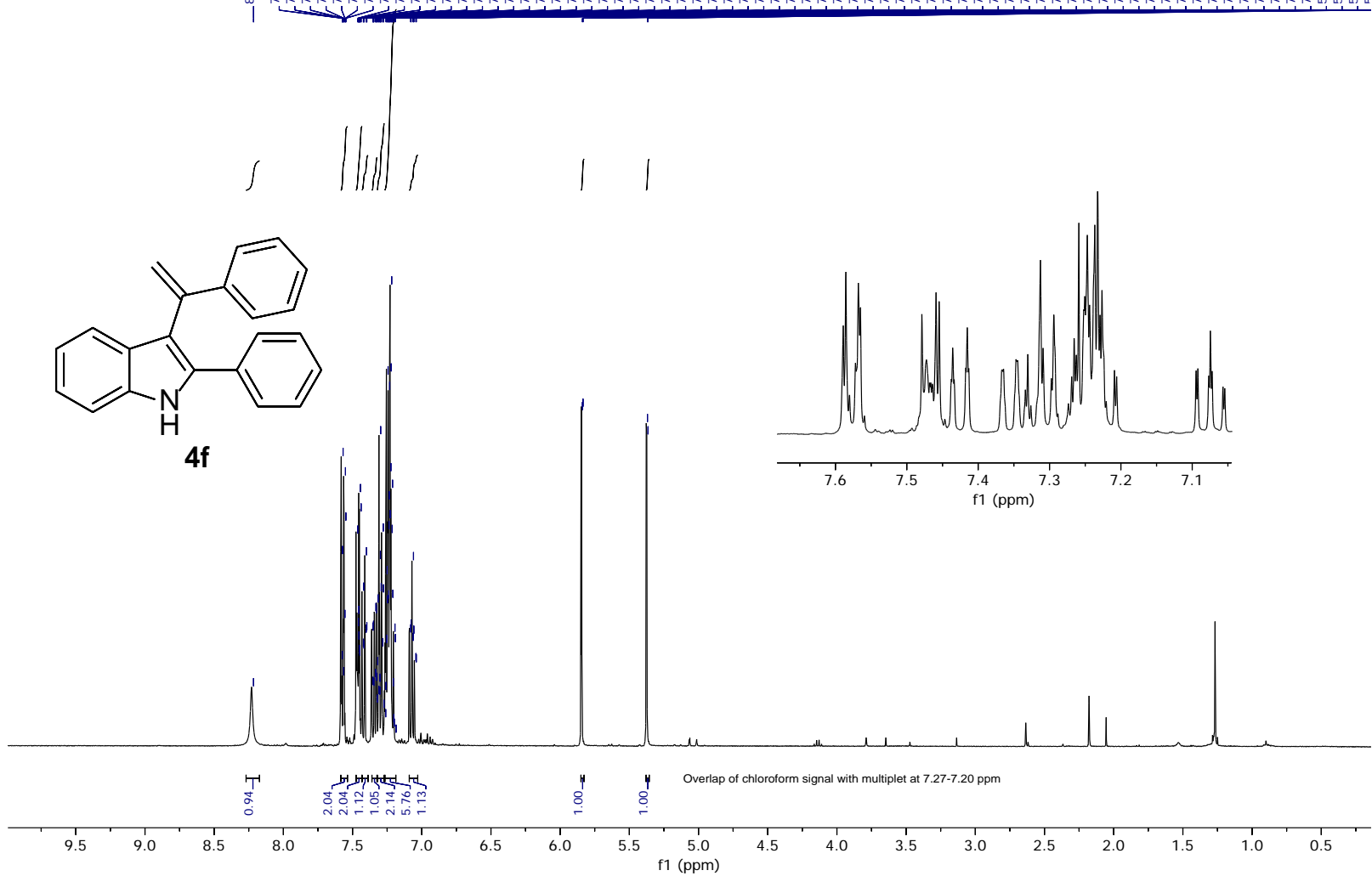

13C 100.6MHz Job 36955 McLean Euan B 220BCHAR CDCl3 $25.5 \oslash$ C 0 hour 58 min carbon characterisation

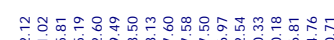

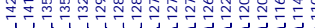<smiles>C=C(c1ccccc1)c1c(-c2ccccc2)[nH]c2ccccc12</smiles>

$4 f$

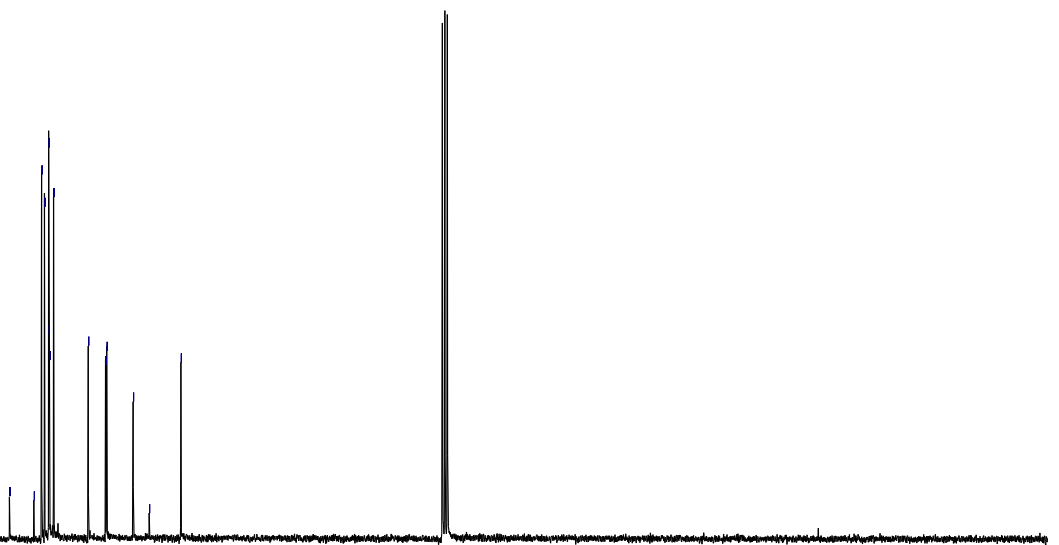

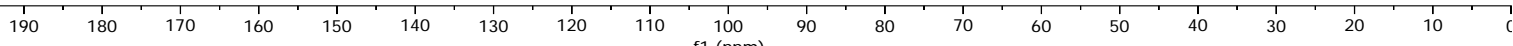


<smiles>C=C(c1ccccc1)c1c(C(C)(C)C)[nH]c2ccccc12</smiles>

49

\section{$\| \int_{i s}$}

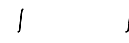

$\begin{array}{llllllllll}7.50 & 7.45 & 7.40 & 7.35 & 7.30 & 7.25 & 7.20 & 7.15 & 7.10 & 7.05\end{array}$ $1(\mathrm{ppm})$

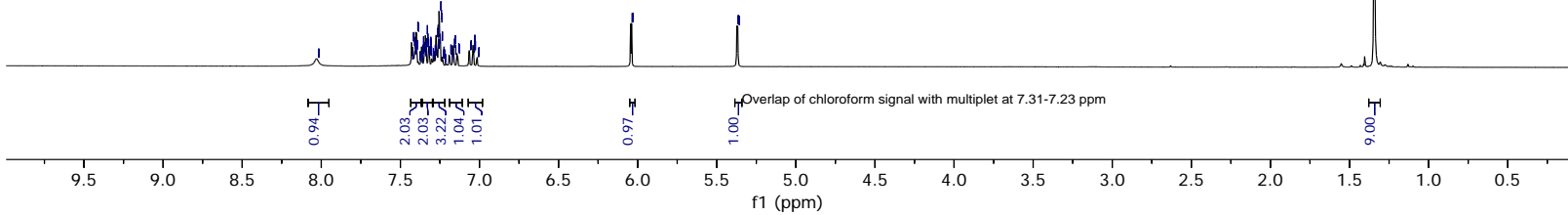

ebmc271char.1.fid

85259 McLean Euan B 271CHAR CDCl3 $22.8 \gg \mathrm{C} \quad 2$ hours $25 \mathrm{~min}$ carbon characterisation
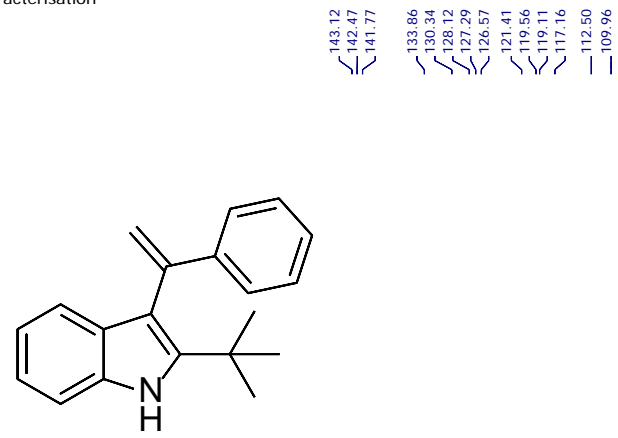

$\mathbf{4 g}$

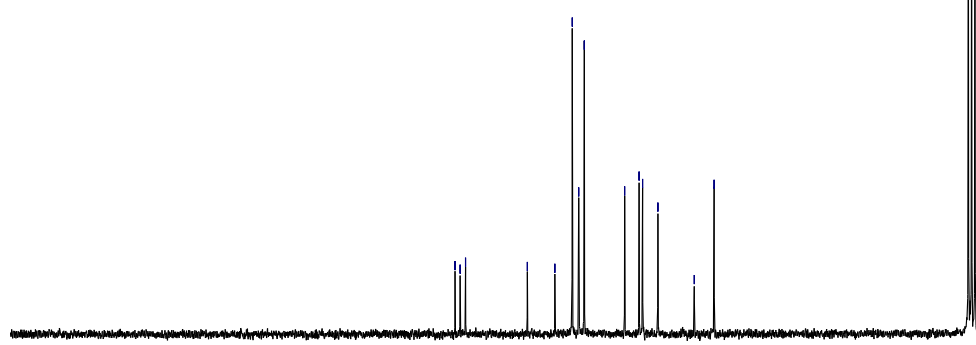

$\begin{array}{ll}190 & 180\end{array}$

160

$150 \quad 140$

130

110 100
f1 $(\mathrm{ppm})$ 


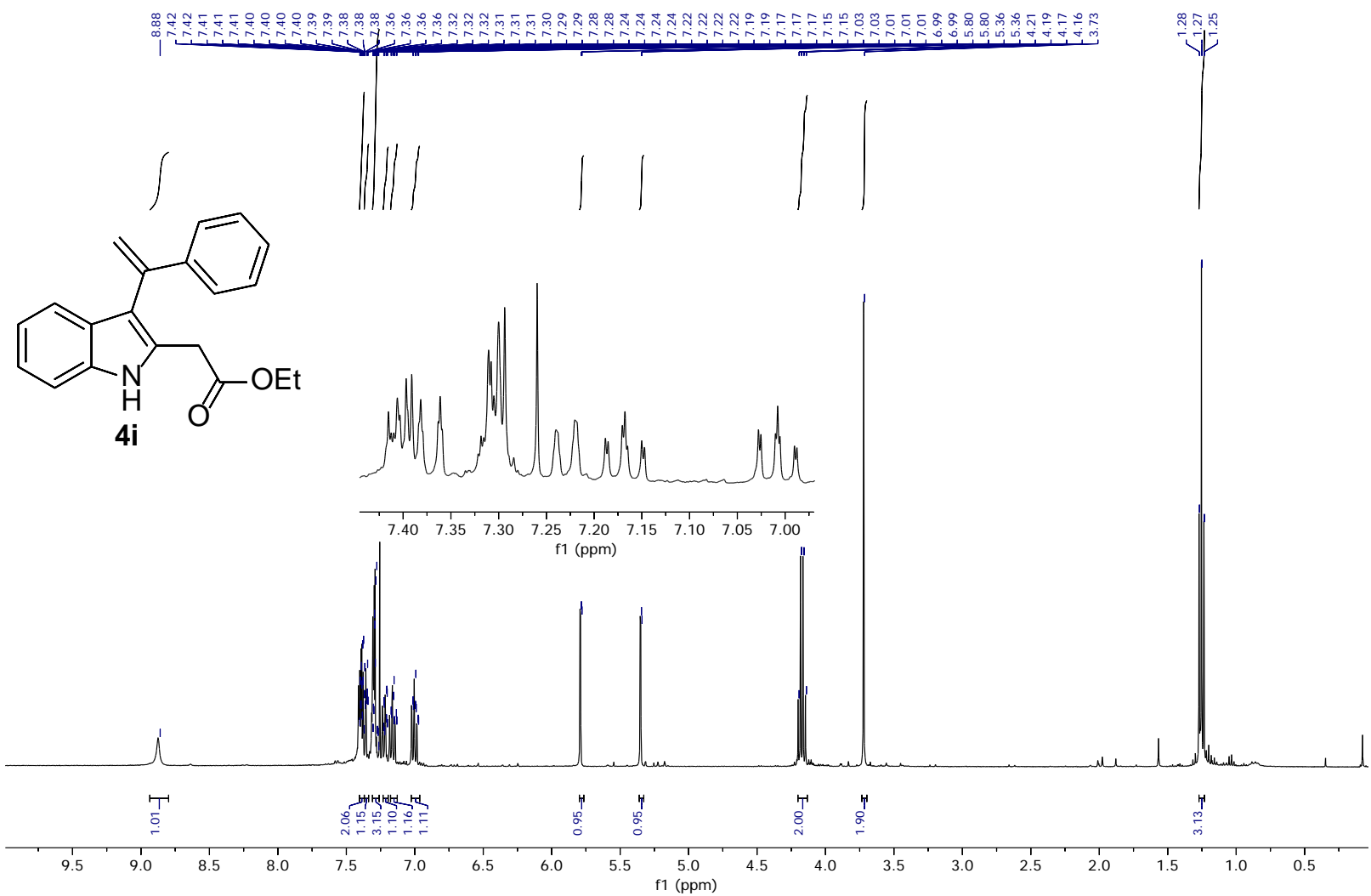

ebmc279char.1.fid

13C 100.6MHz Job 37845 McLean Euan B 279CHAR CDCl3 $26.0 \bigcirc 2$ hours 25 min carbon characterisation<smiles>C=C(c1ccccc1)c1c(CC(=O)OCC)[nH]c2ccccc12</smiles>

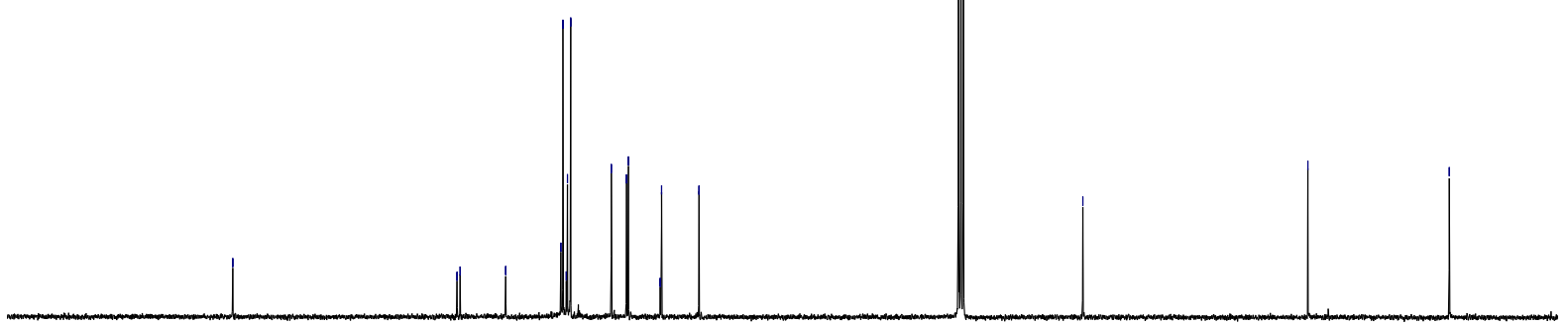


ebmh307char2.1.fid

1H 400.1MHz Job 39210 McLean Euan B 307CHAR2 CDCl3 24.8 $\gg \mathrm{C}$

proton characterisation
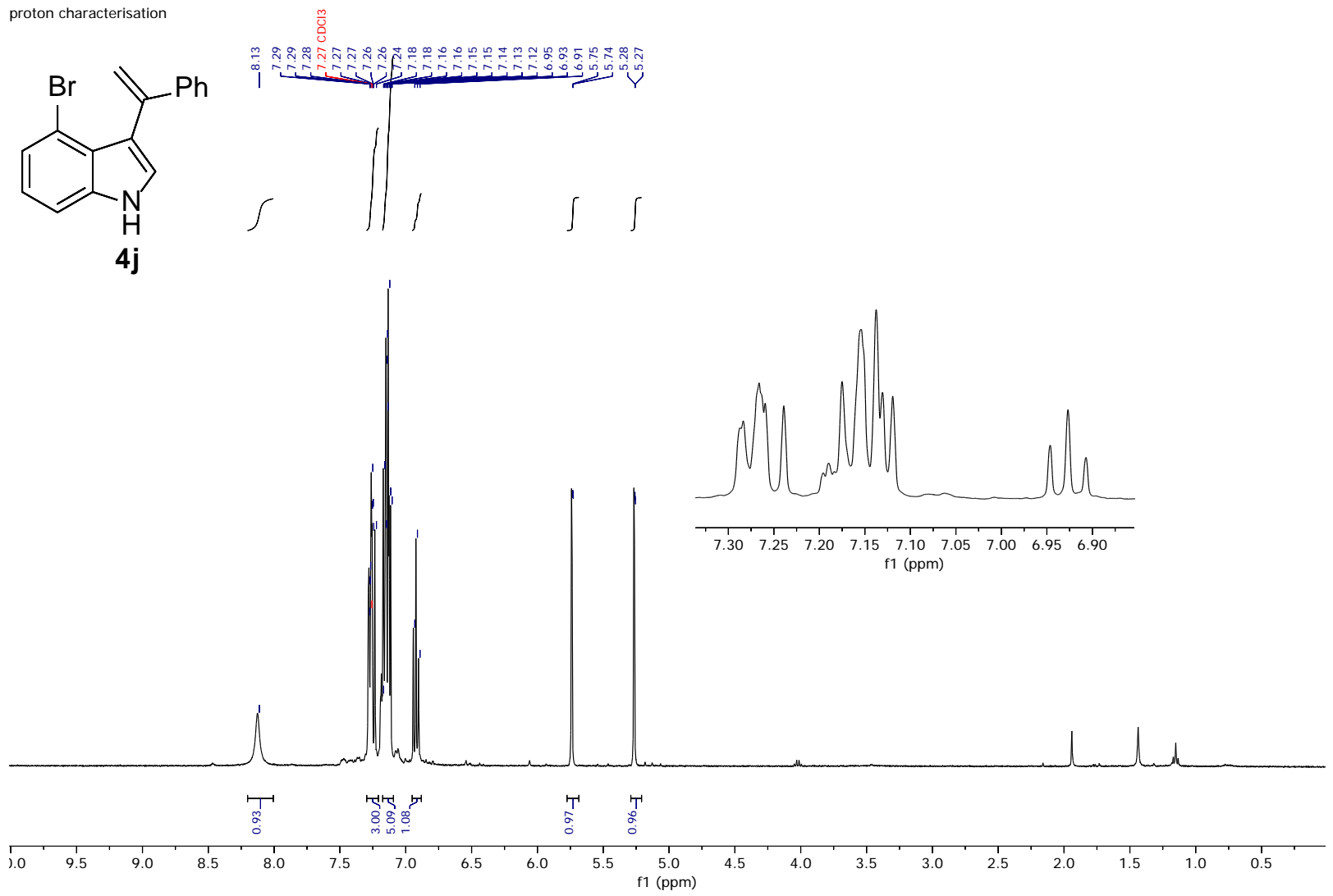

ebmc307char.1.fid

13C 75.5MHz Job 87420 McLean Euan B 307CHAR CDCl3 $25.0 \gg \mathrm{C} \quad 3$ hours 1 min carbon characterisation

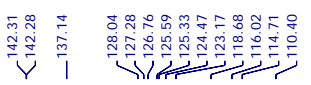
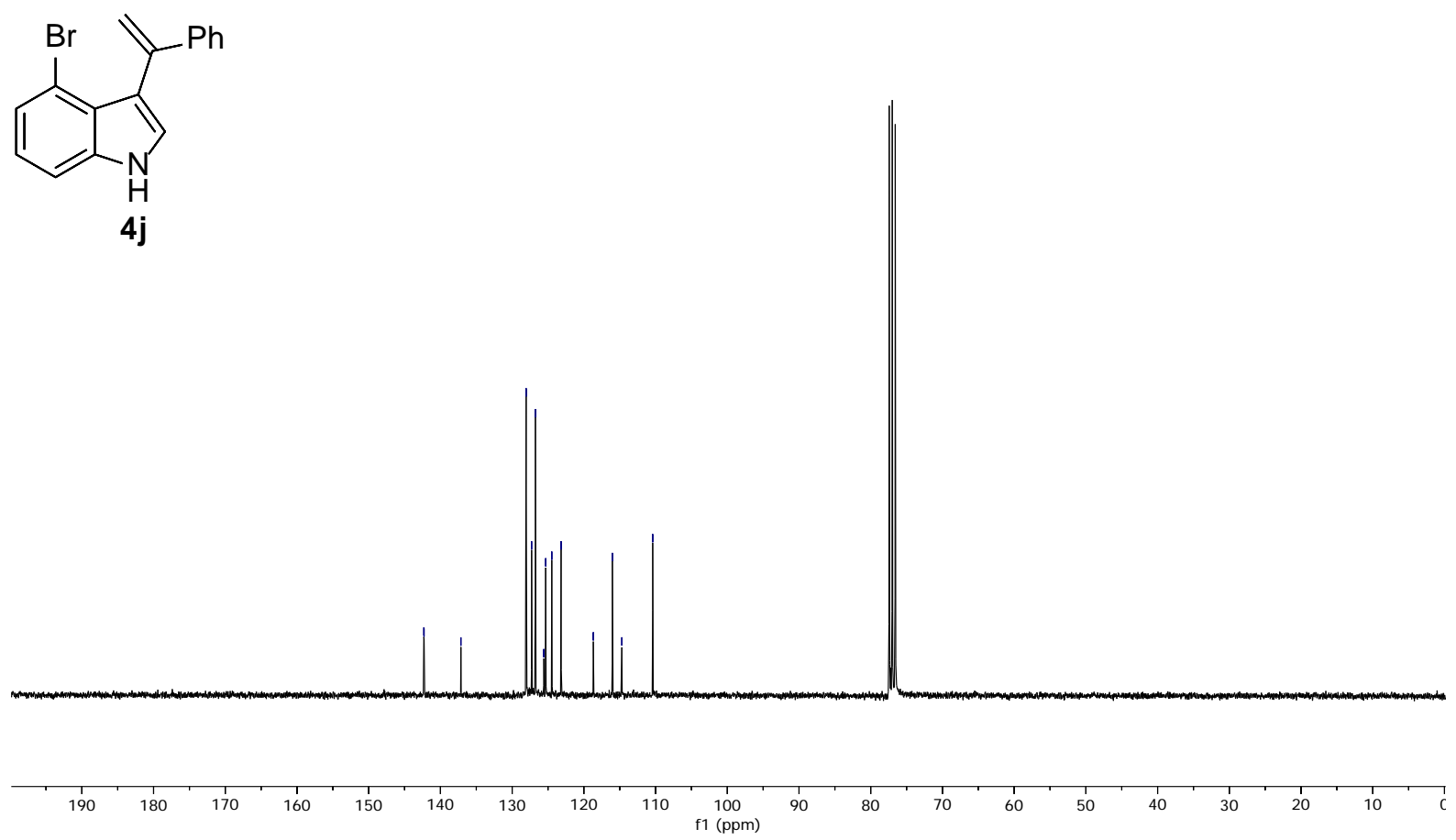

S-38 
ebmh281echar.1.fid

1H 300.1MHz Job 87191 McLean Euan B 281ECHAR CDCl3 25.2〉C

proton characterisation

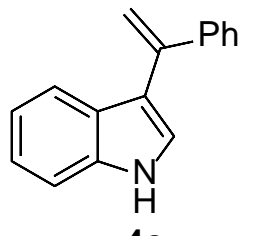

40

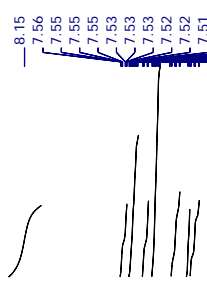

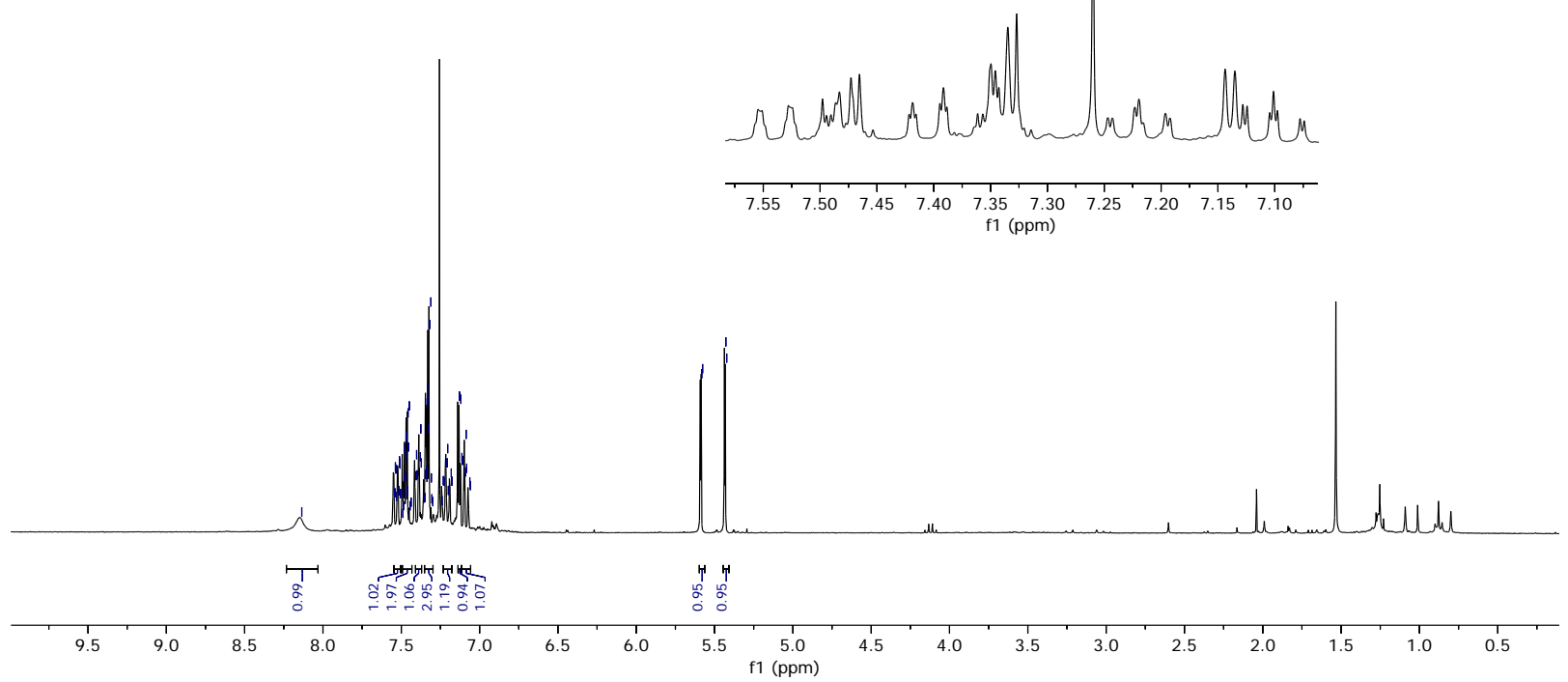

ebmc281echar.1.fid

$13 \mathrm{C} 75.5 \mathrm{MHz}$ Job $87207 \mathrm{McLean}$ Euan B $281 \mathrm{ECHAR} \mathrm{CDCl} 3 \quad 25.0 \gg \mathrm{C} \quad 3$ hours 1 min
carbon characterisation

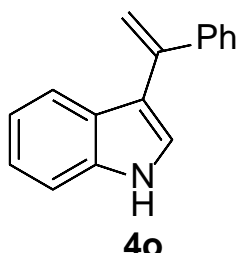

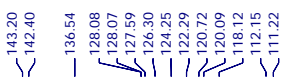

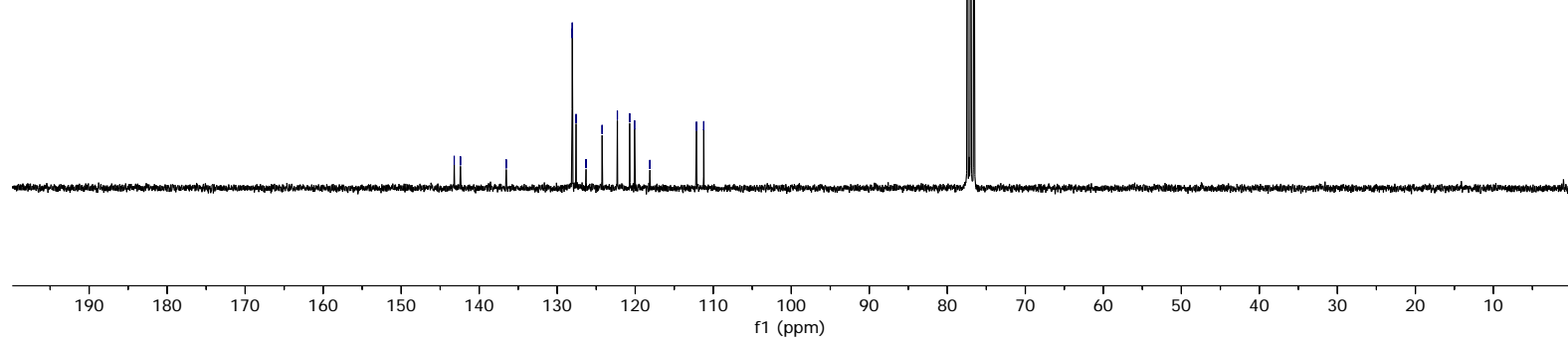

S-39 
ebmh510char.1.fid

1H 400.1MHz Job 41579 McLean Euan B 510CHAR CDCl3 24.9œC

proton characterisation

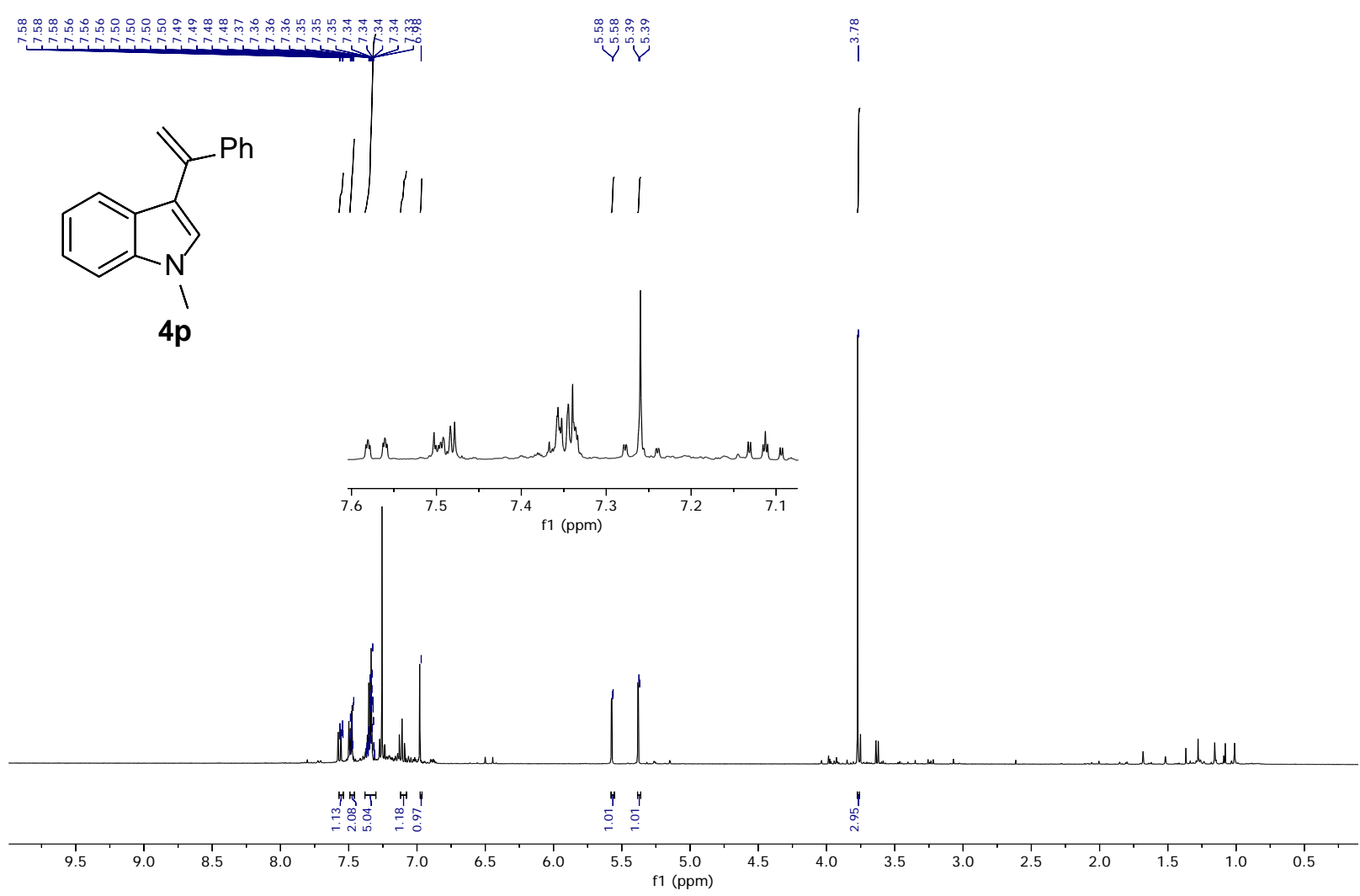

ebmc510char.1.fid

13C 100.6MHz Job 41593 McLean Euan B 510CHAR CDCl3 $26.9 \prec \mathrm{C} \quad 8$ hours 30 min carbon characterisation<smiles>C#Cn1cc(C(=C)c2ccccc2)c2ccccc21</smiles>

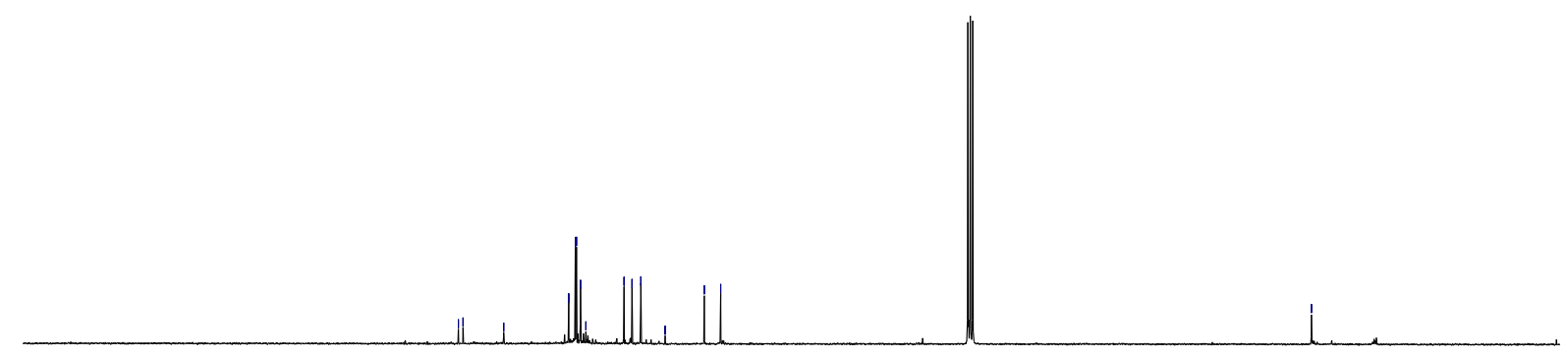

190

$180 \quad 170 \quad 160 \quad 150$

$140 \quad 130$

$120 \quad 110$

100

$90 \quad 80$

80

60

40

$30 \quad 20 \quad 10$ 
ebmh286char.1.fid

1H 400.1MHz Job 38505 McLean Euan B 286CHAR CDCl3 $24.9 \diamond C$

proton characterisation

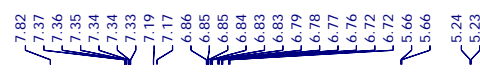
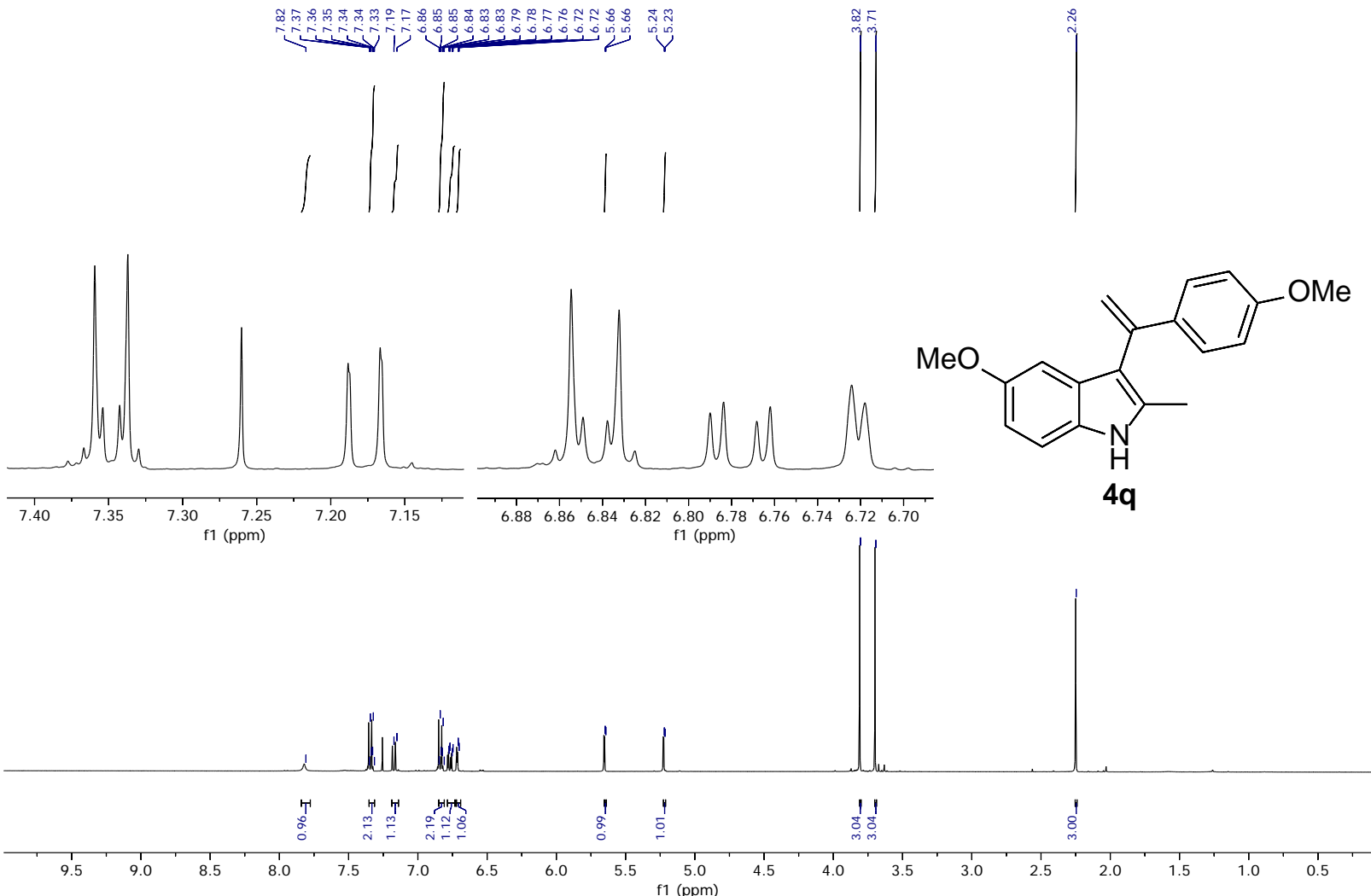

ebmc286char.1.fid

100.6MHz Job 38507 McLean Euan B 286CHAR CDCl3 $27.8 \prec C \quad 1$ hour 27 min carbon characterisation

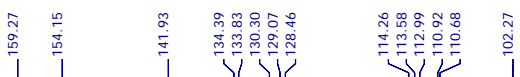

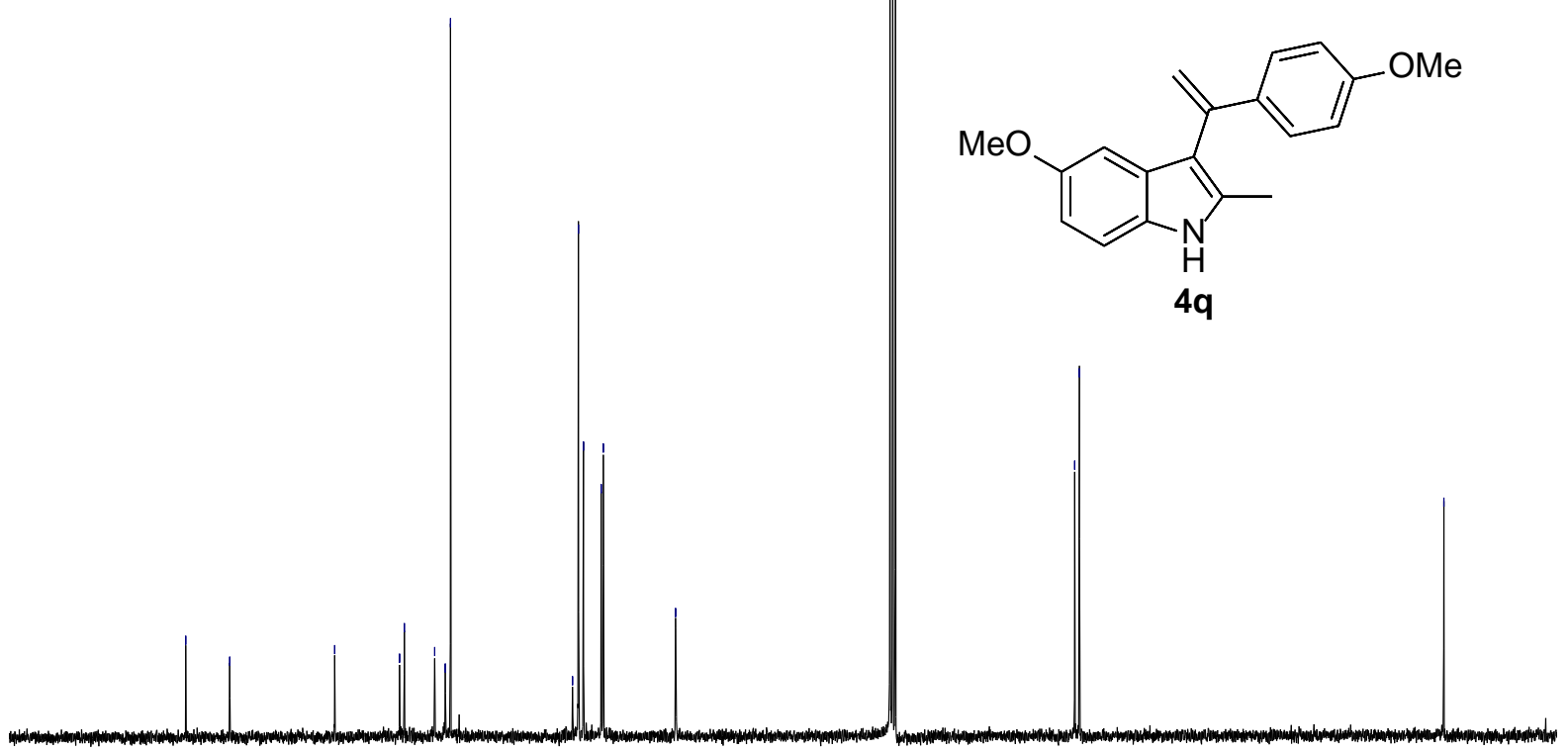

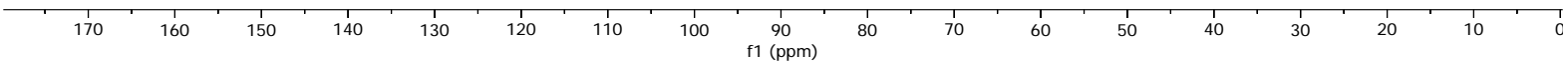


ebmh233char.1.fid

$1 \mathrm{H} 400.1 \mathrm{MHz}$ Job 37019 McLean Euan B 233CHAR CDCl3 24.7 C

proton characterisation

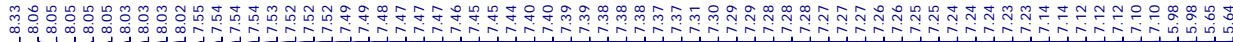

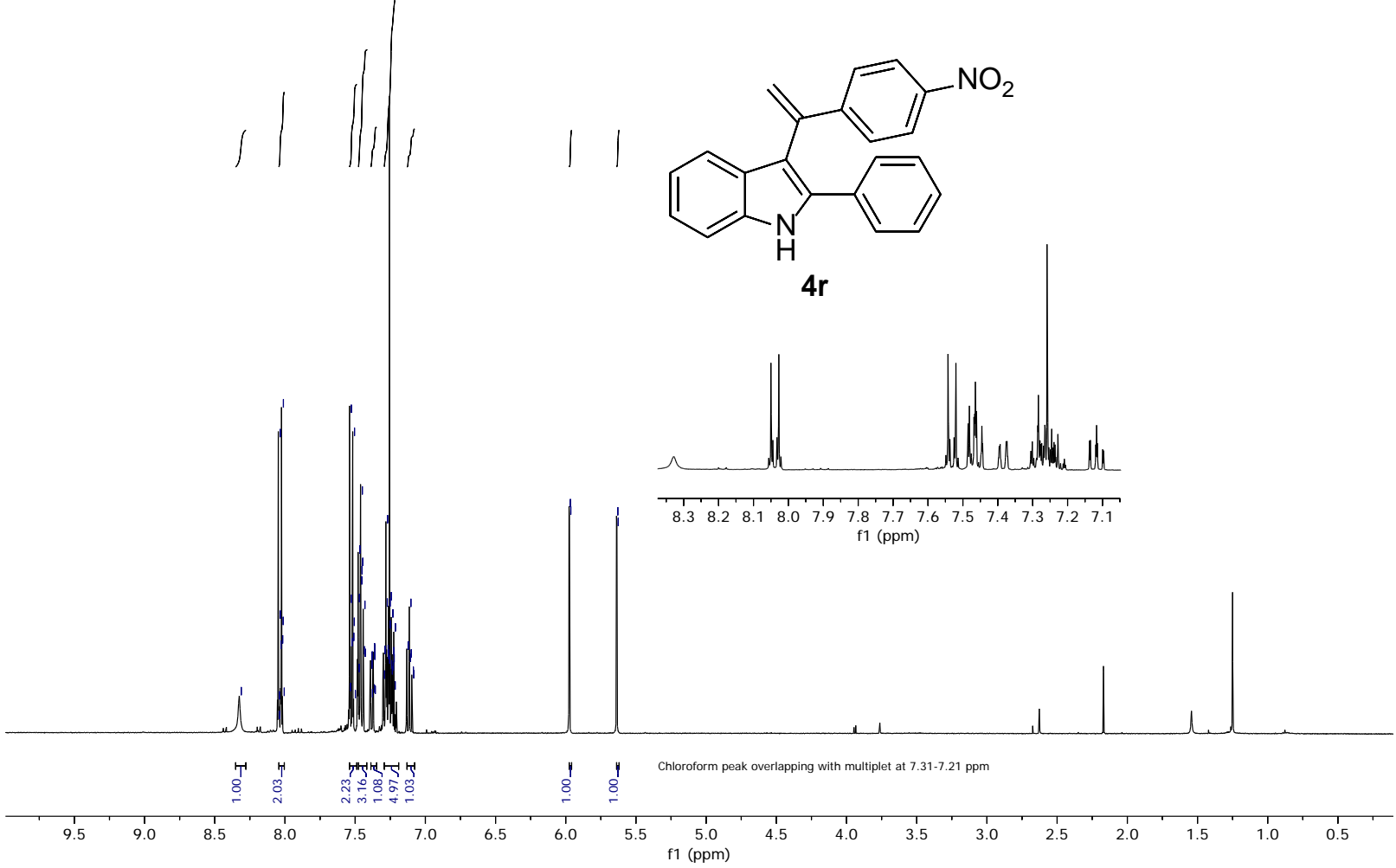

ebmc233char.1.fid

13C 100.6MHz Job 37032 McLean Euan B 233CHAR CDCI3 $25.9 \odot$ C 1 hour 56 min

carbon characterisation

要

$\Rightarrow$ 1

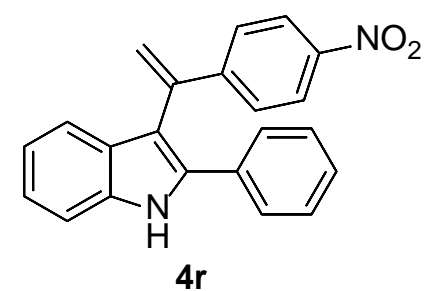

$4 r$

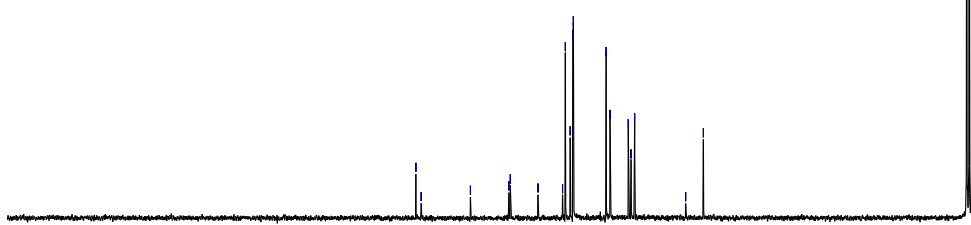

\begin{tabular}{lllllllllllllllllllll}
\hline & 190 & 180 & 170 & 160 & 150 & 140 & 130 & 120 & 110 & 100 & 90 & 80 & 70 & 60 & 50 & 40 & 30 & 20 & 10 & $(10$
\end{tabular} 


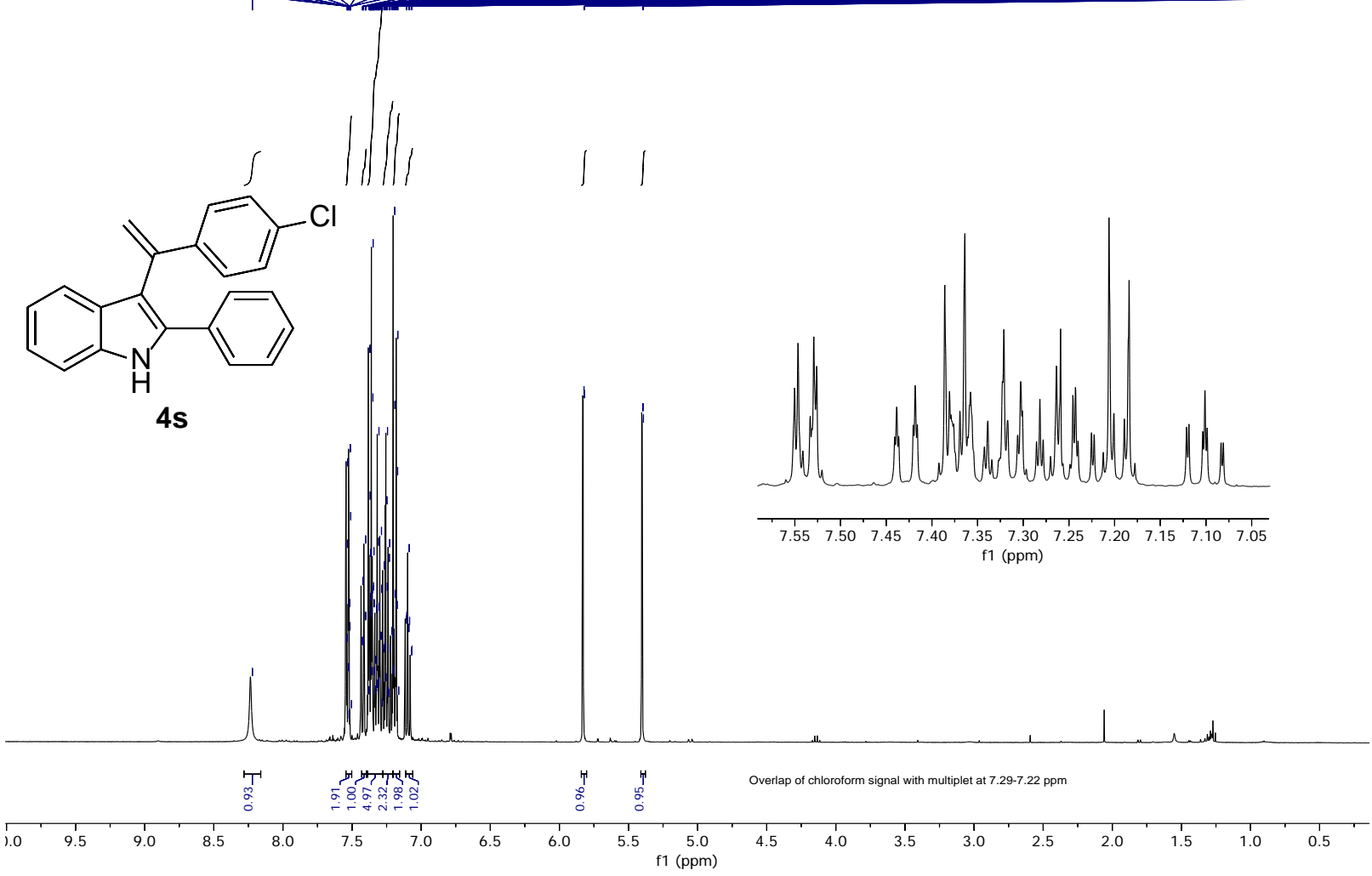

ebmc260char.1.fid

13C 100.6MHz Job 37483 McLean Euan B 260CHAR CDCl3 $26.1 \odot C \quad 0$ hour 29 min carbon characterisation

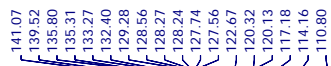

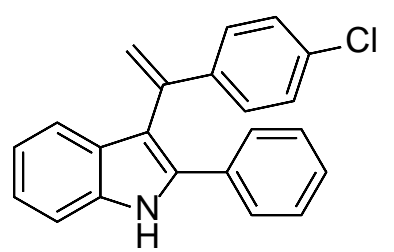

$4 s$

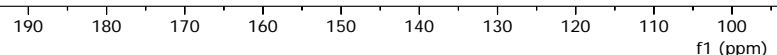




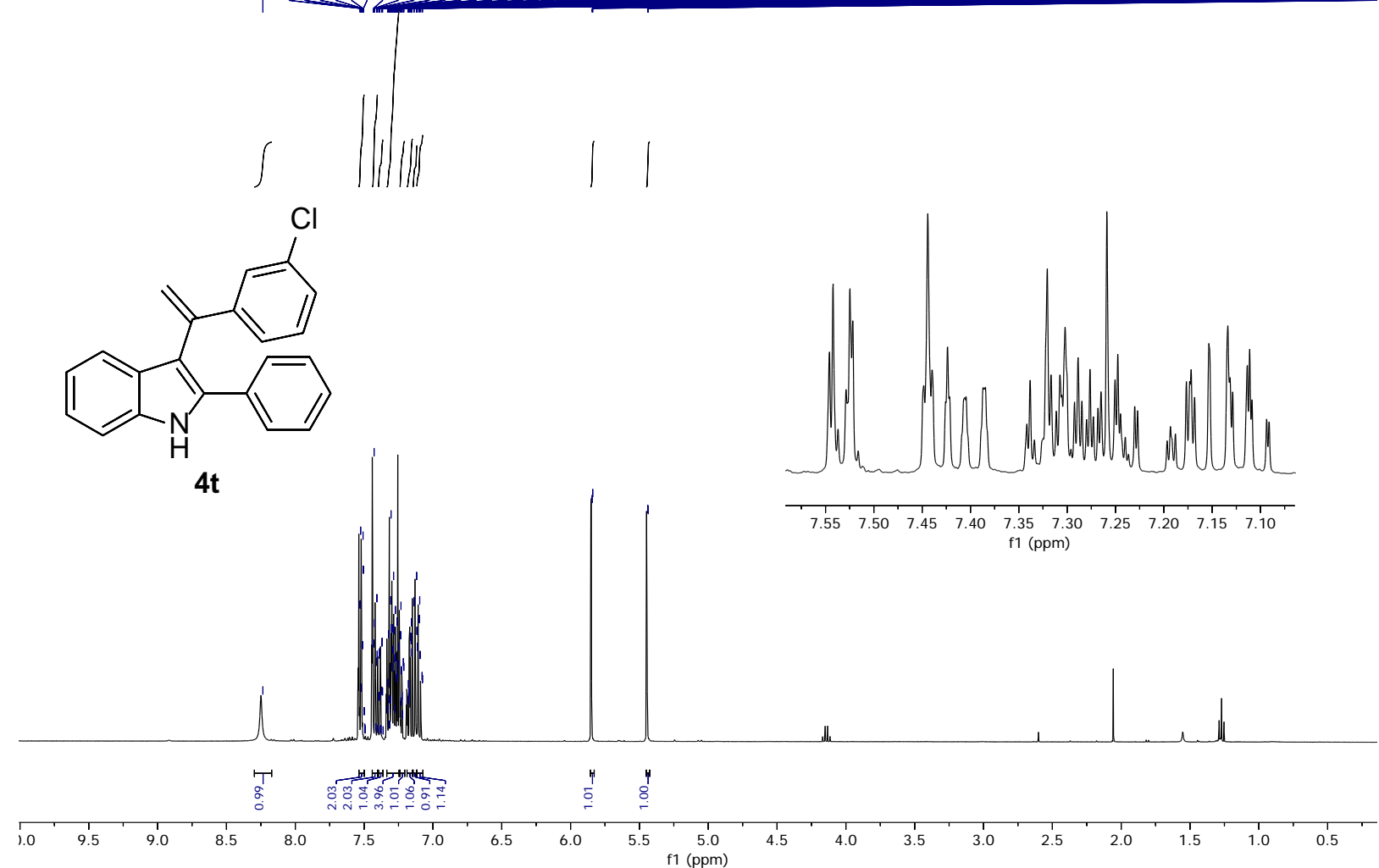

ebmc262char.1.fid

13C 100.6MHz Job 37484 McLean Euan B 262CHAR CDCl3 $26.1 \diamond \mathrm{C} \quad 0$ hour 43 min carbon characterisation

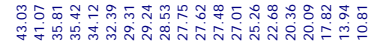 \\ 11}<smiles>C=C(c1cccc(Cl)c1)c1c(-c2ccccc2)[nH]c2ccccc12</smiles>

4t

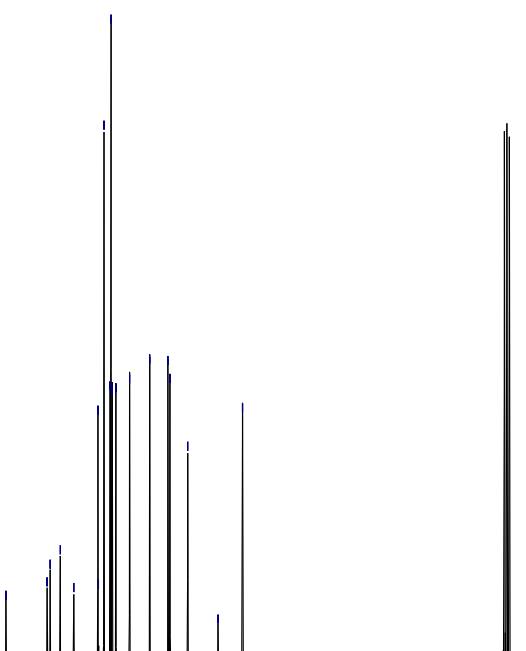




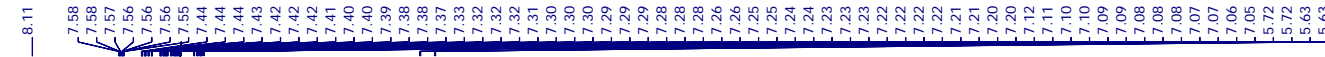

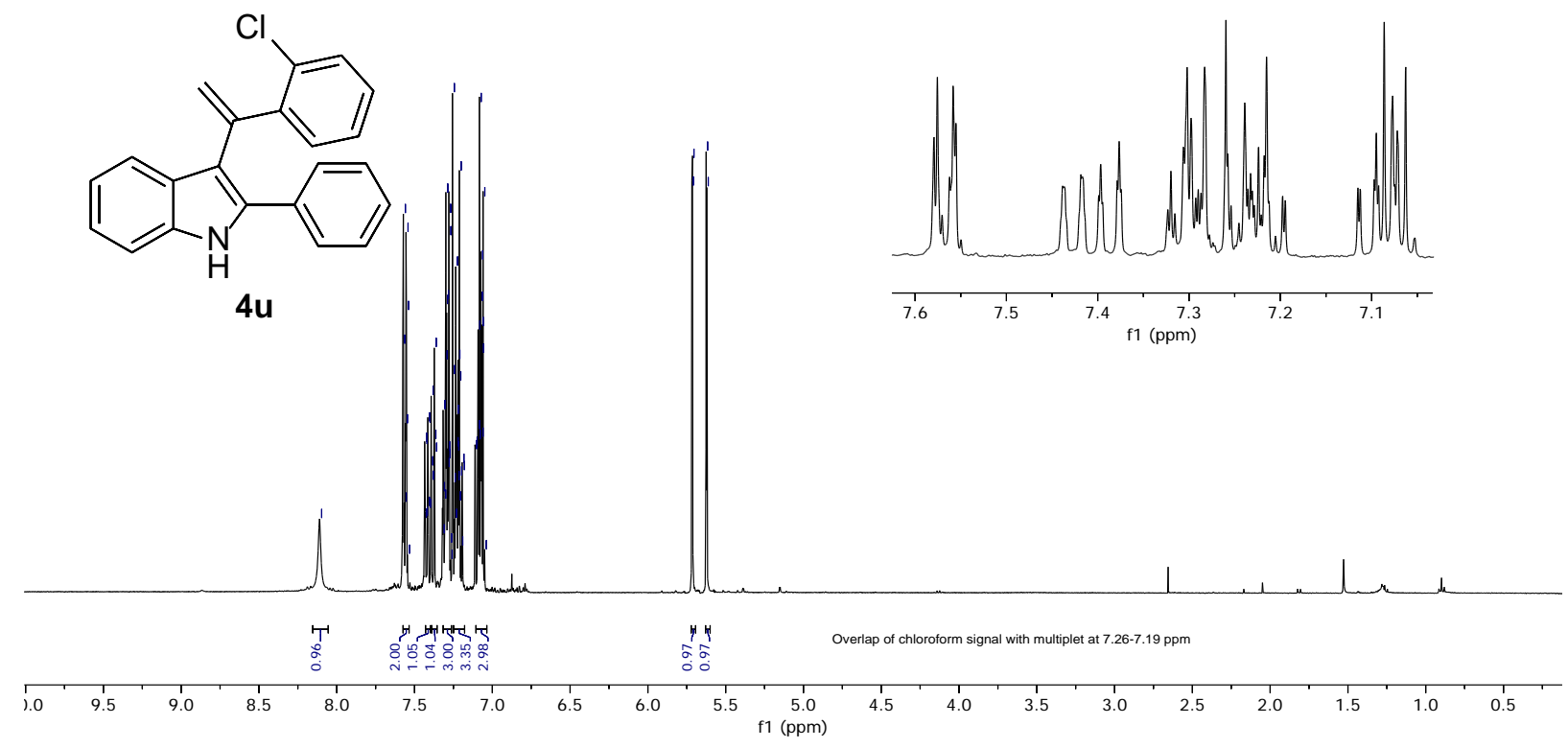

ebmc276char.1.fid

13C 100.6MHz Job 37700 McLean Euan B 276CHAR CDCI3 $25.9 \odot \mathrm{C} \quad 0$ hour $58 \mathrm{~min}$ carbon characterisatio

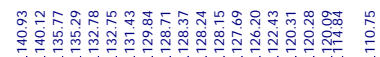<smiles>C=C(c1ccccc1Cl)c1c(-c2ccccc2)[nH]c2ccccc12</smiles>

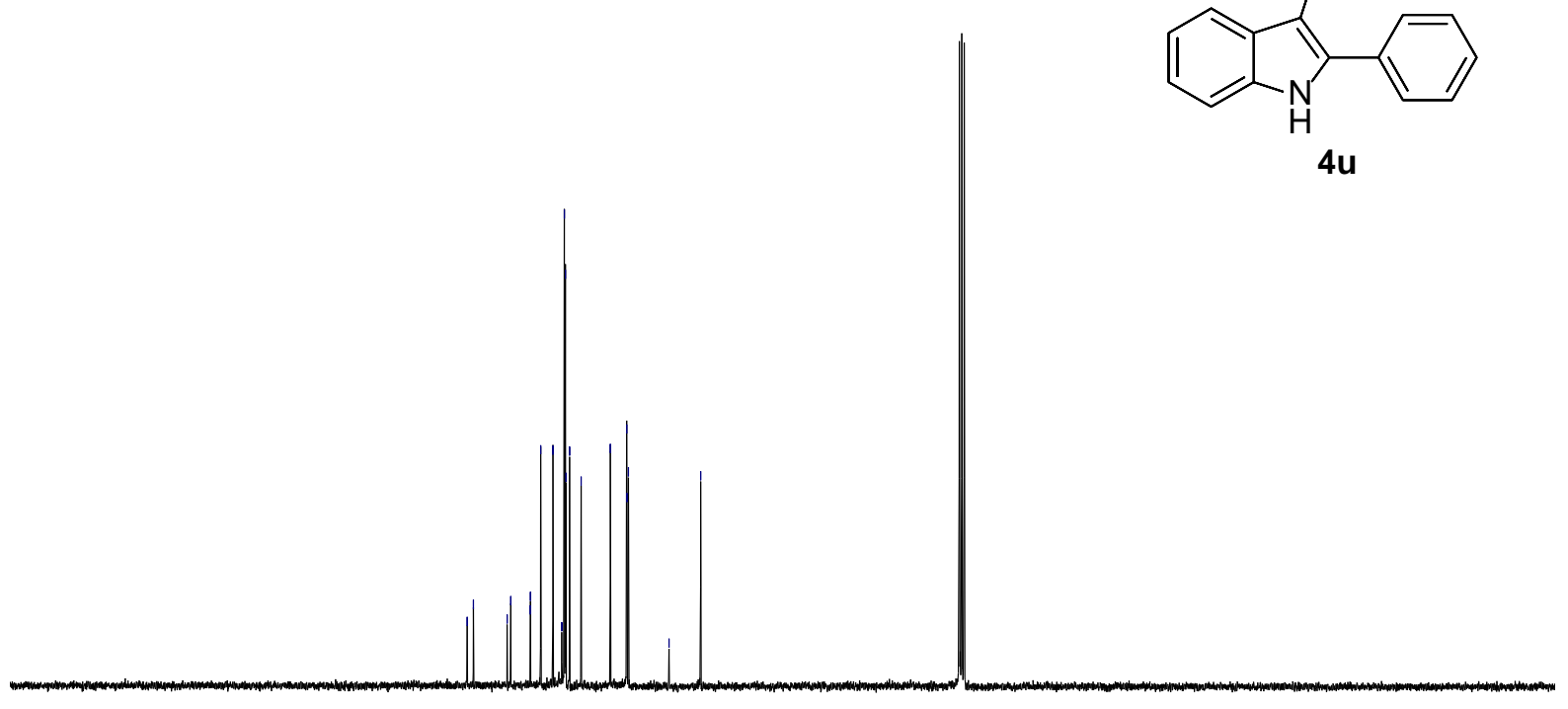

$190-180-170$

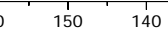

$130 \quad 120$ 
ebmh288char.1.fid

1H 300.1MHz Job 86966 McLean Euan B 288CHAR CDCl3 25.1仓C

proton characterisation

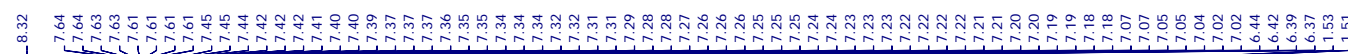<smiles>C/C=C(\c1ccccc1)c1c(-c2ccccc2)[nH]c2ccccc12</smiles>

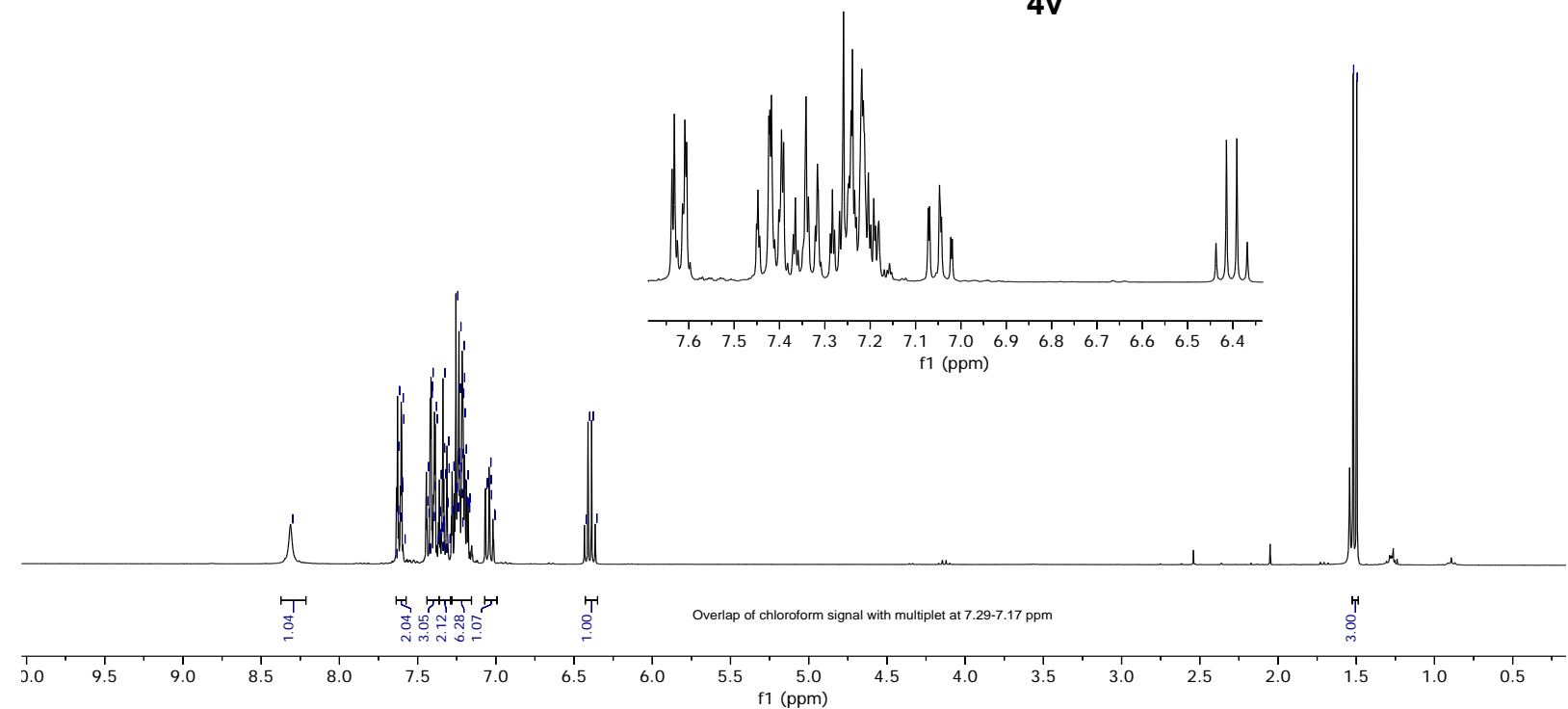

4v

ebmc288char.1.fid

13C 75.5MHz Job 86989 McLean Euan B 288CHAR CDCl3 $25.1 \diamond \mathrm{C} \quad 2$ hours 25 min carbon characterisation<smiles>C/C=C(\c1ccccc1)c1c(-c2ccccc2)[nH]c2ccccc12</smiles>

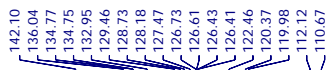

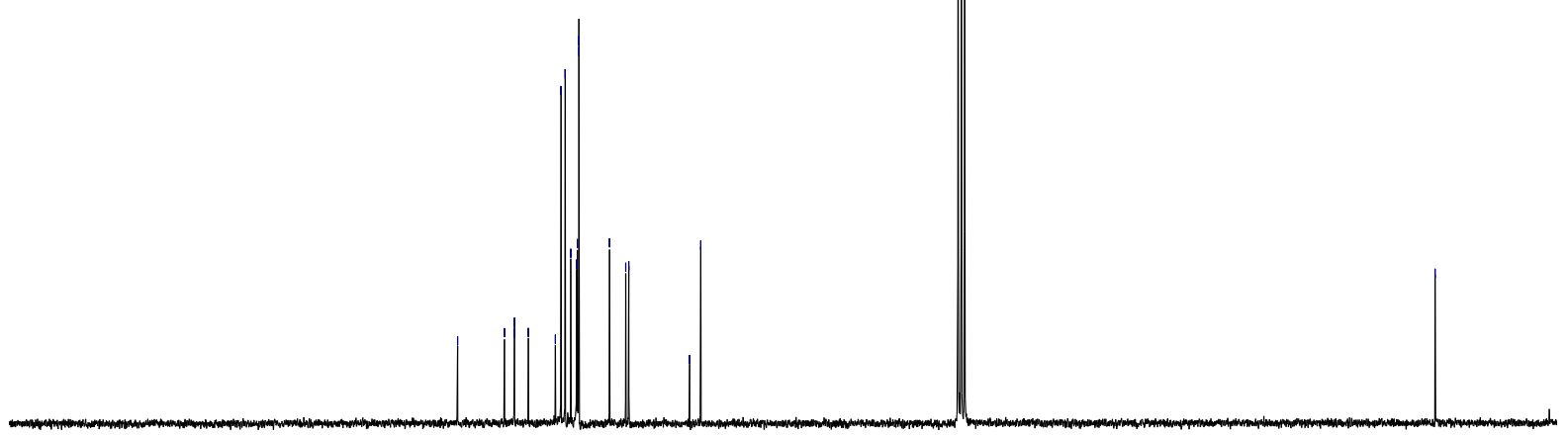

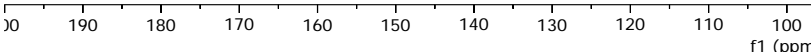

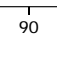

80

60 

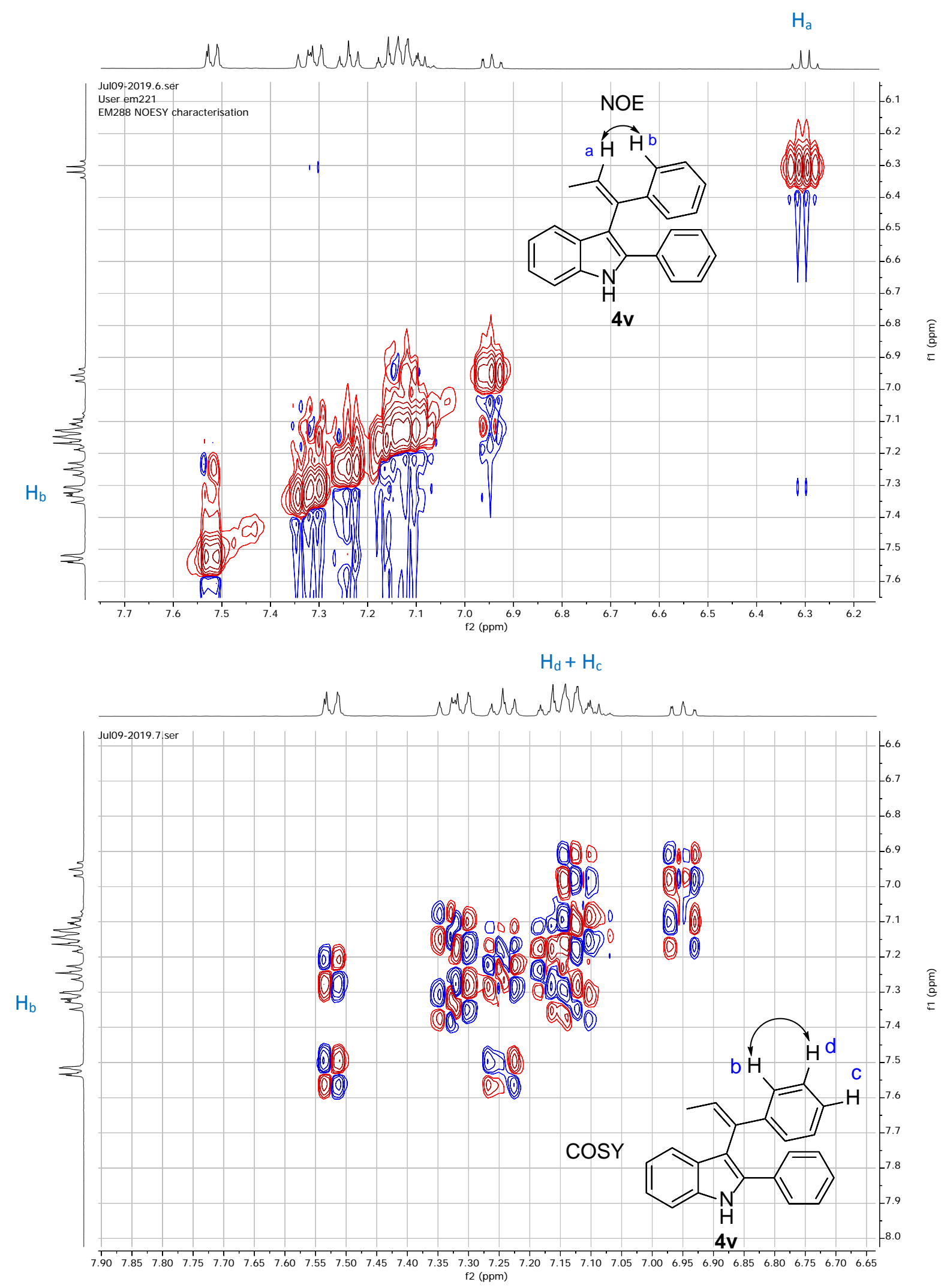

${ }^{1} \mathrm{H}$ NMR $\left(400 \mathrm{MHz}, \mathrm{CDCl}_{3}\right)$ 


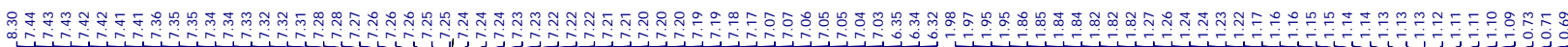

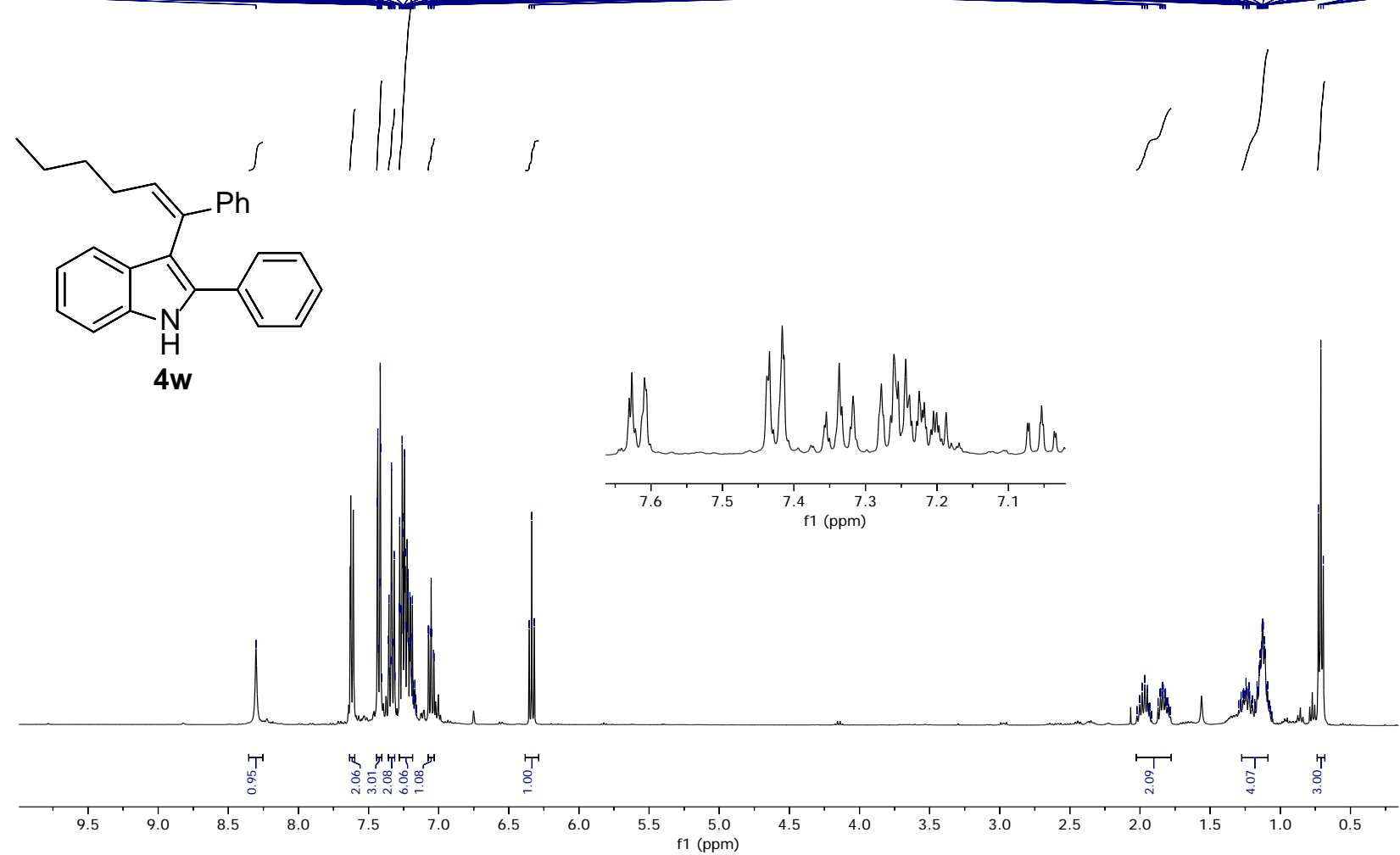

${ }^{13} \mathrm{C}$ NMR (101 $\left.\mathrm{MHz}, \mathrm{CDCl}_{3}\right)$

Jul05-2019.5.fid
User em221

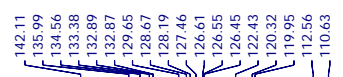
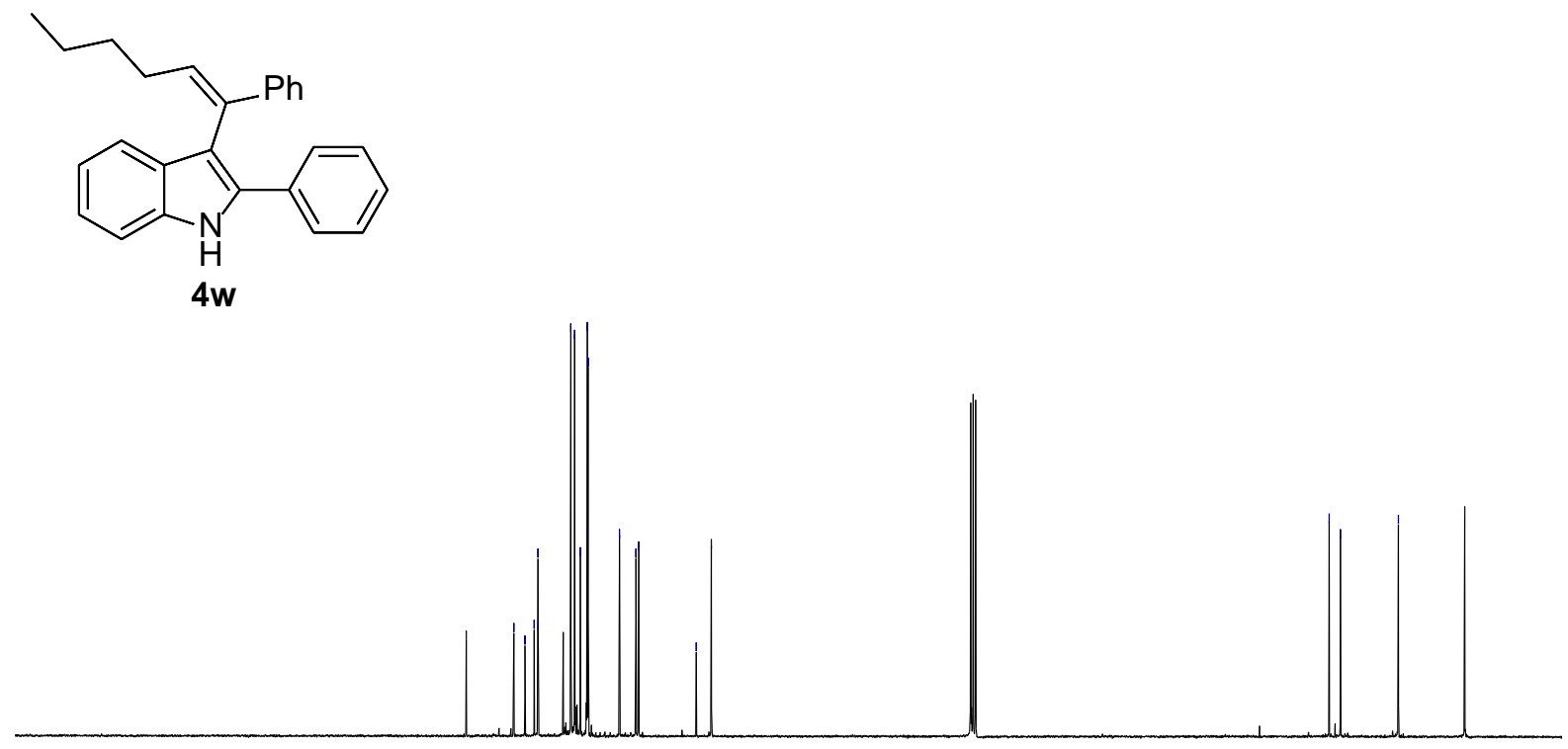

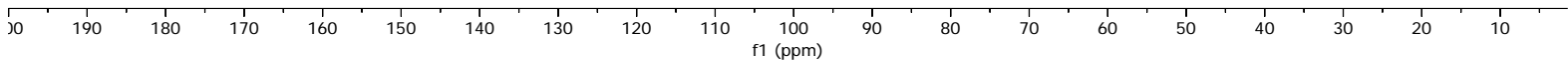




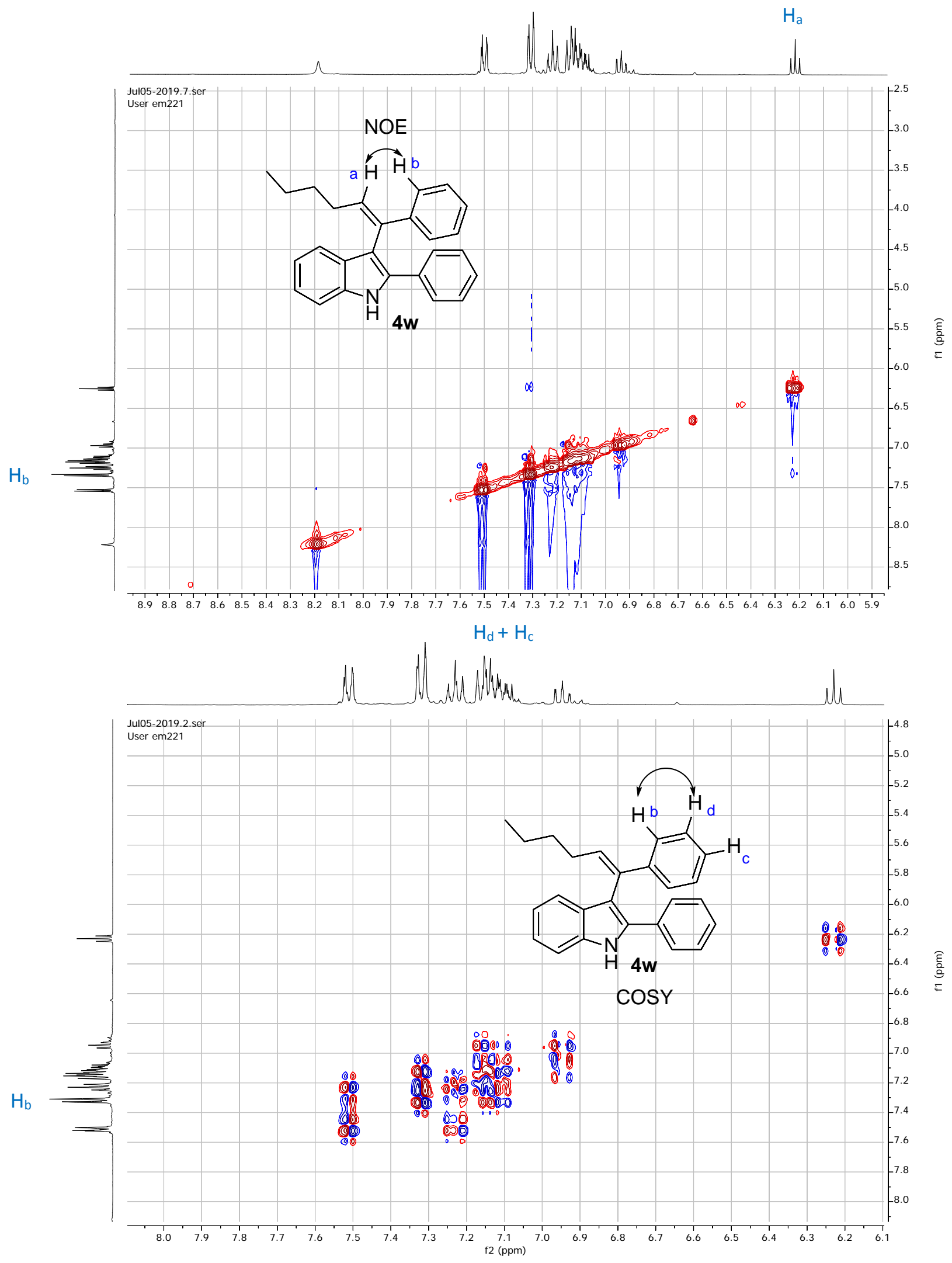




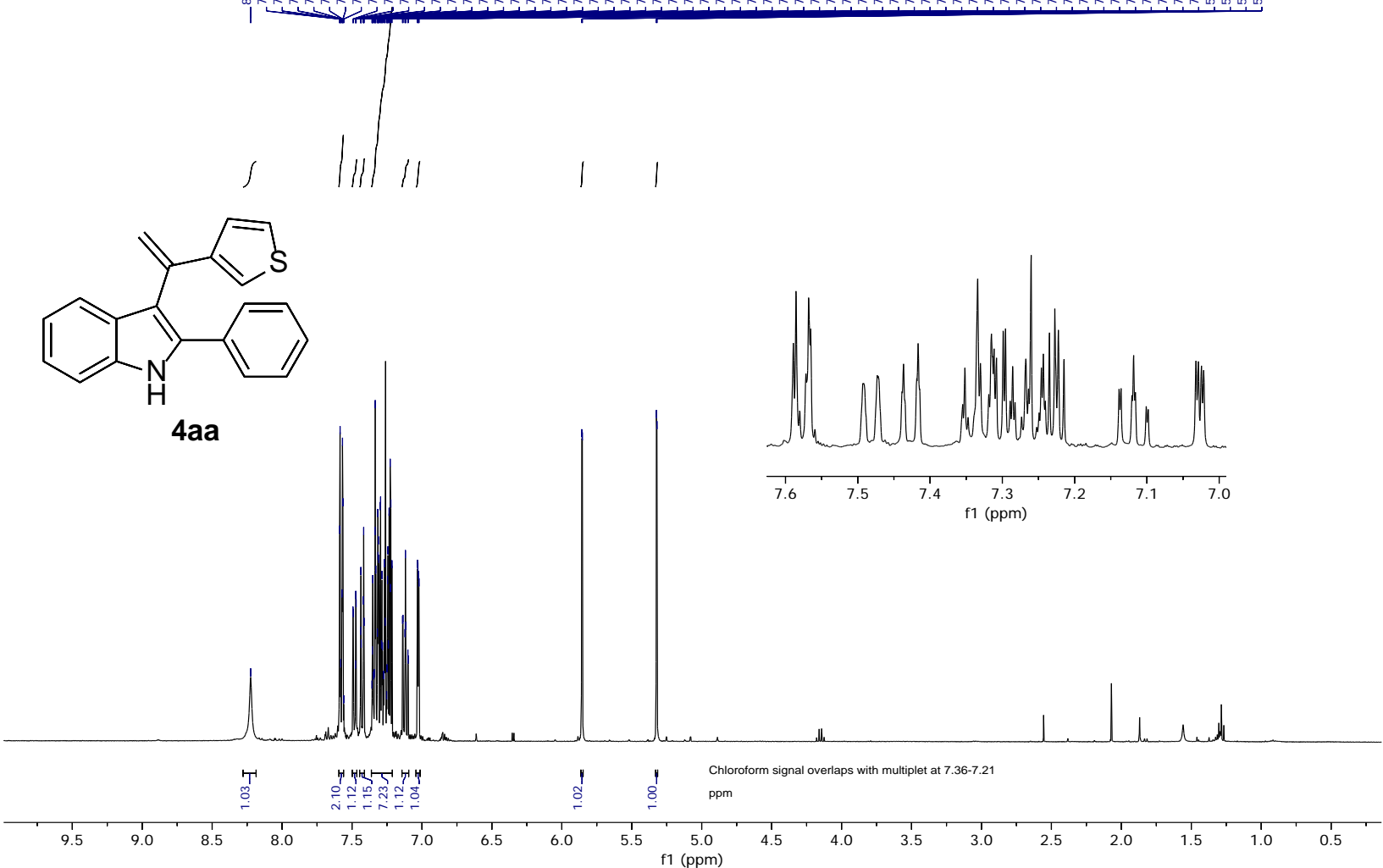

ebmc264char.1.fid

(3C 100.6MHz Job 37485 McLean Euan B 264CHAR CDCl3 $25.7 \circlearrowright C \quad 0$ hour 58 min carbon characterisation

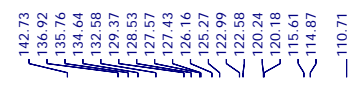

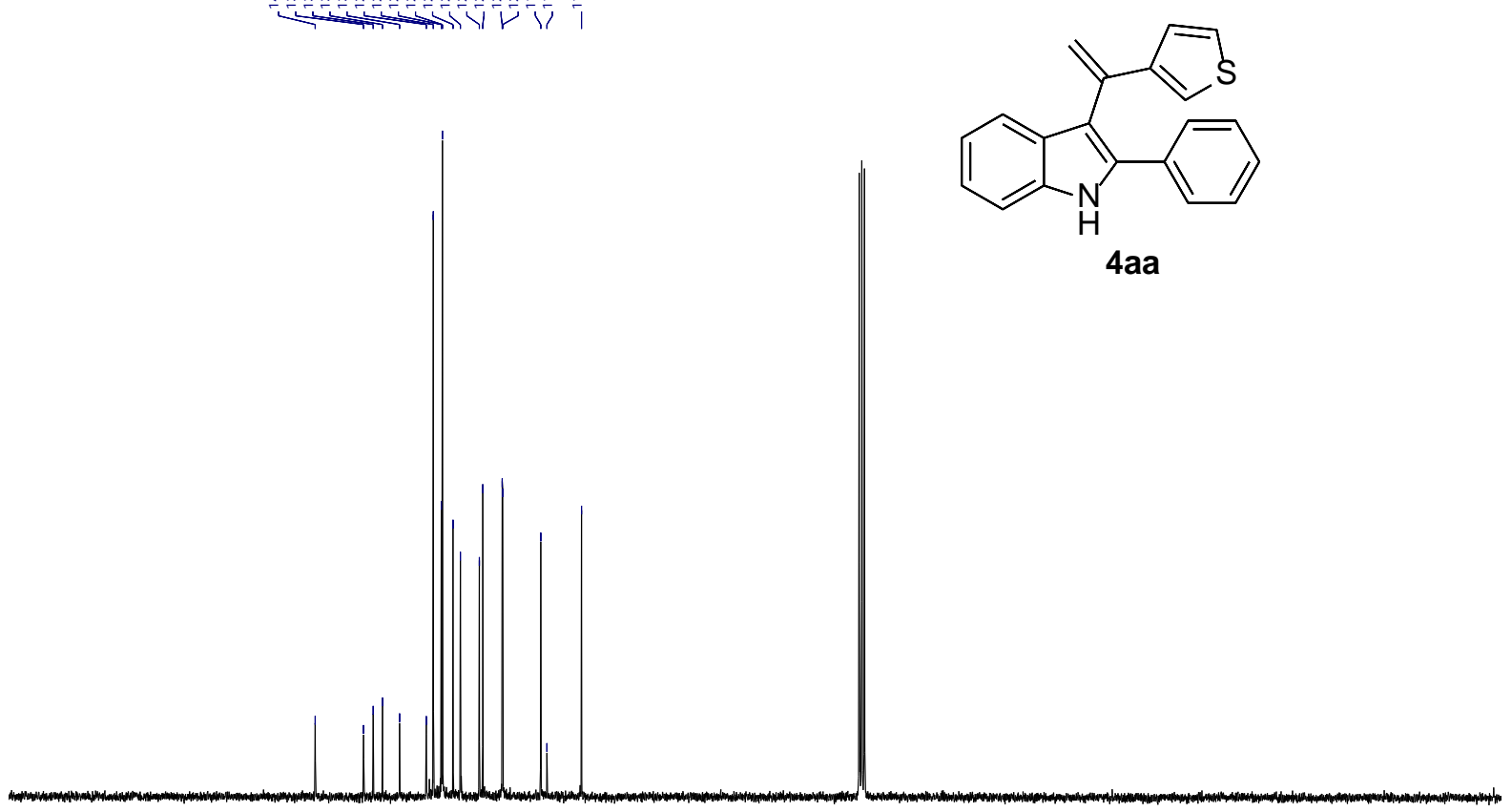

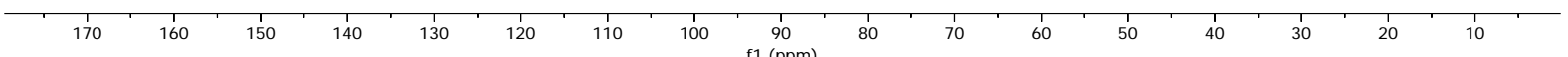


1H 300.1MHz Job 87558 McLean Euan B 314CHAR2 CDCl3 25.1 C

proton characterisation

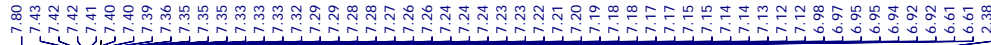

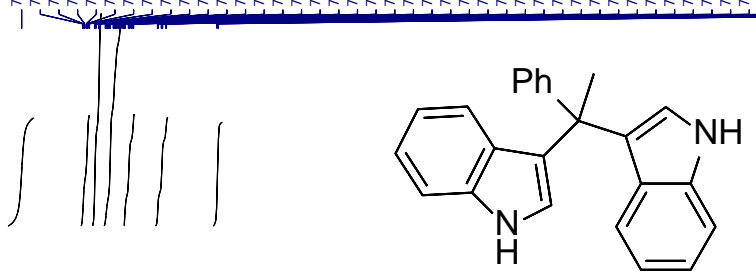

3b

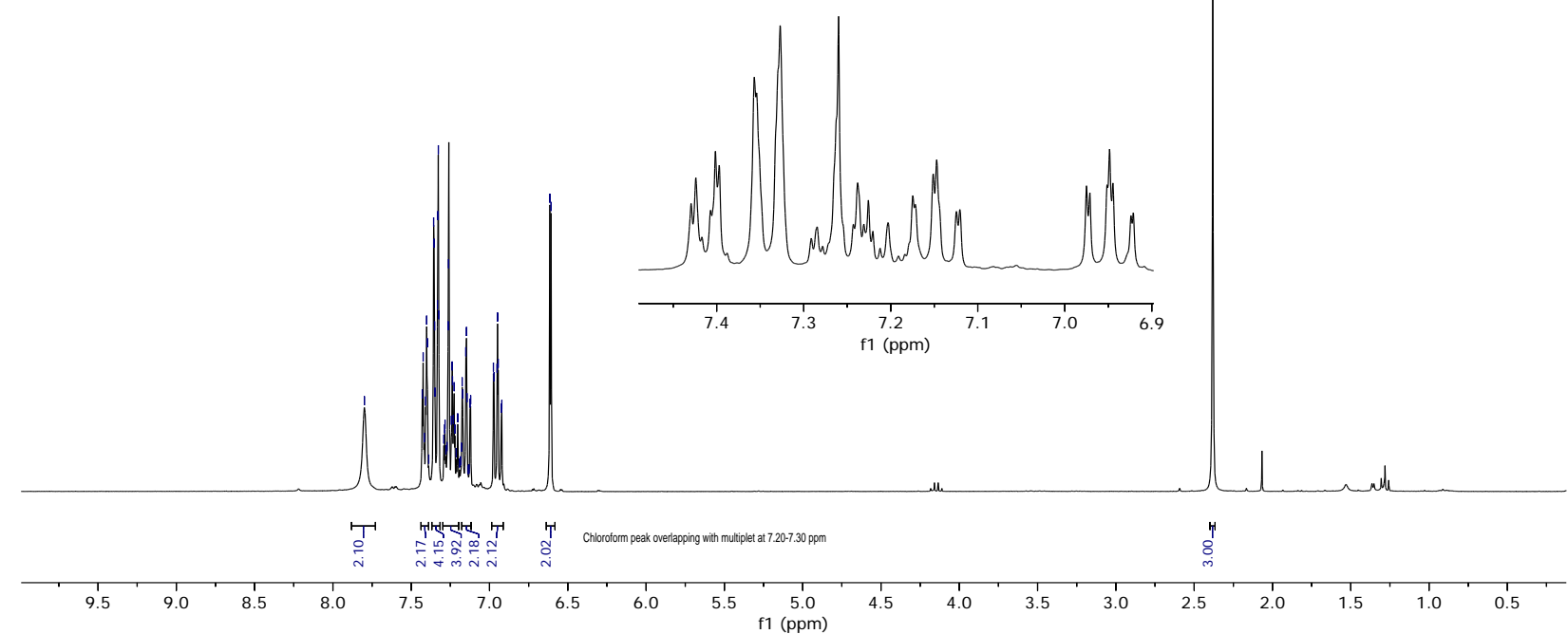

ebmc314char.1.fid

13C 75.5MHz Job 87581 McLean Euan B 314CHAR CDCl3 $25.0 \diamond C \quad 3$ hours 1 min carbon characterisation

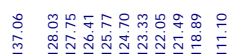
|<smiles>CC(c1ccccc1)(c1c[nH]c2ccccc12)c1c[nH]c2ccccc12</smiles>

3b

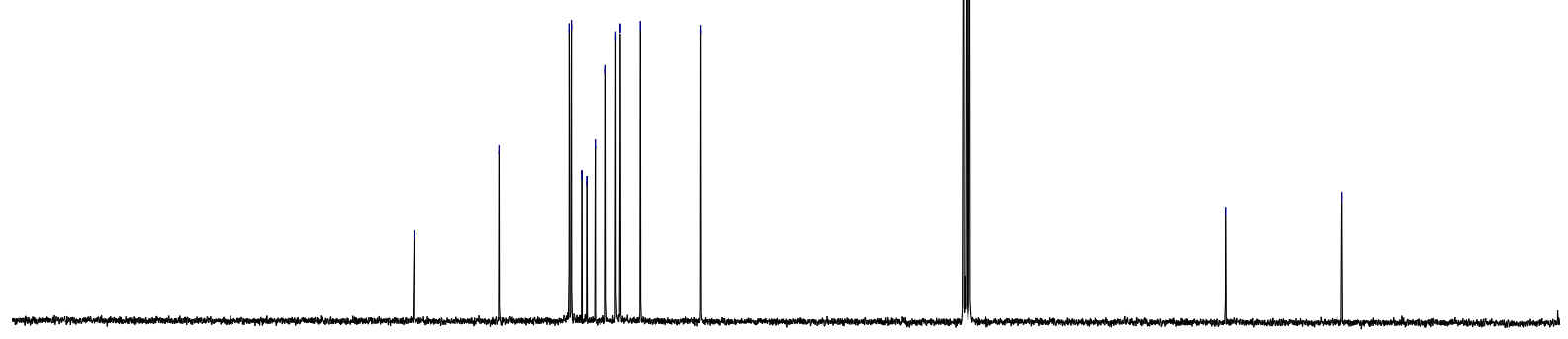

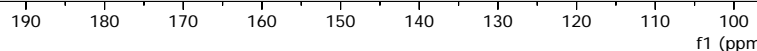


ebmh490char.1.fid

1H 400.1MHz Job 41590 McLean Euan B 490CHAR CDCl3 $24.9 \leadsto C$

proton characterisation
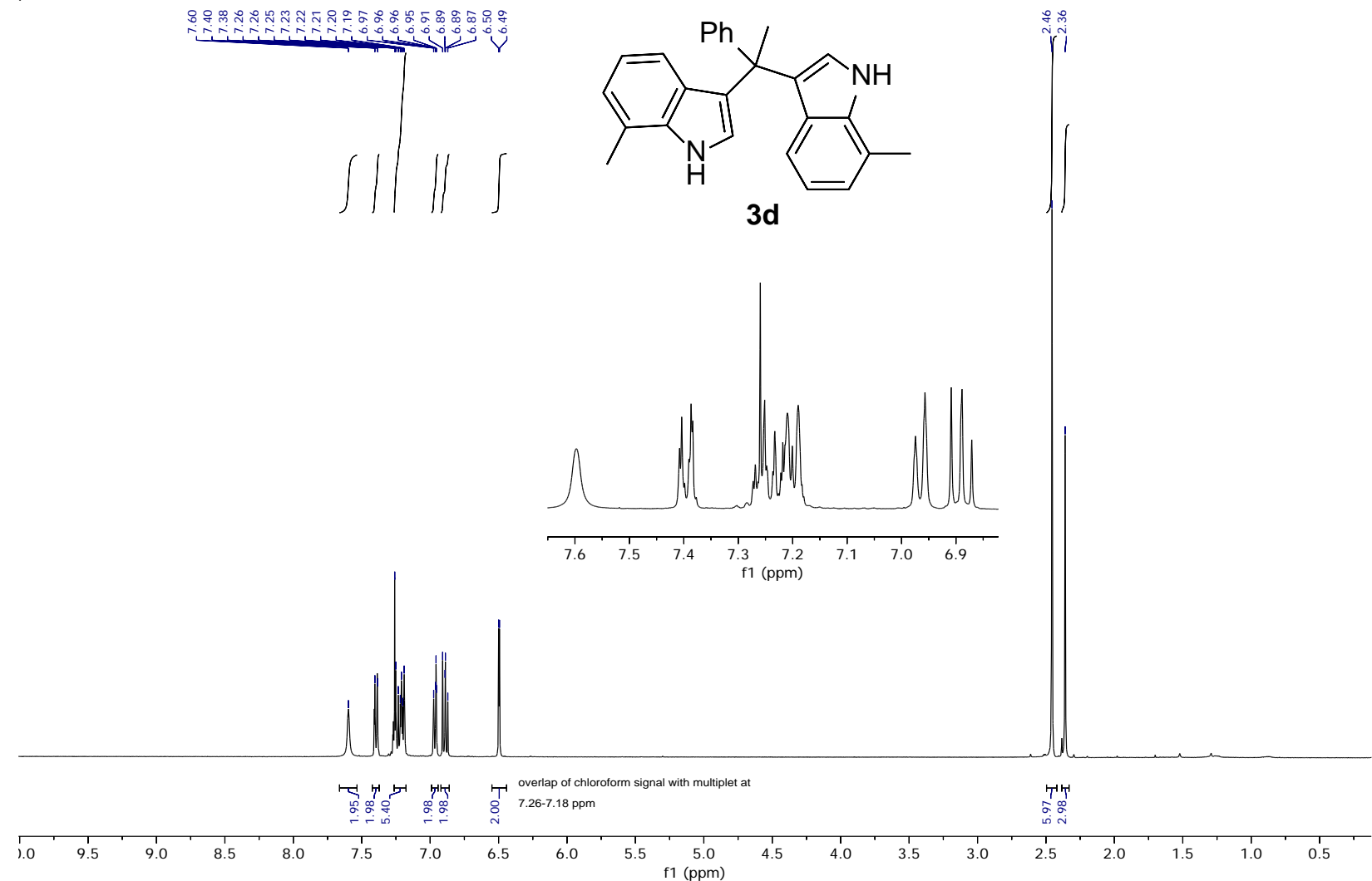

ebmc490char.1.fid

94118 McLean Euan B 490CHAR CDCl3 $25.0 \diamond \mathrm{C} \quad 0$ hour $18 \mathrm{~min}$ carbon characterisation

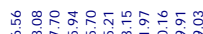

|<smiles>Cc1cccc2c(C(C)(c3ccccc3)c3c[nH]c4c(C)cccc34)c[nH]c12</smiles>

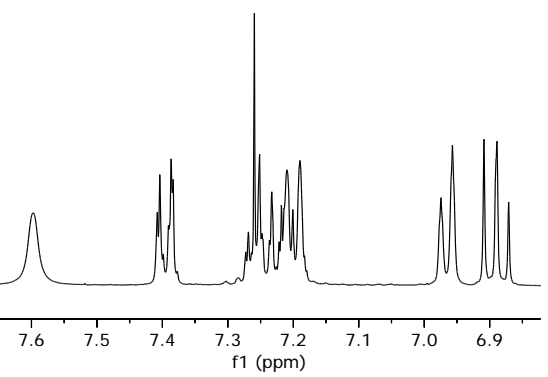


ebmh394char.1.fid

1H 400.1MHz Job 41524 McLean Euan B 394CHAR CDCl3 $24.8 \diamond C$

product characterisation

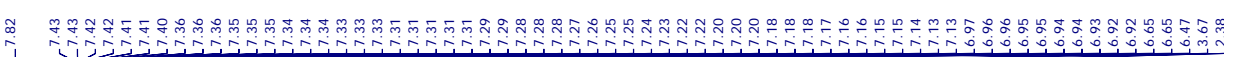

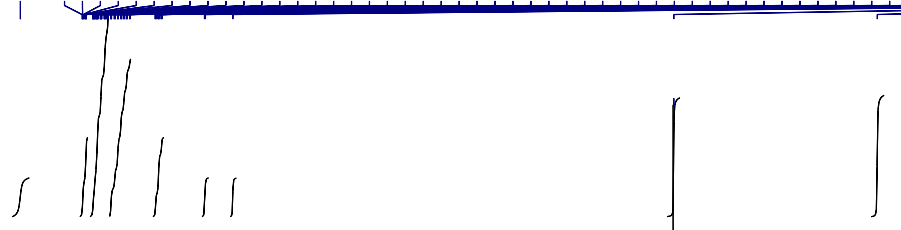<smiles>Cn1cc(C(C)(c2ccccc2)c2c[nH]c3ccccc23)c2ccccc21</smiles>

$5 \mathbf{a}$

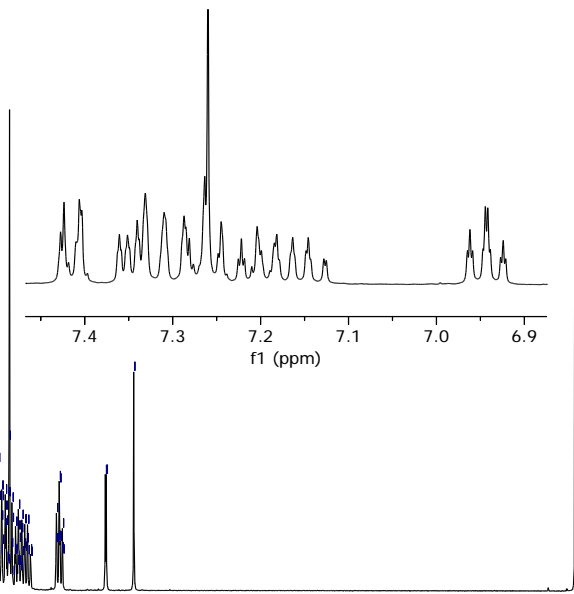

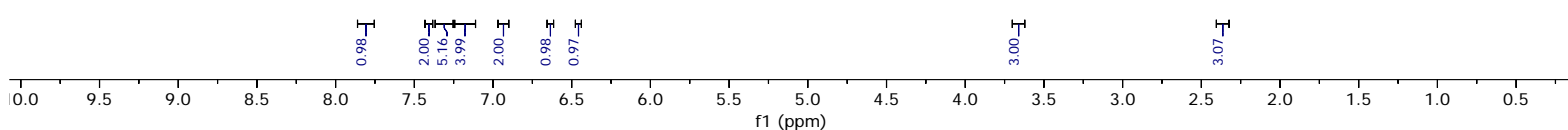

ebmc394cchar.1.fid

13C 100.6MHz Job 41532 McLean Euan B 394CCHAR CDCl3 $26.7 \diamond \mathrm{C} \quad 2$ hours $25 \mathrm{~min}$ carbon characterisation

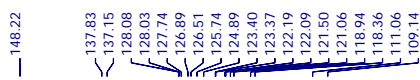

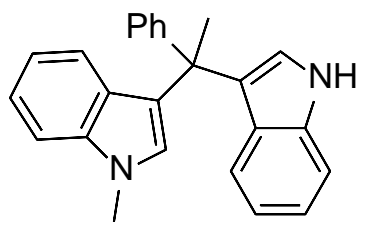

$5 a$

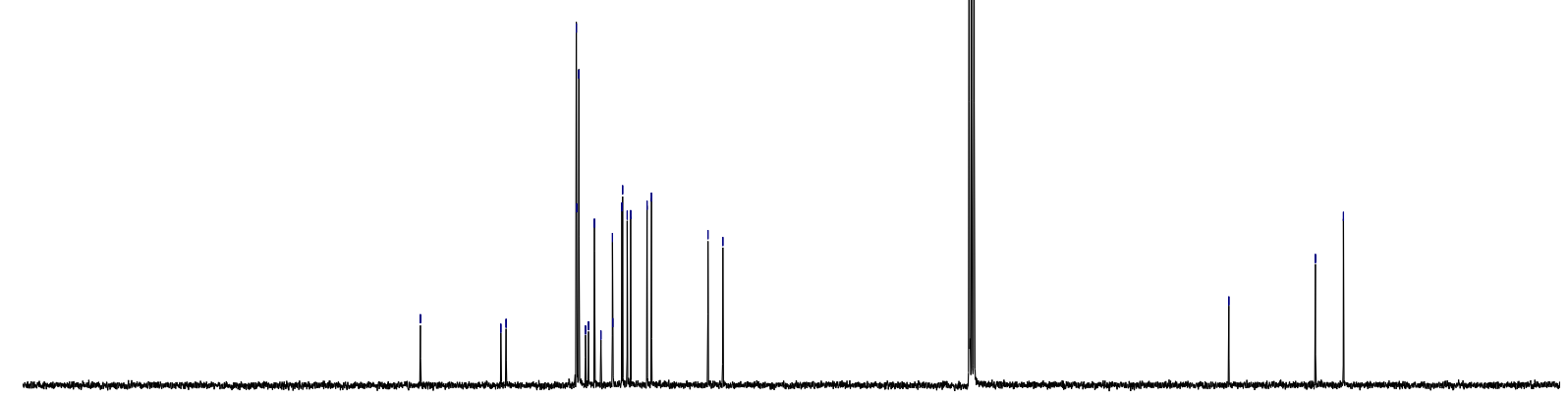

190 


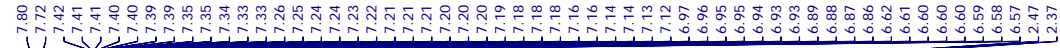

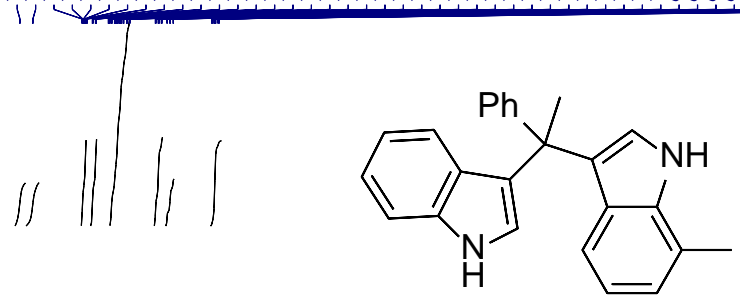

$5 b$

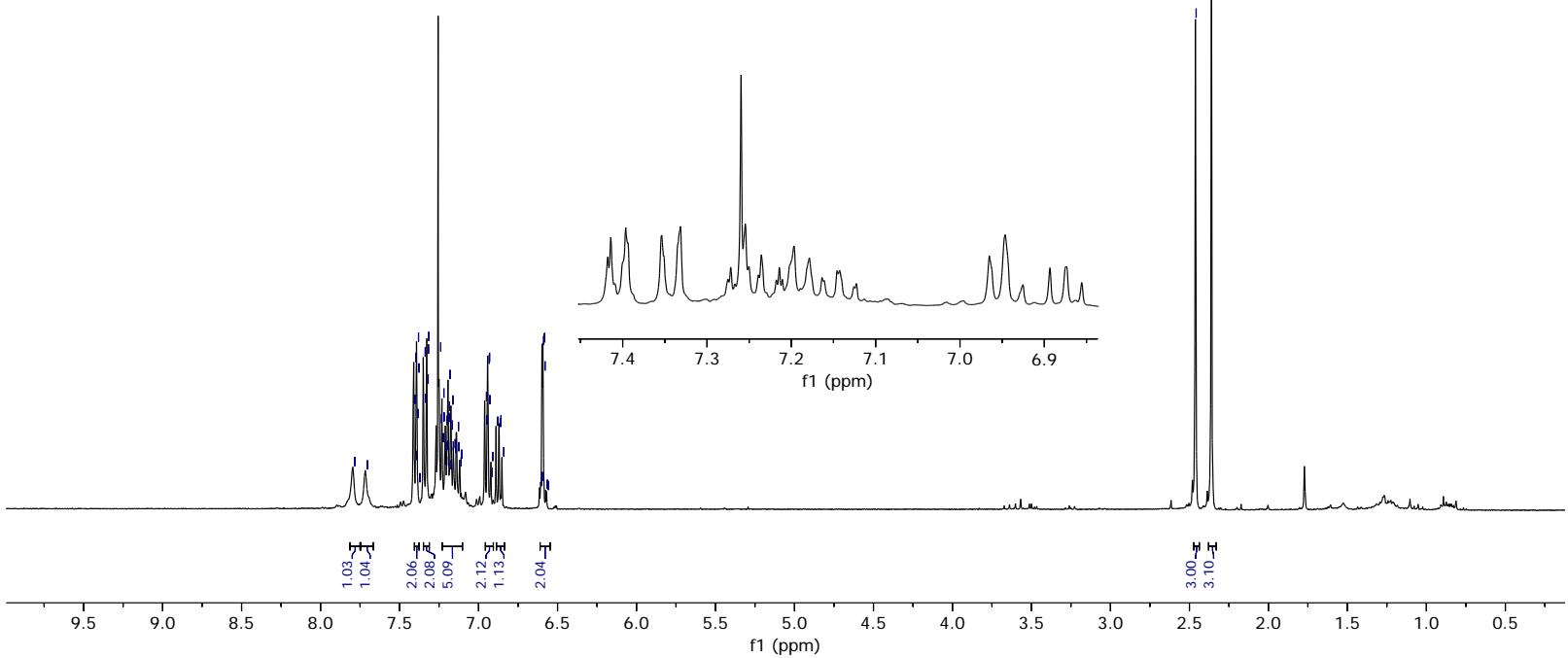

ebmc470bchar.1.fid

13C 100.6MHz Job 41505 McLean Euan B 470BCHAR CDCl3 $26.50 \mathrm{C} \quad 5$ hours 6 min carbon characterisation

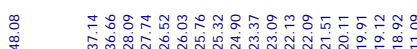

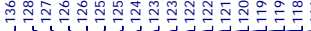

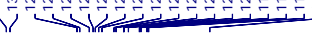

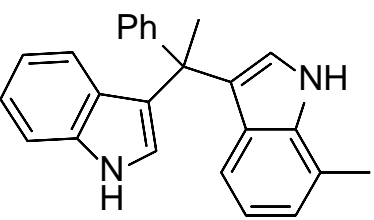

$5 b$

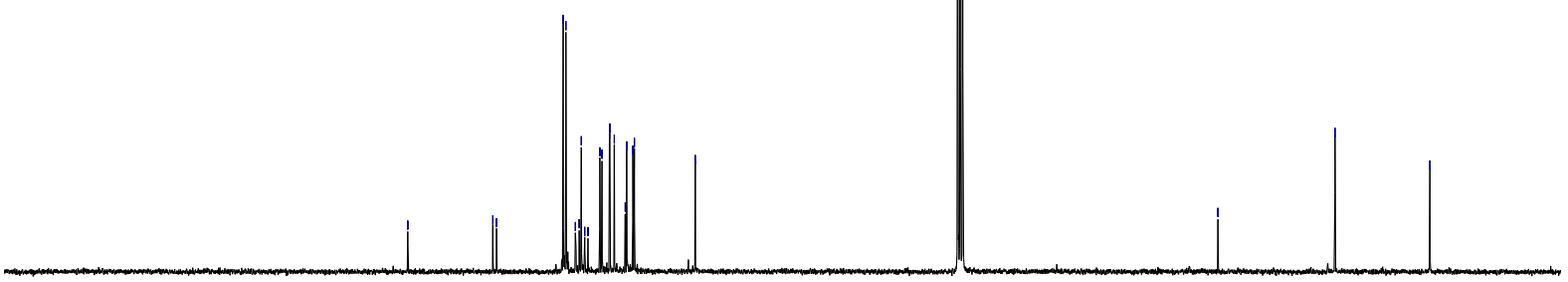

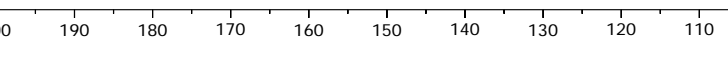

100
$\mathrm{f} 1(\mathrm{ppm})$


ebmh506char2.1.fid

1H 400.1MHz Job 41525 McLean Euan B 506CHAR2 CDCl3 $24.9 \diamond \mathrm{C}$

proton characterisation

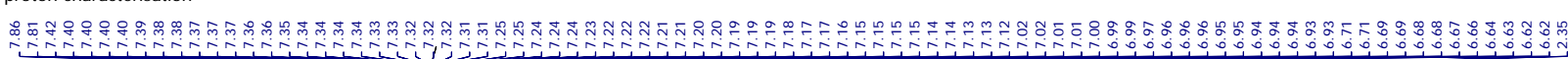

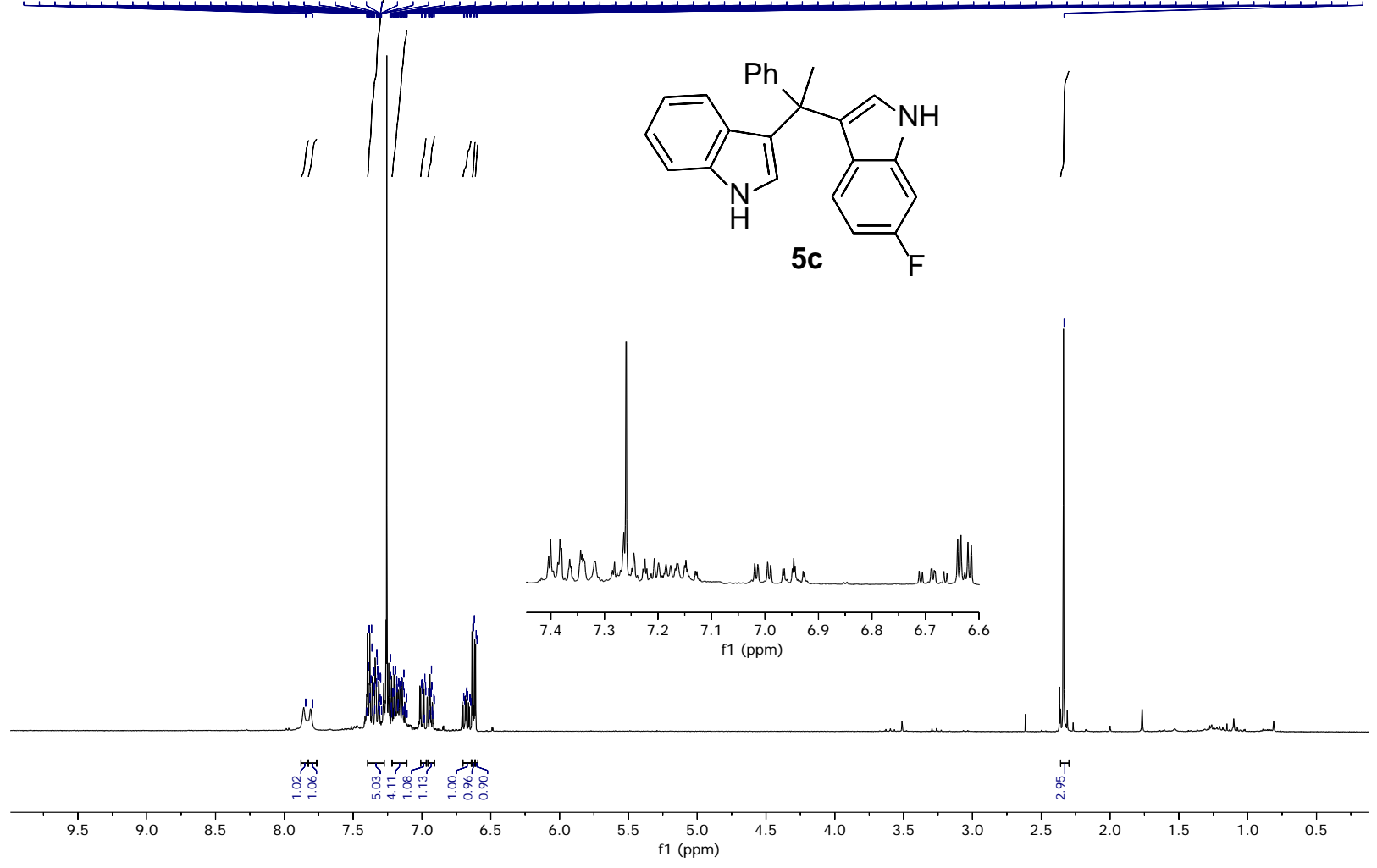

ebmc506char2.1.fid

ebmc506char2.1.fid
$13 \mathrm{C} 100.6 \mathrm{MHz}$ Job 41533 McLean Euan B 506CHAR2 CDC13 $26.7 \diamond \mathrm{C} \quad 4$ hours $37 \mathrm{~min}$
carbon characterisation

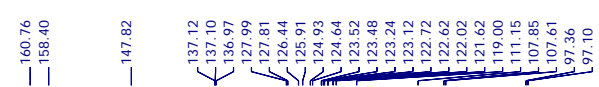

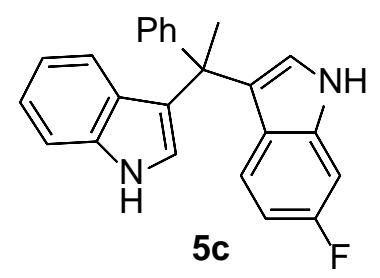

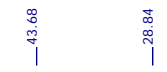
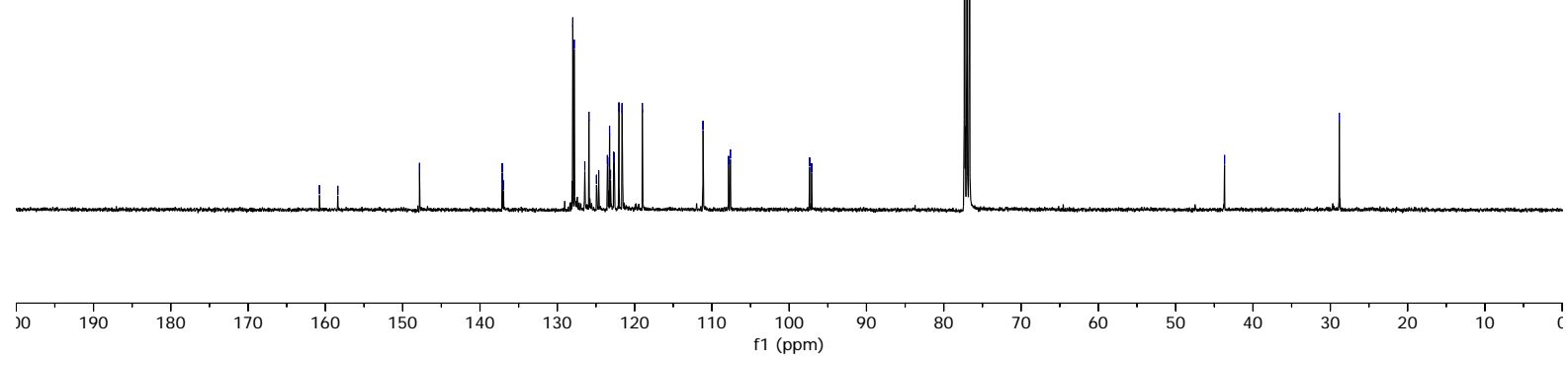

S-55 
ebmf506char2.1.fid

19F 376.5MHz Job 41526 McLean Euan B 506CHAR2 CDCl3 $24.9 \diamond \mathrm{C}$ ohours $1 \mathrm{~min}$ fluorine characterisation

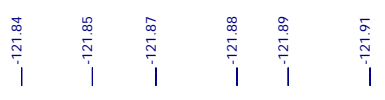

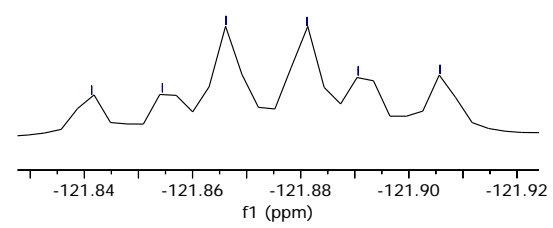

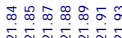

ㄱำ

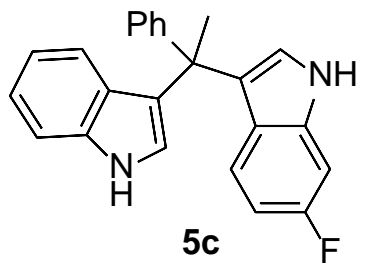

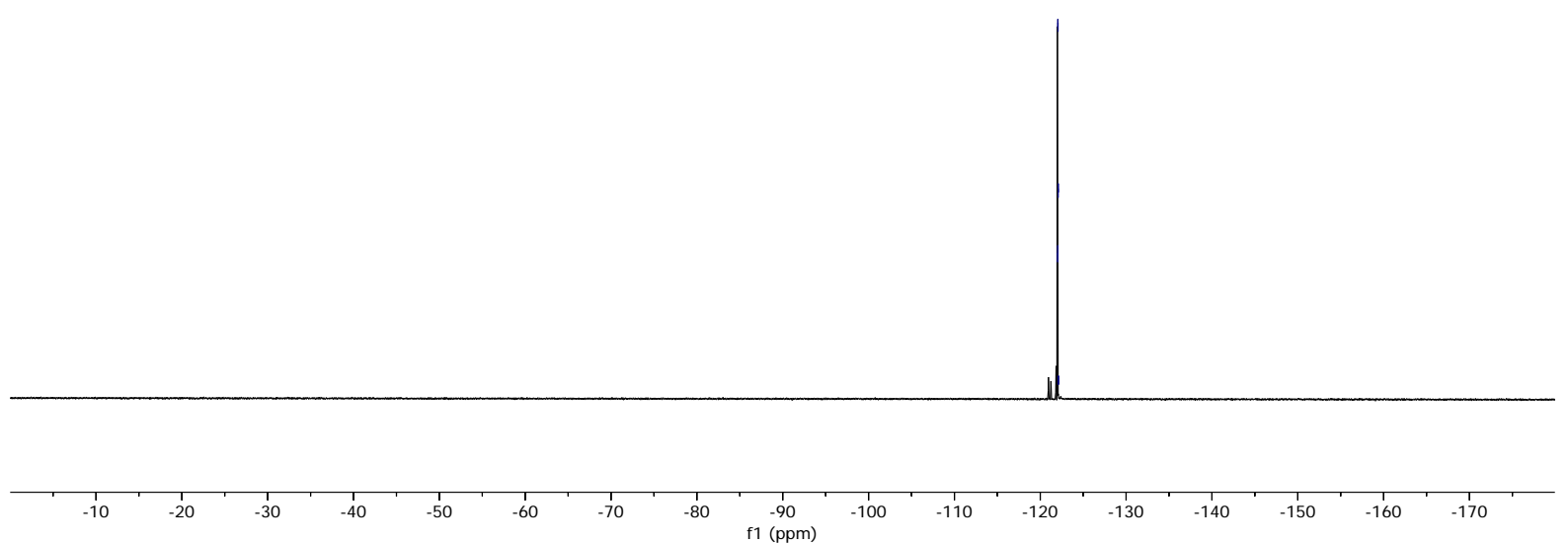


1H 300.1MHz Job 94151 McLean Euan B 507CHAR2 CDCl3 25.1 C

proton characterisation

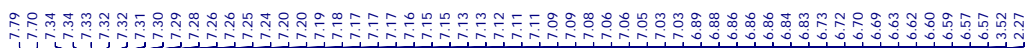
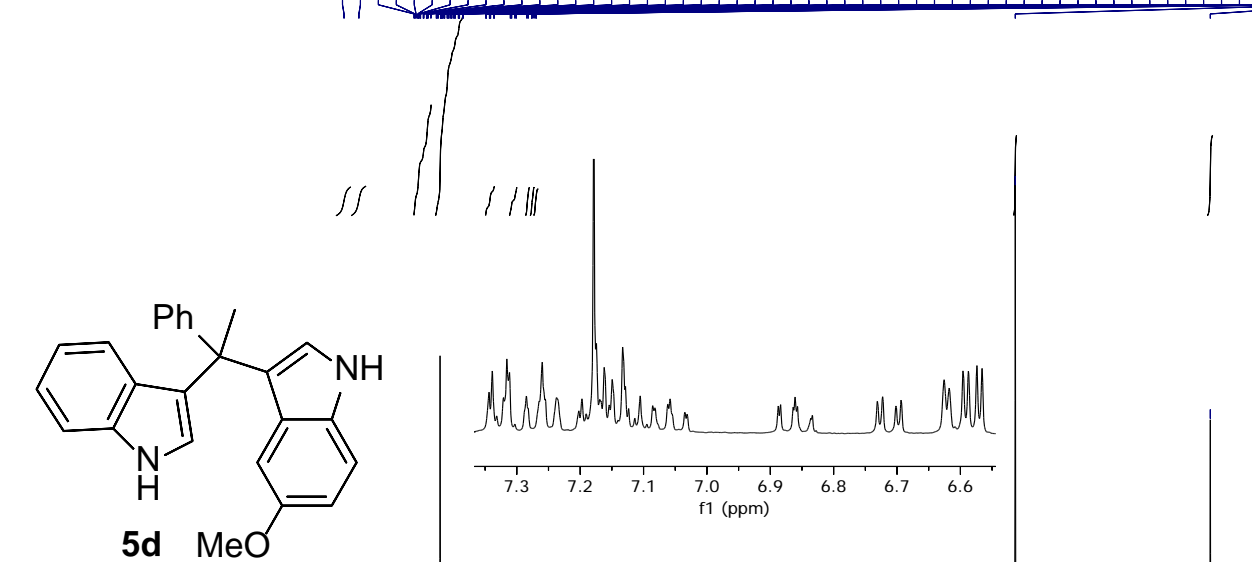

Nhow whona

the Myll

$\begin{array}{llllllll}7.3 & 7.2 & 7.1 & \begin{array}{l}1.0 \\ \text { f1 (ppm) }\end{array} & 6.9 & 6.8 & 6.7 & 6.6\end{array}$

$\mathrm{MeO}$

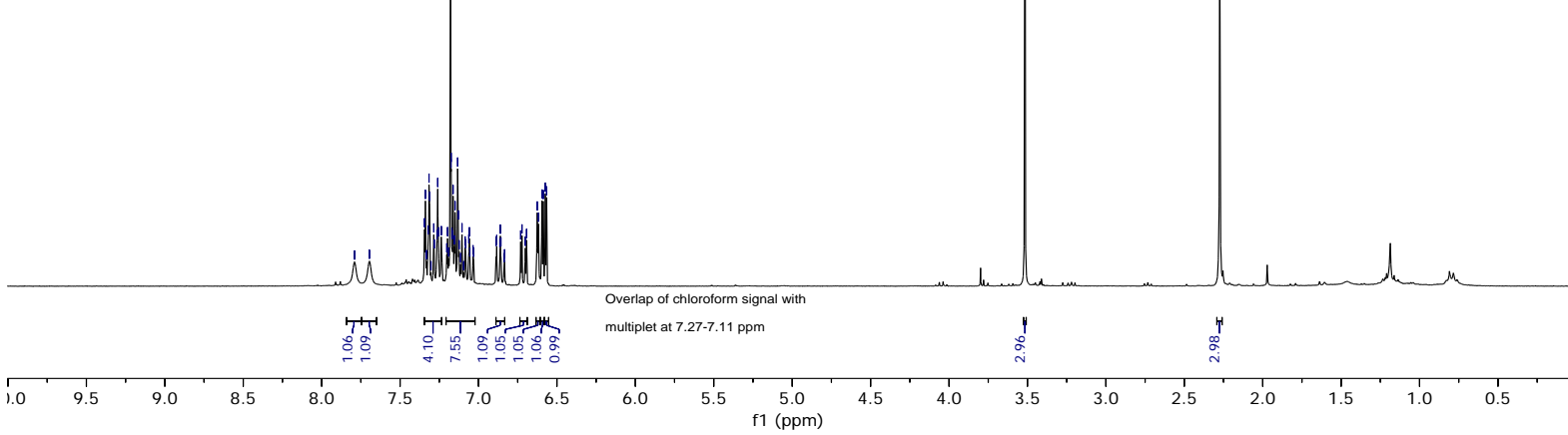

ebmc507char2.1.fid

13C 75.5MHz Job 94161 McLean Euan B 507CHAR2 CDCl3 $25.1 \diamond \mathrm{C} \quad 3$ hours $1 \mathrm{~min}$

carbon characterisation

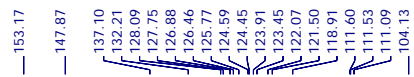
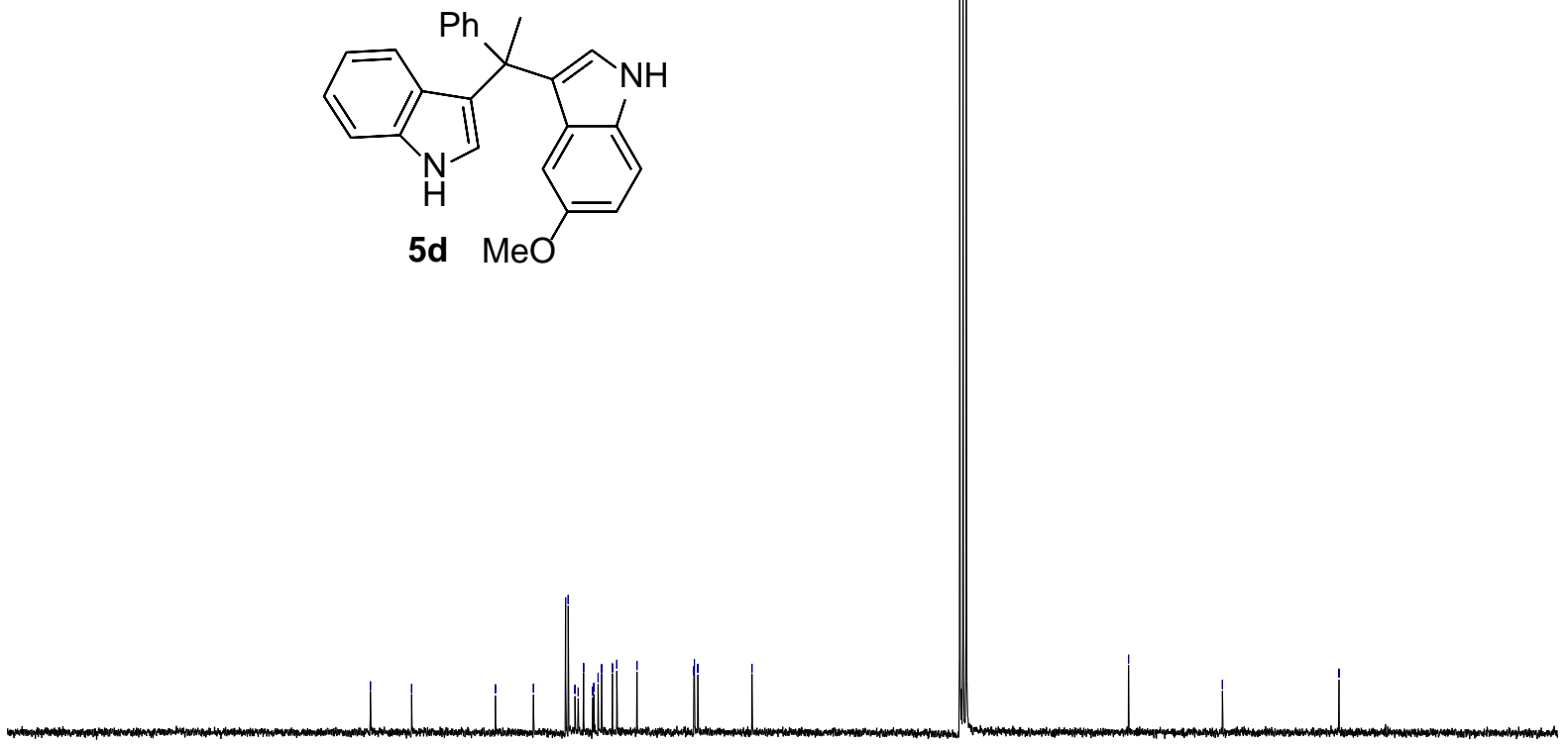

190

$180 \quad 170$

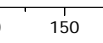

130

110

100
f1 (ppm) 
ebmh515char2.1.fid

1H 300.1MHz Job 94152 McLean Euan B 515CHAR2 CDCl3 25.1队C

proton characterisation

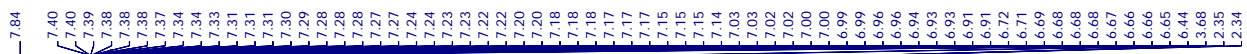

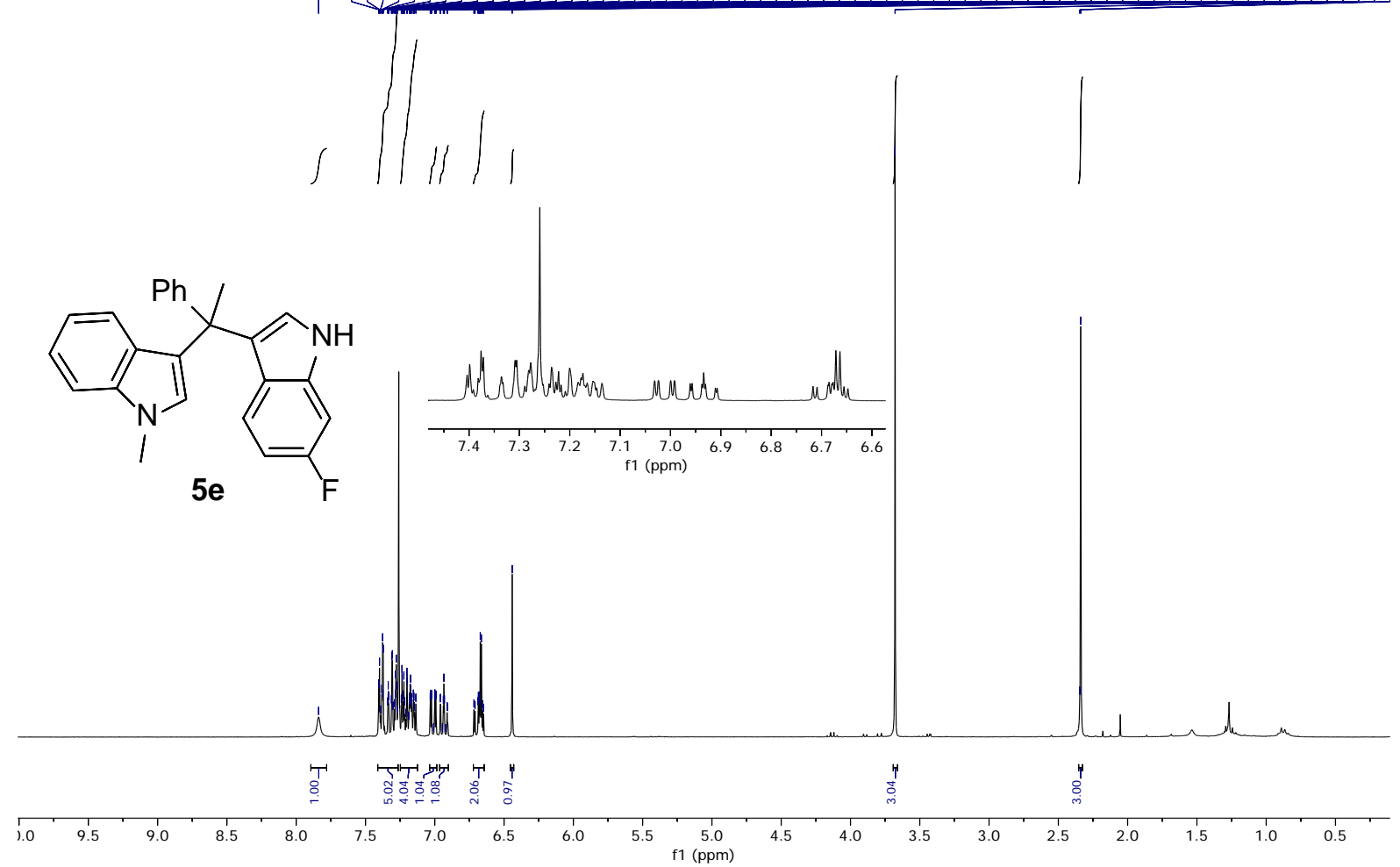

ebmc515char2.1.fid

13C 75.5MHz Job 94162 McLean Euan B 515CHAR2 CDCl3 $25.0 \circlearrowright \quad 3$ hours 1 min carbon character

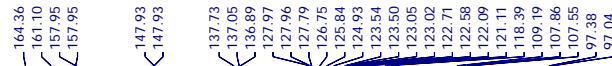
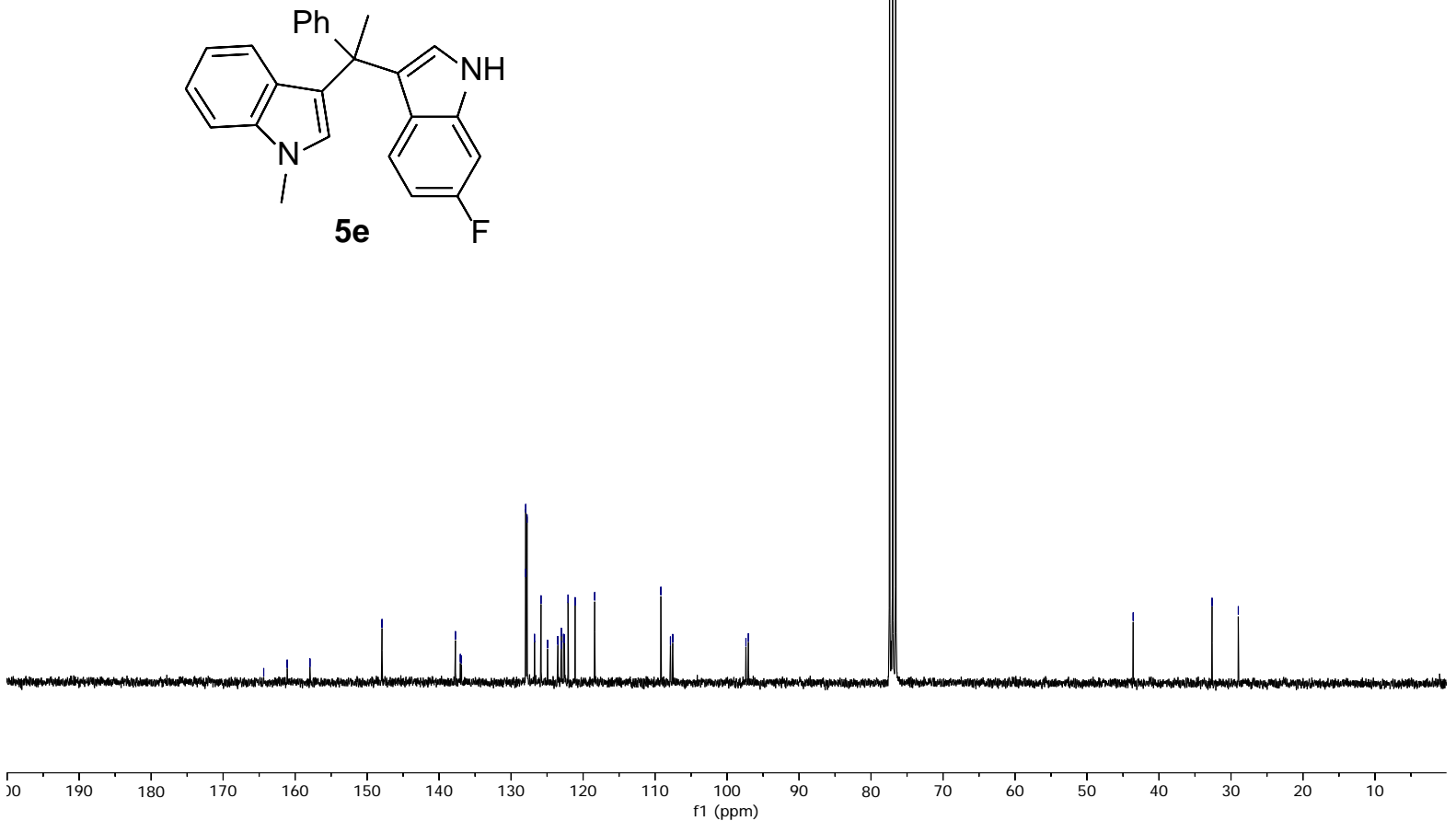

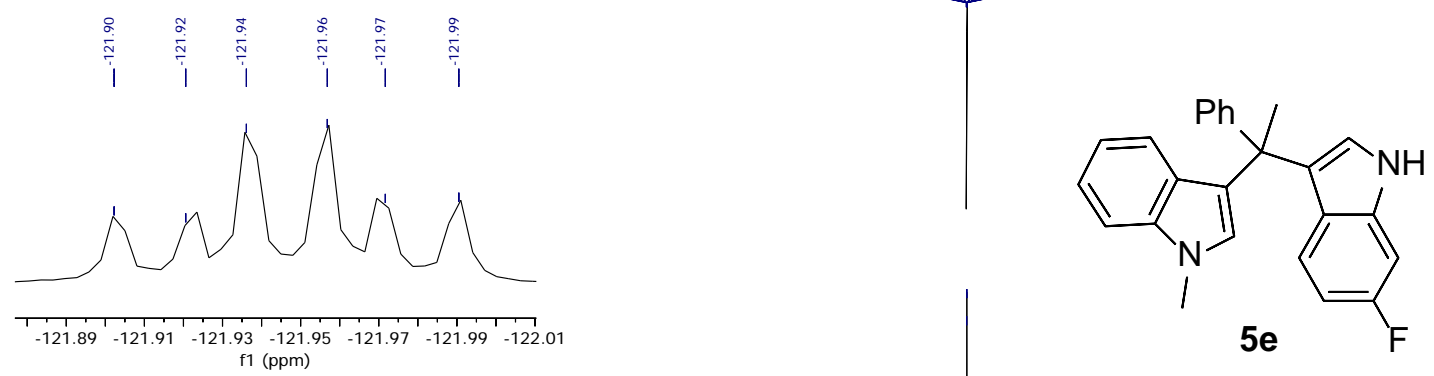

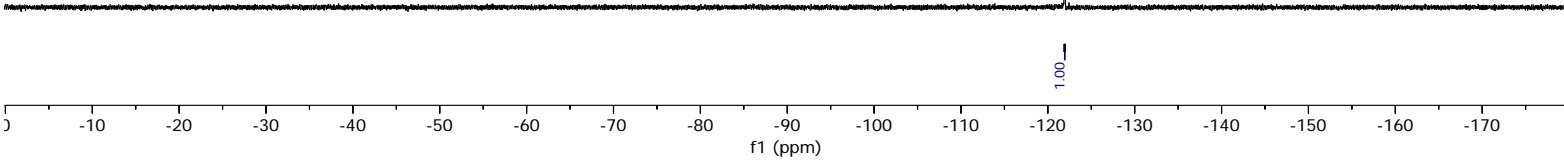

\section{References}

1. $\quad$ Fridkin, G.; Boutard, N.; Lubell, W. D., J. Org. Chem. 2009, 74, 5603-5606.

2. $\quad$ Fredrich, S.; Bonasera, A.; Valderrey, V.; Hecht, S., J. Am. Chem. Soc. 2018, 140, 6432.

3. Dhanak, D.; Reese, C. B., J. Chem. Soc., Perkin Trans. 1986, 2181.

4. Hansen, C. L.; Ohm, R. G.; Olsen, L. B.; Ascic, E.; Tanner, D.; Nielsen, T. E., Org. Lett. 2016, 18, 5990.

5. Taheri, A.; Liu, C.; Lai, B.; Cheng, C.; Pan, X.; Gu, Y., Green Chem. 2014, 16, 3715.

6. Tsuchimoto, T.; Kanbara, M., Org. Lett. 2011, 13, 912-915.

7. Tsuchimoto, T.; Matsubayashi, H.; Kaneko, M.; Nagase, Y.; Miyamura, T.; Shirakawa, E., J. Am. Chem. Soc. 2008, 130, 15823.

8. Kano, S.; Sugino, E.; Shibuya, S.; Hibino, S., J. Org. Chem. 1981, 46, 2979.

9. Ling, F.; Xiao, L.; Fang, L.; Feng, C.; Xie, Z.; Lv, Y.; Zhong, W., Org. Biomol. Chem. 2018, 16, 9274.

10. Zhao, X.; Yu, Z.; Xu, T.; Wu, P.; Yu, H., Org. Lett. 2007, 9, 5263.

11. Bandgar, B. P.; Bettigeri, S. V.; Joshi, N. S., Monatsh. Chem. 2004, 135, 1265.

12. Zhang, Y.; Zhang, S.-X.; Fu, L.-N.; Guo, Q.-X., ChemCatChem 2017, 9, 3107.

13. Guan, X.-K.; Zhang, H.; Gao, J.-G.; Sun, D.-Y.; Qin, X.-S.; Jiang, G.-F.; Zhang, G.-L.; Zhang, S., J. Org. Chem. 2019, 84, 12562. 